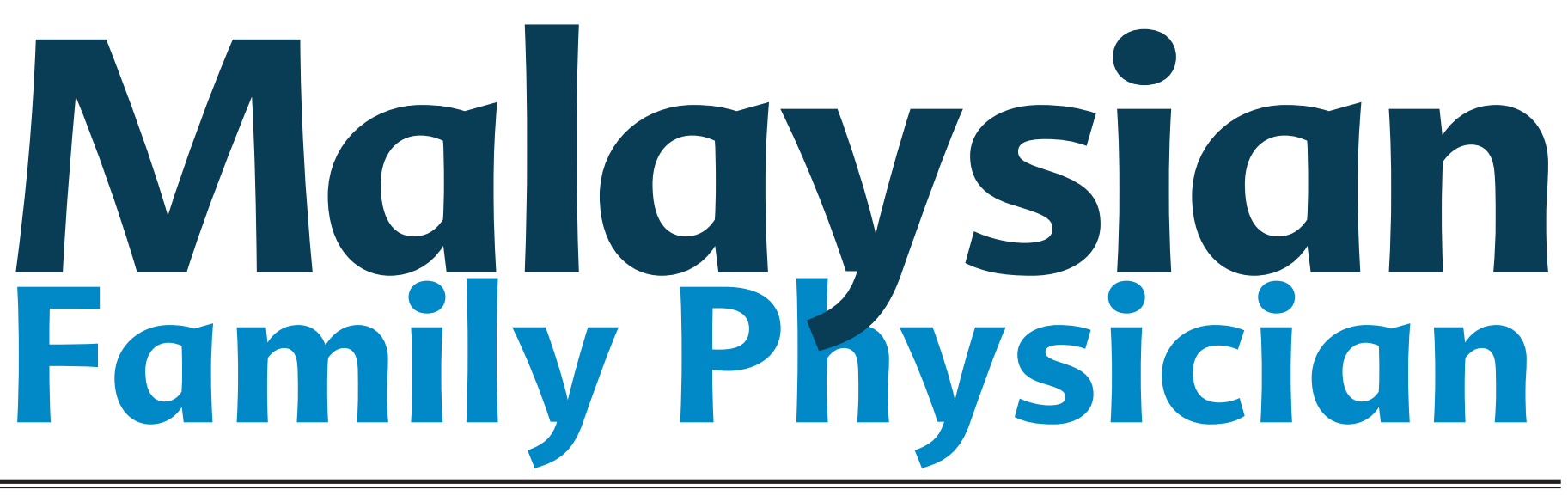

Official Journal of the Academy of Family Physicians of Malaysia and Family Medicine Specialist Association of Malaysia

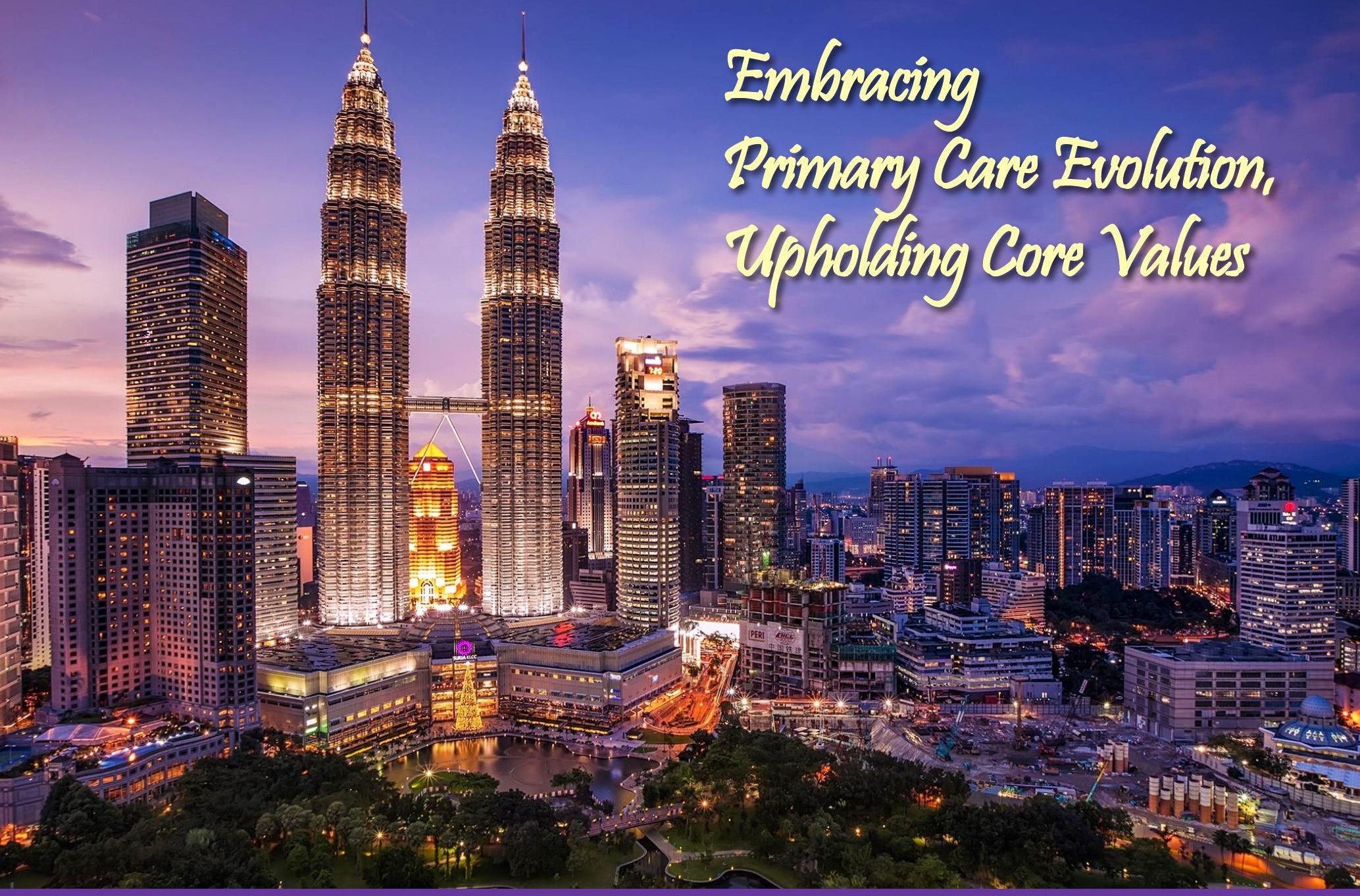

\title{
23RD FAMILY MEDICINE SCIENTIFIC CONFERENCE
}

22rd - 25th September 2021 - Kuala Lumpur

2021 Volume 16 Supplement 1

13.

(5) ISSN

1985-207X (Print)

gigh

1985-2274 (Electronic) 


\section{About MFP}

The Malaysian Family Physician (MFP) is the official journal of the Academy of Family Physicians of Malaysia (AFPM). It is jointly published by the Family Medicine Specialist Association (FMSA) of Malaysia. The MFP is published three times a year. It also started an Online First section in January 2021, where accepted articles are published online ahead of the issue.

Goal: The MFP is an international journal that disseminates quality knowledge and clinical evidence relevant to primary care. The journal acts as the voice of family physicians, researchers and other members of the primary care team on clinical practice issues.

Scope: The MFP publishes:

i. Research - Original Articles and Reviews

ii. Education - Case Reports/Clinical Practice Guidelines/Test Your Knowledge. We only encourage case reports that have the following features:

1. Novel aspects

2. Important learning points

3. Relevant to family practice

iii. Invited debate, commentary, discussion, letters, online, comment, and editorial on topics relevant to primary care.

iv. A Moment in the Life of a Family Physician - We encourage submission of a short narrative to share perspectives, voice, views and opinions about a family physician's experience that has affected their practice or life. This moment should be a reflective piece of fewer than 500 words in length.

Strength: MFP is the only primary care research journal in Malaysia and one of very few in the region. It is open access and fully online. The journal is indexed in Scopus and has a relatively fast review time. The journal has a strong editorial team and an established pool of readers with increasing recognition both locally and internationally.

Circulation: The journal is freely available online.

\section{All correspondence should be addressed to:}

\section{Professor Dr. Su May Liew}

The Editor

The Malaysian Family Physician Journal

Academy of Family Physicians of Malaysia,

Unit 1-5, Level 1 Enterprise 3B Technology Park Malaysia (TPM)

Jalan Innovasi 1 Lebuhraya Puchong-Sungai Besi

Bukit Jalil, 57000 Kuala Lumpur.

Tel: +603-89939176/9177

Fax: $603-89939187$

Email: journal.mfp@gmail.com

Website: http://www.e-mfp.org

\section{Publication Ethics}

Ethics

Evidence of ethics approval and informed consent should be included in the manuscript for studies involving animal experiments or human participants.

Competing interests

MFP requires authors to declare all conflicts of interest in relation to their work. All submitted manuscripts must include a 'competing interests' section at the end of the manuscript (before references) listing all competing interests.

\section{Ethical Guidelines for Authors}

Authorship credit should be based only on:

1. Substantial contributions to conception and design, or acquisition of data, or analysis and interpretation of data;

2. Drafting the article or revising it critically for important intellectual content; and

3. Final approval of the version to be published.

4. Agreement to be accountable for all aspects of the work ensuring that questions related to the accuracy or integrity of any part of the work are appropriately investigated and resolved.

\section{Plagiarism Policy}

The journal takes a serious view on cases of plagiarism and research misconduct. All submitted articles are checked for plagiarism. If plagiarism or research misconduct is suspected, a thorough investigation will be carried out and action taken according to COPE guidance found at https://publicationethics.org/resources/flowcharts.

Open Access Policy

Upon acceptance, all articles in the Malaysian Family Physician are immediately and permanently free for everyone to read and download.

Disclaimer: Although an official publication, the Malaysian Family Physician provides a forum for free expression and exchange of views among those in the profession. Therefore, views expressed in published articles are not necessarily those of the Journal, AFPM or FMSA. The views of the editor need not reflect the views of the Academy. No portion of any matter appearing in the Malaysian Family Physician may be quoted or republished in any form without the prior written consent of the author, editor and the AFPM. 


\section{Editorial Board}

\section{Chief Editor}

Professor Dr Su-May Liew (su_mayliew@um.edu.my)

\section{Deputy Chief Editor}

Professor Dr Ping Yein Lee (pylee02@gmail.com)

\section{Associate Editors}

Associate Professor Dr Adina Binti Abdullah (adinabdullah@um.edu.my)

Associate Professor Dr Ai Theng Cheong (caitheng@gmail.com)

Associate Professor Dr Boon How Chew (chewboonhow@gmail.com)

Professor Dr Ee Ming Khoo (khooem@um.edu.my)

Professor Dr Harmy Bin Mohamed Yusoff (harmyusoff@unisza.edu.my)

Dr Irmi Zarina Binti Ismail (irmiismail@upm.edu.my)

Associate Professor Dr Nik Sherina Haidi Binti Hanafi (sherina@ummc.edu.my)

Dr Ping Foo Wong (pingfoo@hotmail.com)

Dr Say Hien Keah (richardkeah8282@gmail.com)

Professor Dr Sazlina Binti Shariff Ghazali (drsazsg@gmail.com)

Associate Professor Dr Siew Mooi Ching (sm_ching@upm.edu.my)

Dr Sylvia McCarthy (sylvia@hospismalaysia.org)

Dr V Paranthaman P Vengadasalam (drparan@gmail.com)

Dr Wai Khew Lee (leewaikhew@hotmail.com)

Dr Zainal Fitri Bin Zakaria (drzainalfitri@moh.gov.my)

\section{Local Advisors}

Professor Dr Chirk Jenn Ng (ngcj@um.edu.my)

Professor Datin Dr Yook Chin Chia (chiayc@um.edu.my)

Professor Dr Wah Yun Low (lowwy@um.edu.my)

Associate Professor Datuk Dr DM Thuraiappah (dmthuraiappah@gmail.com)

\section{International Advisors}

Professor Dr Cindy Lo-Kuen Lam (Hong Kong)

Professor Dr John W Beasle (USA)

Professor Dr Julia Blitz (South Africa)

Associate Professor Dr Lee Gan Goh (Singapore)

Professor Dr Michael Kidd (Australia)

Professor Dr Moyez Jiwa (Australia)

Professor Dr Nigel J Mathers (United Kingdom) 


\section{Information for Authors}

The Malaysian Family Physician welcomes articles on all aspects of family medicine in the form of original research papers, review articles, CPG review, case reports, test your knowledge and letters to the editor. The journal also publishes invited debate, commentary, discussion, letters, comment, and editorials on topics relevant to primary care.

Articles are accepted for publication on condition that they are contributed solely to the Malaysian Family Physician. Neither the Editorial Board nor the Publisher accepts responsibility for the views and statements of authors expressed in their contributions. All papers will be subjected to peer review. The Editorial Board further reserves the right to edit and reject papers. Authors are advised to adhere closely to the instructions given below to avoid delays in publication.

All manuscripts must be submitted through the Open Journal System (OJS) at http://e-mfp.org/ojs

\section{SUBMISSION REQUIREMENTS}

1. The author must declare that the manuscript has not been previously published, nor is it being considered for publication in another journal concurrently.

2. The Main Manuscript should be submitted in electronic form only and in Microsoft Word.

- The manuscript contains all the sub-headings required for the article type (refer below).

- The manuscript uses a single-spaced, 12-point font and uses italics rather than underlining (except URL addresses).

- All figures, tables and illustrations are placed at the appropriate sections in the manuscript file rather than at the end of the manuscript or submitted separately.

- Use left-aligned paragraph formatting rather than full justification.

- Follow the instructions in Ensuring a Blind Review (refer below).

- Follow the referencing style provided in the References section below.

- Provide URLs for references where available.

- Where available, URLs for the references have been provided.

3. The Title Page must be uploaded separately from the main manuscript file in Microsoft Word. Please refer to the required sub-headings in the Title Page section below.

4. A Cover Letter must be signed by the corresponding author on behalf of all authors. This letter must include this statement "this manuscript is my (our) own work, it is not under consideration by another journal, and this material has not been previously published."

5. All authors must sign the Declaration Form and submit it together with the manuscript and cover letter. Please download the form here.

6. Please enter all authors' name and email address in the submission portal.

7. When preparation your manuscript, please follow the Uniform Requirements for Manuscripts Submitted to Biomedical Journals recommended by the International Committee of Medical Journal Editors (http://www.icmje.org/icmje-recommendations.pdf).

\section{TITLE PAGE}

For all types of manuscript, please include all the sub-headings below in the Title Page:

- Article Type: Original Research / Review / CPG Review / Case Report / Test Your Knowledge / Letter To Editor

- Title: Please state the title in detail to include the study design, particularly for original research.

- Author(s): The full names, professional qualifications (limited to two only) and institutions of all authors.

- Shortened name of author(s): should be written in the style of surname or preferred name followed by initials, e.g. Abdullah KS, Rajakumar MK, Tan WJ, for future indexing.

- Corresponding Author: Corresponding author's mailing address, designation, institution and contact details (email, telephone and fax numbers)

- Funding: Please state if the study was funded; if so, by which institution and the funding ID.

- Ethical Approval: Please state if the study was approved; if so, by which institution and the approval ID.

- Conflicts of interest: Please state if any author has a conflict of interest.

\section{MAIN MANUSCRIPT}

For every article submitted, please follow the requirements according to the type of article.

\section{Original Research (Including Clinical Audit Article)}

The original research (including clinical audit) should be conducted in the primary care setting on a topic of relevance to family practice. Both qualitative and quantitative studies are welcome. The length should not exceed 3000 words with a maximum of 5 tables or figures and 30 references. Please include the following sub-headings in the manuscript:

1. Title: State the title based on PICO, including study design.

2. Abstract: Structured abstract (Introduction, Methods, Results and Conclusion) of no more than 250 words.

3. Keywords: 3-5 keywords, preferably MeSH terms.

4. Introduction: Clearly state the purpose of the article with strictly pertinent references. Do not review the subject extensively.

5. Methods: Describe the study in sufficient detail to allow others to replicate the results. Provide references to established methods, including statistical methods; provide references and brief descriptions of methods that have been published but are not well known; describe new or substantially modified methods, give reasons for using them, and evaluate their limitations. When mentioning drugs, generic names are preferred (proprietary names can be provided in brackets). Do not use patients' names or hospital numbers. Include numbers of observation and the statistical significance of the findings. When appropriate, state clearly that the research project has received the approval of the relevant ethical committee. For an RCT article, please include the trial registration number) and follow the CONSORT checklist. Other study designs must also follow a reporting checklist, which can be found at https://www.equator-network.org/.

6. Results: Present your results in logical sequence in the text, tables and figures. Tables and figures may be left at the respective location within the text. These should be numbered using Arabic numerals only. Table style should be "Simple" (as in Microsoft Word). Do not repeat table or figure data in the text.

7. Discussion: Emphasise the new and important aspects of the study and conclusions that follow from them. Do not repeat data given in the Results section. The discussion should state the implications of the findings and their limitations and relate the observations to the other relevant studies. Link the conclusions with the aims of the study but avoid unqualified statements and conclusions not completely supported by your data. Recommendations, when appropriate, may be included.

8. Acknowledgements: Acknowledge grants awarded in aid of the study and people who have contributed significantly to the study (but do not qualify for authorship).

9. Conflicts of interest: All authors must declare any conflicts of interest.

10. How does this paper make a difference in general practice?: This section should be written in bullet points (up to five points) and must not exceed 100 words.

11. References: Refer to the References section below for more details. 
All types of review articles, including narrative review, scoping reviews and systematic reviews are accepted for publication in MFP. A comprehensive review of the literature with a synthesis of practical information for practising doctors is expected. For a systematic review, the PRISMA checklist (https://www.equator-network.org/reporting-guidelines/prisma/) must be followed. For a scoping review, the PRISMA-ScR checklist (https://www.equator-network.org/reporting-guidelines/prisma-scr/) should be followed. The length should not exceed 4000 words with a maximum of 5 tables or figures and 40 references. Please include the following sub-headings in the manuscript:

1. Title: Include the topic and type of review in the title.

2. Abstract: Structured abstract (Introduction, Methods, Results and Conclusion) of no more than 250 words.

3. Keywords: 3-5 keywords, preferably MeSH terms.

4. Introduction: Describe the topic and objective of the review.

5. Methods: All types of review articles (including narrative review) must report the search strategy, database and keywords used to obtain the literature. The PRISMA and PRISMA-ScR checklists should be followed for systematic and scoping reviews, respectively.

6. Results (*for systematic and scoping reviews): This section is required for systematic and scoping reviews. Please follow the guideline in the PRISMA and PRISMA-ScR checklists.

7. Discussion ( ${ }^{*}$ for systematic and scoping reviews): This section is required for systematic and scoping reviews. Please follow the guideline in the PRISMA and PRISMA-ScR checklists.

8. Any relevant subheadings (*for narrative review): A narrative review may have any other relevant sub-headings according to needs.

9. Conclusion: Provide a conclusion by linking to the objective of the review.

10. Acknowledgements: Acknowledge grants awarded in aid of the study and people who have contributed significantly to the study (but do not qualify for authorship).

11. Conflicts of interest: All authors must declare any conflicts of interest.

12. How does this paper make a difference in general practice?: This section should be written in bullet points (up to five points) and must not exceed 100 words.

13. References: Refer to the References section below for more details.

\section{Case Report}

Case reports should preferably be less-commonly seen cases that have an educational value for practising doctors. Only case reports that are novel, have important learning points and relevant to family practice will be accepted for publication in this journal. The case report must be written in a patient-centred manner instead of a disease-centred focus. The length should not exceed 1500 words and cite no more than 20 references. Before submitting the case report, the authors must ensure that the patient's identity is protected both in the text and pictures. Please include the following sub-headings in the manuscript:

1. Title: Use an interesting title to show the new learning points and include the term "case report" in the title.

2. Abstract: Unstructured abstract between 100-250 words.

3. Keywords: 3-5 keywords, preferably MeSH terms.

4. Introduction: Describe the condition and aim of the case report.

5. Case Presentation: Describe the case in detail.

6. Discussion: Discuss the case with existing literature.

7. Conclusion: Provide the key learning point from the case report

8. Acknowledgements: Acknowledge grants awarded in aid of the study and people who have contributed significantly to the study (but do not qualify for authorship).

9. Conflicts of interest: All authors must declare any conflicts of interest.

10. Patients' consent for the use of images and content for publication: Was consent obtained from the patient(s)? Was the consent written or verbal?

11. What is new in this case report compared to the previous literature?: This section should be written in bullet points (up to five points) and must not exceed 100 words.

12. What is the implication to patients?: Describe any potential implication to patients based on the learning points from this case report.

13. References: Refer to the References section below for more details.

\section{CPG Review}

The CPG should be relevant to primary care. Its length should not exceed $\mathbf{4 0 0 0}$ words and $\mathbf{4 0}$ references. An abstract is required (no more than 300 words) together with the keywords. The CPG review should be written with case vignettes to illustrate its application in primary care practice.

1. Title: State the scope of the CPG, include the latest version or year for revised CPGs.

2. Abstract: Unstructured abstract between 100-250 words.

3. Keywords: 3-5 keywords, preferably $\mathrm{MeSH}$ terms.

4. Introduction: Describe the condition and aim of the CPG review.

5. Development process of the CPG: Describe the development process of the CPG, e.g.: who are the team members involved, what methodology was used, how was the evidence gathered, how was the decision made on the recommendations, was the outcomes validated, how was the CPG disseminate and implementation, etc. Follow the AGREE Reporting Checklist (https:/www.equator-network.org/wpcontent/uploads/2016/03/AGREE-Reporting-Checklist.pdf) wherever possible.

6. Key recommendations of the CPG: Describe the key recommendations primary care doctors should know.

7. Key changes in the CPG (only applicable for revised CPGs): Describe the key changes or updates from the previous CPG.

8. How to apply the CPG into practice in primary care? Explain how the CPG can be used in primary care practice.

9. Case vignettes as examples of application: Use case vignettes to illustrate the application of the CPG.

10. Conclusion: Summarise the key learning points.

11. Acknowledgements: Acknowledge grants awarded in aid of the study and people who have contributed significantly to the study (but do not qualify for authorship).

12. Conflicts of interest: All authors must declare any conflicts of interest.

13. How does this paper make a difference in general practice?: This section should be written in bullet points (up to five points) and must not exceed 100 words.

14. References: Refer to the References section below for more details. 


\section{Test Your Knowledge}

A Test Your Knowledge article should be relevant to primary care and preferably be about less-commonly seen cases that have an educational value for practising doctors. The length should not exceed 1000 words and no more than 20 references. Please include the following subheadings in the manuscript:

1. Title: State the title in a question format.

2. Abstract: Unstructured abstract between 100-250 words.

3. Keywords: 3-5 keywords, preferably MeSH terms.

4. Case Summary: Describe the case.

5. Questions: State the questions.

6. Answers with discussion: Provide the answers and discuss them with support from the literature.

7. Acknowledgements: Acknowledge grants awarded in aid of the study and people who have contributed significantly to the study (but do not qualify for authorship).

8. Conflicts of interest: All authors must declare any conflicts of interest.

9. How does this paper make a difference in general practice?: This section should be written in bullet points (up to five points) and must not exceed 100 words.

10. References: Refer to the References section below for more details.

\section{Letter to Editor}

A letter to the editor should be of relevance to primary care and in response to an article or topic published in previous issues of this journal. The length should not exceed 1000 words and cite no more than 20 references. Please include the following sub-headings in the manuscript:

1. Title: State the title clearly.

2. Keywords: 3-5 keywords, preferably MeSH terms.

3. Main text: Start the manuscript with "Dear editor:". There is no specific required heading. Authors can create any sub-headings as necessary.

4. References: Refer to the References section below for more details.

\section{REFERENCES}

Number references consecutively in the order in which they are first mentioned in the text. Identify references in text, tables and legends by Arabic numerals (in superscript). Please use the citation style adopted by the National Library of Medicine, Maryland, USA, (http://www. pubmed.gov), some examples are given below. For indexed journals, the short forms for the journal names can be accessed at the PubMed website (search within Journal Database). Where possible, avoid citing abstracts, personal communication or unpublished data as references. Include among the references manuscripts accepted but not yet published and designate the journal followed by "in press" (in parenthesis). When referencing a website, please include the full title and accessed date. Notice article titles are capitalised in sentence case; book and journal titles are capitalised in title case. Some examples of reference list entries:

- Standard journal article: List up to three authors only; when four or more, list only the first three and add et al. Connor EM, Sperling RS, Gelber R, et al. Reduction of maternal-infant transmission of human immunodeficiency virus type 1 with zidovudine treatment. Pediatric AIDS Clinical Trials Group Protocol 076 Study Group. N Engl J Med. 1994 Nov 3;331(18):1173-80.

- Standard journal article: Corporate Author International Committee of Medical Journal Editors. Uniform requirements for manuscripts submitted to biomedical journals. N Engl J Med.1997 Jan 23; 336(4):309-16.

- Books and other monographs: Personal Author(s) Stewart M, Brown JB, Weston WW, et al. Patient-Centered Medicine: Transforming the Clinical Method. Thousand Oaks, California: Sage Publications; 1995.

- Books and other monographs: Corporate Author Wonca International Classification Committee. International Classification of Primary Care, ICPC-2. 2nd ed. Oxford: Oxford University Press; 1998.

\section{ENSURING A BLIND REVIEW}

To ensure the integrity of the blind peer-review for submission to this press, every effort should be made to prevent the identities of the authors and reviewers from being known to each other. This involves the authors, editors, and reviewers (who upload documents as part of their review) checking to see if the following steps have been taken with regard to the text and the file properties:

- The authors of the document have deleted their names from the text, with "Author" and year used in the references and footnotes, instead of the authors' name, article title, etc.

- With Microsoft Office documents, author identification should also be removed from the properties for the file (see under File in Word), by clicking on the following, beginning with File on the main menu of the Microsoft application: File > Save As > Tools (or Options with a Mac) $>$ Security $>$ Remove personal information from file properties on save $>$ Save.

- With PDFs, the authors' names should also be removed from Document Properties found under File on Adobe Acrobat's main menu.

\section{POLICY FOR USING ANY PUBLISHED MATERIALS}

Authors must seek approval from and acknowledge the MFP if they wish to use any published materials from this journal. Write to the Editorial Manager at email: editor.mfp@afpm.org.my

\section{ARTICLE PROCESSING CHARGES}

The Malaysian Family Physician does not charge submission, article processing, page or colour charges presently. The costs for services provided are funded by the Academy of Family Physicians of Malaysia and the Family Medicine Specialist Association. We retain the right to change this policy in the future, but this will only be done with due notice to all stakeholders.

\section{COPYRIGHT}

If the article is accepted for publication, the author agrees to transfer the copyright to AFPM.

Disclaimer: Malaysian Family Physician takes no responsibility for any of the content stated in the abstracts. The abstract book contains abstracts as provided by the authors. 
Plenary

Abstracts

Symposium Abstracts
1 Transforming Primary Care Service Delivery: Upholding Family Medicine Core Values Rozita Zakaria

2 Uberization of Primary Care: The defining healthcare trends of the 21st century Mohd Safiee Bin Ismail

3 Artificial Intelligence in Primary Care: Is It a Threat to Family Medicine Core Values? Nazrila Hairizan Nasir

4 Virtual Clinic in Primary Care: Embracing Innovation in Family Medicine Practices Associate Professor Dr. Tan Ngiap Chuan

5 Shaping Innovations in Long-Term Care for Stroke Survivors in the Community Professor Dr. Noorazah Abd Aziz

6 Evidence Based Medicine in the Digital Era: Challenges and Opportunities Professor Dr. Lai Nai Ming

1 Primary Care Governance \& Service Delivery Reform: Why, How and When? Norsiah Ali

2 Peka B40: A Public-Private Integration Model for NCD Screening \& Management Mohammad Husni Haji Ahmad Jamal

3 Health Financing Reform to Achieve Universal Health Coverage in Primary Care Muhammad Anis Abd. Wahab

4 SARSCoV-2 infection: what have we learn Suresh Kumar A/L Chidambaram

5 Mental Health Issues among COVID-19 survivors Amer Siddiq bin Amer Nordin

6 Primary health care and COVID-19: A journey beyond expectations Azah Abdul Samad

$7 \quad$ Eliminating Cervical Cancer in Malaysia: What does it take? Professor Dr. Woo Yin Ling

8 Hospice and Palliative Care in Malaysia Dr. Sylvia McCarthy

9 Hospice and Palliative Care Integration within community setting Dr Norhana Yazid

10 STEMI Network in Pahang Dr. Saniah Senik

11 Wound Care Management in Mengkibol Health Clinic: A Holistic Approach Dr. Hanihaselah Mohd Salleh

12 The Challenge in Leprosy Management in Rural Sarawak Dr. Maurice Steve Utap

13 Social media, pornography and sexual activities among Malaysian adolescents: the links Associate Professor Dr. Noor Azimah Muhammad

14 Doc, Is My Child Growing Well? Associate Professor Dr. Azriyanti Anuar Zaini

15 Stunted growth: nutritionist perspective Professor Dr Norhasmah Sulaiman,

16 The ScreenMen Mobile App: Empowering Hard-to-Reach Men to Screen Professor Dr. Ng Chirk Jenn

17 The EMPOWER-SUSTAIN Mobile App: Transforming Cardiovascular eHealth Intervention in Primary Care

Professor Dr. Anis Safura Ramli 
18 Three decades of Primary Care Evolution: Where are we heading?

Dr. Sri Wahyu Taher

19 Uncovering the Hidden Agenda in Mental Health Consultation

Dr. Navin Kumar Devaraj

20 Managing Clients Hot on ICE

Dr Fadzli Mohamad Isa

21 Perinatal Mental Health: Identifying and Managing Problems in Primary Care Dr. Umi Adzlin Silim

22 Sarcopenia: Symptoms, Causes and Dietary Management in Primary Care Professor Dr. Chan Yoke Mun

23 Exercise for Older Persons Professor Dr. Sazlina Shariff Ghazali

24 Role of Primary Care Physicians in Dementia Management Dr. Ho Bee Kiau

25 Home Birth: The Myth and The Reality Dr. Sakinah Sulong

26 Addressing Domestic Violence against Women Professor Dr. Sajaratulnisah Othman

27 Ravishing Face, Heavenly Body: Introduction to Aesthetic Medicine Associate Professor Dr. Adibah Hanim Ismail

28 Men's Health: Issues \& Challenges in Malaysia Dr George G. Mathew

29 The Needs of Prostate Cancer Survivors in Malaysia Professor Dr. Lee Ping Yein

30 Providing Inclusive and Holistic Services for the LGBT Community Professor Dr. Samsul Draman

31 Empowering Patients through Social Media: Benefits and Challenges Dr. Suhazeli Abdullah

32 Social Media Addiction: Its Impact \& Intervention Associate Professor Dr. Yee Hway Ann@ Anne Yee

33 Becoming a Celebrity Doctor: Is It Worth the Glamour? Dr. Imelda Balchin

34 Preventing Diabetes in the Offspring: What Does it Take? Professor Dr. David Whitford

35 Traditional, Complementary and Alternative Medicine Use among Patients with NCD: Benefits \& Harm

Professor Dr Ching Siew Mooi

36 Engaging Individuals to Undergo CVD Screening: What Are the Strategies? Associate Professor Dr. Cheong Ai Theng

37 Hepatitis C Management: A Reality in Kedah KK Dr. Habshoh Hat

38 Influenza: Forget me not Associate Professor Dr. Petrick Periasamy

39 COVID-19 Vaccination: new updates Dr David Strain 
Oral

Abstracts

1 A feasibility study of a school-based asthma programme in Malaysia conducted during COVID-19 pandemic

Dr Siti Nurkamilla Ramdzan

Family Medicine Specialist and Senior Lecturer

Department of Primary Care Medicine, University Malaya Medical Centre (UMMC)

2 Factors associated with sexually transmitted infections (STI) and STI rates at Teluk Intan Health Clinic (KKTI) from 2014 to 2018

Dr Suriata binti Daud

Family Medicine Specialist

Klinik Kesihatan Teluk Intan

3 Factors Influencing the Practice of Smoking Cessation Assessment and Management among Primary Health Care Doctors in Selangor Primary Healthcare Clinics (SCAAMDOC)

Dr. Beatrice Jee Ngee Ling

Family Medicine Specialist

Faculty of Medicine and Health Sciences, Universiti Putra Malaysia (UPM)

4 Intention and Practice on Breastfeeding Among Pregnant Mothers in Kinta District and Factors Associated with Practice of Exclusive Breastfeeding: A Cohort Study

Dr Nurul Mursyidah Shohaimi

Family Medicine Specialist

Klinik Kesihatan Buntong

5 The accuracy of detecting diabetic retinopathy (DR) using panoptic ophthalmoscope (PO) by primary care doctors at Klinik Kesihatan (KK) Sendayan, Negeri Sembilan Dr Nur Aishah binti Abdul Latiff

Medical officer

Klinik Kesihatan Sendayan

6 Mass-media intervention to improve breast cancer awareness and screening among women in Malaysia

Dr Mila $\mathrm{Nu} \mathrm{Nu}$ Htay

Assistant Professor

Department of Social and Preventive Medicine, Faculty of Medicine, University of Malaya (UM)

7 A Cross-Sectional Study On The Level Of Mindful Eating Among Type 2 Diabetes Mellitus Patients And Its Associated Factor

Nurul Hayati Chamhuri

Medical officer

Department of Primary care

Universiti Sains Islam Malaysia (USIM)

8 Baby Walker Usage Among Toddlers In Tangkak Johor: Have We Done Enough Dr Mohd Hazizul Ab Samad

Medical officer

Klinik Kesihatan Sungai Mati

9 Knowledge and attitude on childhood vaccination among healthcare workers in Hospital Universiti Sains Malaysia (HUSM).

Dr Ahmad Faiq Mukhtar

Family Medicine Specialist trainee

Department of Family Medicine, Universiti Sains Malaysia (USM)

10 Internet addiction among early adolescents during COVID-19 pandemic in the east coast of Peninsular Malaysia

Dr Lena Nanditha Sangaran

Family Medicine Specialist trainee

Department of Family Medicine, Universiti Sains Malaysia (USM)

11 Prevalence of Falls and Their Associated Factors Among the Hypertensive Older Adults in East Coast Malaysia

Dr Atirah Az-Zahra Abu Bakar

Family Medicine Specialist trainee

Department of Family Medicine, Universiti Sains Malaysia (USM) 
12 The interest and willingness to pay for a suitable takaful products among diabetes patients in Malaysia. A nationwide descriptive analysis in Malaysia Prof Madya Dr. Mohd Radzniwan B. A.Rashid

Family Medicine Specialist and Lecturer

Faculty of Medicine and Health Sciences, Universiti Sains Islam Malaysia (USIM)

13 A focus on religious and spiritual coping among Malay Muslim MSMs with HIV in Malaysia: A Qualitative Study

Associate Professor Dr. Farnaza Dato' Ariffin

Consultant Family Medicine Specialist

Department of Primary Care Medicine, Universiti Teknologi MARA (UITM)

14 Home medication management and its associated factors among long term stroke patient in a community based primary care clinic

Dr Mohd Hafizul Akmal Nordin

Family Medicine Specialist trainee

Department of Family Medicine, Universiti Kebangsaan Malaysia (UKM)

15 The prevalence of gestational diabetes, associated risk factors and feto-maternal outcome among antenatal women attending health clinics in Terengganu

Dr Rozimah Abdul Latif

Family Medicine Specialist

Klinik Kesihatan Merchang, Terengganu

16 Experiences of Family Medicine Specialist (FMS) in Providing Healthcare for Lesbian, Gay, Bisexual and Transgender (LGBT) Patients: A Qualitative Study

Dr Siti Noorfadhilah Bt Mohd Amin

Family Medicine Specialist trainee

Department of Family Medicine, Universiti Sains Malaysia (USM)

17 Utility and Usability of the EMPOWER-SUSTAIN Desktop and Mobile Apps ( among Primary Care Physicians and Patients with Metabolic Syndrome in Primary Care

Dr. Maryam Hannah Binti Daud

Phd Student and Medical Doctor Department of Primary Care Medicine, Universiti Teknologi MARA (UiTM), Malaysia

18 Level of acceptance of executive diploma programme among Medical Officers: A pre-study survey

Dr. Anu Suria Ganason

Family Medicine Specialist and Lecturer

Faculty of Medicine and Health Sciences, Universiti Sains Islam Malaysia (USIM)

Poster

Abstracts
1 U.N.G.G.A.S: Knowledge on HIVIAIDS among Adolescents in Kuala Lumpur \&

Putrajaya

Nazrie Sain

Health Education Officer

Health Department of Kuala Lumpur And Putrajaya

2 Long Term Benefit Of A Targeted Diabetes Education Program: The KK Chini Experience Dr. Nor Azam Kamaruzaman

Family Medicine Specialist and Lecturer

Department Of Family Medicine, International Islamic University Malaysia ( IIUM)

3 Association between screen time and sleep among children between 3 to 6 years old Dr Marina Antony Nicholson

Medical Officer

Klinik Kesihatan Kuala Lumpur

4 Prevalence of depression among type 2 Diabetes Mellitus patients in selected primary care clinics in Perak and it's associated factors.

Dr Najmi binti Anuar

Medical Officer

Klinik Kesihatan Pasir Pinji, Perak

5 Danger signs in unwell children under five: can the caregivers identify them?

Dr. Nor Azam Kamaruzaman

Family Medicine Specialist and Lecturer

Department of Family Medicine, International Islamic University Malaysia (IIUM) 
6 Effectiveness of Healthy Lifestyle Education in Diabetic Control in a Primary Care Clinic Dr Tay Chai Li

Family Medicine Specialist

Klinik Kesihatan Simpang, Perak

7 Foot care knowledge and self-care practices among diabetics in Penang: a primary care study

Dr Ong Jue Jing

Medical Officer

Klinik Kesihatan Batu Muda, Kuala Lumpur

8 Measuring Implementation Fidelity of The Family Doctor Concept (FDC) Program in Public Primary Care Clinics in Selangor, Malaysia

Dr Muhammad Alimin Mat Reffien

Doctor of Public Health (DrPH) trainee

Department of Community Health, University Kebangsaan Malaysia

9 Non-compliance and its associated factors to iron chelation therapy among transfusion dependent thalassaemia patients in Kelantan and Terengganu

Dr Allen Shiun Chat Chai

Family Medicine Specialist trainee

Department of Family Medicine, Universiti Sains Malaysia (USM)

10 Audit on Dyslipidemia management of Type2 Diabetes Mellitus patients in Changkat Jering Heatlh clinic, Perak

Dr Lai Mei Kuen

Medical Officer

Klinik Kesihatan Changkat Jering, Perak

11 Factors associated with high obstructive sleep apnea risk mong adult patients attending uitm primary care clinic

Dr Sharifah Azimah Bt Wan AI

Family Medicine Specialist

Klinik Kesihatan Kapit, Sarawak

12 Stress, anxiety and depression among antenatal women in Terengganu: The prevalence and associated factors

Dr Nurul Azreen Yusof

Family Medicine Specialist and Lecturer

Universiti Sultan Zainal Abidin, Terengganu

13 Epidemiological trends of NCD in Beladin, Pusa, Sarawak: A cross sectional study Dr Stephen Jeshua Manuel

Primer Officer

Pejabat Kesihatan Bahagian Betong, Sarawak .

14 The prevalence of depression and its associated factors among married female diabetic patients in Kuantan, Pahang

Dr Tan Kui Foung

Medical Officer Department of Family Medicine

International Islamic University Malaysia (IIUM)

15 Fetal Heart Coin: Cardiac Rhabdomyoma not to be missed

Dr Tan Kui Foung

Medical Officer. Department of Family Medicine

International Islamic University (IIUM)

16 Patient Profile and Drug Use in A Special Upper Respiratory Tract Infection Clinic Based in A Primary Healthcare Setting During Covid-19 Pandemic in Malaysia: A Cross Sectional Study

Dr Ooi Zhi Yin

Medical officer

Klinik Kesihatan Batu Pahat, Johor

17 Functional Disabilities and Its Associated Factors among Elderly Residents in Private Nursing Homes in Kuantan

Dr Mazilah Mazlan

Medical officer Department of Family Medicine

International Islamic University Malaysia (IIUM) 
18 Exploring how patient engagement is initiated in diabetes care

Dr Syahnaz Mohd Hashim

Family Medicine Specialist and Lecturer

Department of Family Medicine, Universiti Kebangsaan Malaysia (UKM)

19 A Retrospective Audit On Cloxacillin Prescription In Primary Health Care Facilities In Kuala Selangor

Dr Nurul Nadia Baharum

Family Medicine Specialist

Klinik Kesihatan Tanjong Karang, Selangor

20 Prevalence of severely elevated low-density lipoprotein cholesterol (LDL-c) in public primary care clinics in Klang Valley, Malaysia

Profesor Dr Anis Safura Ramli

Consultant Family Physician

Department of Primary Care Medicine, Universiti Teknologi MARA (UiTM)

21 Clinical detection of Familial Hypercholesterolaemia (FH) using Familial Hypercholesterolaemia Case Ascertainment Tool (FAMCAT), Simon Broome (SB) Criteria and Dutch Lipid Clinic Criteria (DLCC) in public primary care clinics in Klang Valley, Malaysia

Profesor Dr Anis Safura Ramli

Consultant Family Physician

Department of Primary Care Medicine, Universiti Teknologi MARA (UiTM)

22 Prenatal diagnosis and management of fetal hydronephrosis in primary care.

Dr Nadia Hamimah Binti Kamaludin

Family Medicine Specialist

Klinik Kesihatan Putatan, Sabah

23 Effective contraceptive practise and its associated factors among women with uncontrolled diabetes in Terengganu

Dr Hassan Basri Bin Mukhali

Family Medicine Specialist and Lecturer

University Sultan Zainal Abidin, Terengganu

24 Does system-based intervention reduce incidence of Type 2 diabetes and improve the metabolic profiles postnatally in women with history of gestational diabetes?

A prospective, randomized controlled study (SBI-GDM)

Dr Tan Siow Foon

Family Medicine Specialist

Klinik Kesihatan Pelabuhan Klang, Selangor.

25 Validity and Reliability of the Malay IOWA Infant Feeding Attitude Scale (IIFAS-M) among mothers with infants in Malaysia

Dr Hazwani Hanum Binti Hashim

Family Medicine Specialist Trainee

Department of Family Medicine, International Islamic University Malaysia (IIUM)

262 in 1: COVID-19 infection reveals tuberculosis

Dr Nur Izdiani

Medical officer

Klinik Kesihatan Taman Ehsan, Selangor

27 Internal Jugular Phlebectasia in Child; Now You See It Now You Don't

Dr Muhammad Zul Hazmi Ahmad Zawawi

Family Medicine Specialist trainee, Department of Family Medicine

International Islamic University Malaysia (IIUM)

28 Prevalence And Factors Associated With Psychological Distress Among Patients With Hypertension In A Primary Care Clinic

Dr Wen How Loke

Family Medicine Specialist trainee

Department of Family Medicine, Universiti Putra Malaysia (UPM) 
28 Prevalence And Factors Associated With Psychological Distress Among Patients With Hypertension In A Primary Care Clinic

Dr Wen How Loke

Family Medicine Specialist trainee

Department of Family Medicine, Universiti Putra Malaysia (UPM)

29 The effect of structured physical activity during pregnancy on maternal health and fetal outcomes: A systematic review .

Dr Farah Hanani Mohd Nor

Family Medicine Specialist trainee

Department of Family Medicine, International Islamic University Malaysia (IIUM)

30 The effectiveness of UNICEF/WHO 20-hour breastfeeding Course in Improving Health Professionals' Knowledge, Attitudes and Practice towards Breastfeeding in Malaysia: A quasi experimental study

Dr Nurjasmine Aida Jamani

Family Medicine Specialist and Lecturer

Department of Family Medicine, International Islamic University Malaysia (IIUM)

31 Incidence and factors associated with diabetic retinopathy among diabetic patients in Klinik Kesihatan Cheras

Dr Nadia Hamimah Binti Kamaludin

Family Medicine Specialist

Klinik Kesihatan Putatan, Sabah

32 Prevalence of Depression, Anxiety, Stress Symptoms and the Related Factors among Caregivers of Children with Autism Spectrum Disorder in Kuantan, Pahang Dr Puteri Fatin Nabilah Binti Megat Ahmed Tahwil

Family Medicine Specialist trainee

Department of Family Medicine. International Islamic University Malaysia (IIUM)

33 Prevalence of depression, anxiety and stress and its association with nicotine dependency among adult smokers attending primary care clinics

Prof. Madya Dr. Ambigga Devi S. Krishnapillai

Family Medicine Specialist and Lecturer

Universiti Pertahanan Nasional Malaysia (UPNM)

34 The Prevalence and Factors Associated with Mental Health Status among Youths in Malaysia

Dr. Fadzilah Binti Mohamad

Family Medicine Specialist and Lecturer

Department of Family Medicine,. Universiti Putra Malaysia (UPM)

35 Fracture risk (FRAX) and fall risk (FRAT) assessment in the elderly. Should we assess both to determine fracture risk?

Associate Professor Dr. Farnaza Dato' Ariffin

Consultant Family Medicine Specialist

Department of Primary Care Medicine, Universiti Teknologi MARA (UITM)

36 Development and validation of the Malay Self-efficacy, Knowledge, Attitude and Avoidance Practice towards Environmental Tobacco Smoke Questionnaire (SE-KAPETSQ) for mothers with children below 6 years old Associate Professor Dr. Farnaza Dato' Ariffin

Consultant Family Medicine Specialist

Department of Primary Care Medicine, Universiti Teknologi MARA (UITM)

37 Perceived barrier towards Hepatitis C management among Primary Care Doctors in Sabah

Dr Teh Rohaila Jamil

Family Medicine Specialist and Lecturer

Department of Family Medicine, Universiti Kebangsaan Malaysia (UKM)

38 Audit on Optimisation of Diabetic Control Over 5 Year Period (2016-2020) in Menglembu Health Clinic in Kinta District

Dr Marian Norlee

Medical Officer

Klinik Kesihatan Menglembu, Perak 
39 Audit of Insulin Injection Knowledge and Practices Among Diabetic Patients in UiTM Primary Care specialist Clinic, Selayang Campus

Dr Siti Fatimah Badlishah Sham

Family Medicine Specialist and Lecturer

Department of Primary Care Medicine, Universiti Teknologi MARA (UITM)

40 Clinical Audit of Management for Type 2 Diabetes Mellitus Patients in UiTM Primary Care Specialist Clinic, Selayang Campus

Dr Nik Aminah Nik Abdul Kadir

Family Medicine Specialist Trainee

Department of Primary Care Medicine, Faculty of Medicine, Universiti Teknologi MARA (UITM)

41 Hajj health examination for pilgrims with asthma in Malaysia: An ethnographic study Dr Rizawati Ramli

Family Medicine Specialist and Lecturer

Department of Primary Care Medicine, University Malaya Medical Centre (UMMC)

42 Case report: Trigeminal Herpes Zoster post Covid-19 vaccination. Is there an association? Dr. Anuar Sani

Senior Medical Lecturer

Faculty of Medicine \& Health Sciences, Universiti Sains Islam Malaysia (USIM)

43 Adolescents' Diet Quality at Home Food Environment: A Qualitative from mothers' perspective

Miss Suhaila Abdul Ghaffar

Nutritionist, Nutritional Science Programme

Faculty of Health Science, Universiti Kebangsaan Malaysia (UKM).

44 Vesicular Rash Post COVID-19 Vaccination . Is It Varicella Infection Related Or Side Effect?

Prof Madya Dr. Mohd Radzniwan B. A.Rashid

Family Medicine Specialist and Lecturer

Department of Primary Healthcare, Universiti Sains Islam Malaysia (USIM), Negeri Sembilan

45 Don't Underestimate Exudates: Management of a Traumatic Wound with Heavy Exudates Dr. Rachael Shamini Danasamy

Family Medicine Specialist

Klinik Kesihatan Bandar Tasek Mutiara, Pulau Pinang

46 Enhancing Healthcare Utilisation for the Asnaf During COVID-19 Pandemic: USIM Specialist Clinics Initiatives

Dr. Sharifah Najwa bt Syed Mohamad

Family Medicine Specialist and Lecturer

Department of Primary Healthcare, Universiti Sains Islam Malaysia (USIM), Negeri Sembilan

47 A Prompt Treatment of Renal Cell Carcinoma : The Coalition of The Masseuse, Primary Care Physician, Radiologist and Surgeon

Dr. Sharifah Najwa bt Syed Mohamad

Family Medicine Specialist and Lecturer

Department of Primary Healthcare, Universiti Sains Islam Malaysia (USIM), Negeri Sembilan

48 Prevalence of Primary Dysmenorrhea and Factors Associated Among Women at Kuala Selangor - A Cross-Sectional Study

Dr. Yafizah binti Yahaya

Family Medicine Specialist and Lecturer

Department of Primary Healthcare, Universiti Sains Islam Malaysia (USIM), Negeri Sembilan

49 Neglecting child neglect: A forgotten form of child maltreatment

Dr Chow Suet Yin

Family Medicine Specialist

Klinik Kesihatan Taman Medan, Selangor.

50 Modern contraceptive usage and its associated factors among Orang Asli women attending community clinics in east Pahang

Dr Muhammad Zul Hazmi Ahmad Zawawi

Family Medicine Specialist trainee

Department of Family Medicine, International Islamic University Malaysia (IIUM) 
51 Knowledge, attitude, and confidence in dementia management among primary care doctors in Malaysia - the importance of postgraduate training

Dr Mohamed Syarif Mohamed Yassin

Senior Lecturer and Family Medicine Specialist

Department of Primary Care Medicine, Universiti Teknologi MARA (UITM)

52 Attitudes of primary care doctors on depression: A cross sectional study in Terengganu Dr Nurul Izza Yunus

Family Medicine Specialist and Lecturer

Family Medicine Unit, Universiti Sultan Zainal Abidin

53 Development and validation of the Screen Dependency Scale (SDS) among pre-school children

Asst. Prof Dr. Azwanis Abdul Hadi

Family Medicine Specialist and Lecturer

Department of Family Medicine, International Islamic University Malaysia (IIUM)

54 Malay Mother's Views on Sexuality and Sexual Education to their Intellectual Disabilities Children (A Qualitative Study)

Dr Nawal Nabilah Kamaludin

Family Medicine Specialist trainee

Department of Family Medicine, Universiti Sains Malaysia (USM).

55 Knowledge, Attitude, Intention To Screen For Thalassaemia And Its Associated Factors Among Unmarried Individual Attending Premarital Course In Kota Bharu

Dr Muhammad Akmal Mohd Nor

Family Medicine Specialist

Klinik Kesihatan Sindumin, Sabah

56 Factors Associated with High-Sensitivity C-Reactive Protein and Interleukin-6 among Adults with Metabolic Syndrome in a Primary Care Clinic

Dr Jazlan Jamaluddin

Family Medicine Specialist trainee

Department of Primary Care Medicine, Universiti Teknologi MARA (UITM) 


\section{PLENARY ABSTRACTS}

\section{Plenary Abstract 1}

Transforming Primary Care Service Delivery: Upholding Family Medicine Core Values

\section{Rozita Zakaria ${ }^{1}$ \\ ${ }^{1}$ Precinct 18 Putrajaya Health Clinic, Wilayah Persekutuan Putrajaya}

The history of Primary care service in Malaysia has come a long way. Upon independence in 1957, Maternal and Child Health services came to fore as an essential component of the National Rural Health Development Programme. It has now transformed in phases from a vertical programme delivered through a 3-Tier System with only 1 medical officer in the Main Heath centre, to Health Clinics with a wide range of primary care services provided by medical officers and Family Medicjine Specialists. The year 1997 started off as the early years of Family Medicine where there is a metamorphosis of general practice at outpatient department into the specialty of family medicine.

Primary care which is the backbone of our health system is under immense strain. To meet the health challenges of the 21st Century and secure the future of family medicine, transforming Primary Care Service Delivery is inevitable. Three important strategies will be deliberated mainly Enhanced Primary Health Care, Virtual Clinic and Uberisation of Health Care services.

Virtual Directly Observed Treatment (V DOT) which is a personal initiative introduced to Klinik Kesihatan Putrajaya Presint 18 will also be discussed. V DOT is a modified treatment strategy from the World Health Organisation's Directly Observed Treatment whereby observing patients taking their anti TB treatment is done virtually using Virtual Clinic platform.

With transformation, the ways in which care is delivered will definitely change. Despite the changes, family medicine in the future will need to remain true to its core values. There is a need to define its own core values according to the local situation. Once the core values have been defined, it must be shared with the whole team so that the delivery of services will be congruent.

With the right tools, skills and investment, on top of upholding family medicine core values, family medicine specialists can continue to deliver world class patient care and being a Family Medicine Specialist can be the best job one can ever have.

\section{Plenary Abstract 2}

Urbanization of Primary Care: The Defining Healthcare Trends of the 21st Century

\section{Mohd Safiee Bin Ismail ${ }^{1}$ \\ ${ }^{1}$ Hospital Tuanku Fauziah, Perlis.}

Undoubtedly, future technology disruption will change PHC service delivery landscape to make it more efficient, accessible and support high quality primary care. The traditional facility based PHC services where face to face interaction occurred shall no longer dominate the ways health care are delivered. Nowadays, patients demand more convenient care within friendly environment in home and community setting. Uberisation of primary care service through usage of digital platform and mobile applications offers choice and save a lots of burdensome travel time in seeking care for those who can pay out of pocket. To gain the full benefit of this technology, issues relate to quality care, equal access to ICT (digital equity), digital literacy and regulation pose great challenges to country health system worldwide.

\section{Plenary Abstract 3}

\section{Artificial Intelligence in Primary care : Is it a Threat to family medicine Core Values}

\section{Nazrila Hairizan Nasir ${ }^{1}$ \\ ${ }^{1}$ Primary Care Section, Ministry of health, Malaysia}

Artificial intelligence is a term used commonly now in medicine. To put it simply, artificial intelligence is a field that enables problem solving by using computer science and robust data sets. However this concept is not new as it was introduced more than 70 years ago where Alan Turing described it as "thinking machines". With AI now, machines can perform a multitude of tasks like detecting retinopathy, skin cancer or lung nodules and predict outcomes like emergency department visits, opioid misuse and even deaths. The presentation would explore and discuss regarding the use of AI in the field of family medicine and whether AI would be an asset or a threat to the core values of family medicine mainly patient-doctor relationship/patient centred-care , comprehensiveness, holistic care and professionalism

\section{Plenary Abstract 4 \\ Virtual Clinic in Primary Care}

\section{Tan Ngiap Chuan ${ }^{1}$}

${ }^{1}$ Malaysia SingHealth Polyclinics, Singapore

The Covid-19 pandemic has provided unprecedented impetus in transforming traditional healthcare system to a tech-enabled virtual care model, including those in primary care.

SingHealth Polyclinics (SHP) comprise a network of public primary care clinics in eastern Singapore. It is an accredited institution for Academic Family Medicine The pandemic has escalated the pace of SHP care transformation. Telehealth has shifted care provision partially to a virtual platform. Video-based tele-consultation between the polyclinic physician and patients enables the continuity of chronic disease management. Video consultation is now extended to wound care and Direct Observed Therapy for patients with tuberculosis.

Patients with hypertension are supported by telemonitoring, telesupport and tele-treatment when they enroll into the PTEC Hypertension program. The on-going Optimum 
randomized controlled trial evaluates the health outcomes and cost-effectiveness of patients with diabetes mellitus over two years using an app operated on a private telehealth platform.

New tools are being evaluated for its deployment in the virtual space. A novel system integrating smart sensor, gamification and virtual coaching (STEADES) is being tested for its effectiveness in increasing smoking quit rate. Virtual reality (VR) technology has reduced anxiety in older children and their parents during immunization in a pilot trial. Separately, VR is being tested in an innovative assessment tool to evaluate the six domains of cognition in older adults.

The next step is to blend the virtual clinic initiatives with current clinical services to allow flexible, convenient and person-centered care to suitable patients without compromising on their safety and trust in SHP..

\section{Plenary Abstract 5}

Shaping innovation in long-term stroke care for stroke survivors in the community: A Multi-Disciplinary Approach

\section{Noor Azah Abd Aziz ${ }^{1}$ \\ ${ }^{1}$ Family Department of Family Medicine, Faculty of Medicine, UKM Medical Centre, Kuala Lumpur}

World Health Organization or WHO has defined stroke as 'rapidly developing clinical signs of focal loss of cerebral function, with symptoms lasting more than 24 hours or leading to death, with no apparent cause other than that of vascular origin. Stroke is a unique entity due to its evolution of its complications. These complications are complex, mostly influenced by various factors; either the patients' co-morbid conditions, current COVID-19 situation, or even external factors. The 2005 WHO Report identified stroke and coronary heart disease as major contributors for adult mortality, causing up to $20 \%$ of deaths worldwide. Stroke was the third highest cause national deaths in 2000, ranked after coronary deaths and pneumoniae. The further (or longer stroke care) which is one of the components in comprehensive stroke care was introduced early 2000, involving components of transfer of care, follow-up care, leisure intervention and community re-integration. The stroke providers now in consensus that this phase of longerterm stroke care should begin as soon as the patient is being discharged from the ward to the community and should involve a multi-disciplinary approach in managing the various needs of the patient and the family. The concept of longer-term care in primary care is new in Malaysia compared to the established care in hospitals. Nonetheless, stroke survivors in the community are currently facing various issues, most pressing is the issue with independence. Other issues include difficulty in accessing information, difficulty, and confusion regarding transfer of care process and lastly support in managing psychological changes after stroke. The shared care approach involving multi-disciplinary team is viewed as the most suitable approach for longer-term stroke care, as survivors at this stage require various care approaches to fulfill the various needs and issues of patient and the family. The Longer-Term Stroke Clinic (Klinik Lanjutan Strok) UKM Medical Centre was introduced in September 2008, as to fulfill the needs for longer-term stroke care in primary care setting. It is the first of its kind in Malaysia that provides comprehensive stroke care for the community, focusing on those who have been discharged from the hospital back to their homes.

\section{Plenary Abstract 6 \\ Evidence Based Medicine in the Digital Era: Challenges and Opportunities}

\section{Lai Nai Ming ${ }^{1}$ \\ ${ }^{1}$ Taylor's University School of Medicine}

Into its fourth decade, the understanding, practice and teaching of evidence-based medicine are still evolving. The digital era brings forth unprecedented ease in accessing information, but also accompanying challenges in recognising information that is useful and is closer to the truth as well as issues in application. Each step in the tenet of evidence-based medicine, namely, asking question, accessing, appraising and applying information has become laden with challenges as the generation, presentation and interpretation of evidence become more complicated, while the pressing need to make evidence plain and understandable to consumers remains.

In this talk, the speaker argues that part of the challenges in practicing evidence-based medicine is related to human attributes, be it of the individual, the team and the resultant environment at work. The discipline of focus, the trust and reliance on the team to enable efficient and effective retrieval, appraisal and incorporation of evidence, as well as the modification of work environment may provide a robust framework that can stand the continuing evolution of evidence-based medicine and medical practice in general.

\section{SYMPOSIUM ABSTRACTS}

Symposium Abstract 1

Primary Care Governance \& Service Delivery Reform: Why, How and When?

\section{Norsiah $\mathbf{A}^{1}$}

${ }^{1}$ Masjid Tanah Health Clinic Melaka1

Primary care is the provision of integrated, accessible health care services by physicians and their health care teams who are accountable for addressing a large majority of personal health care needs, developing a sustained partnership with patients, and practicing in the context of family and community.

Primary care has been regarded as the thrust of health care services worldwide. Primary care providers sit at the point of first contact. It is very crucial to make correct decisions at this level of care in order to ensure patient safety and good quality service delivery. Moreover, patients come in undifferentiated 
manner as certain symptoms could possibly reflect a few systems' clinical presentation. Hence working at this point of care can be very challenging. Therefore, a good primary care governance is deemed needed.

Primary care governance comprised of five main pillars: risk management \& clinical audit, continuous training \& education, evidence based, staffing \& staff management and patient \& carer centred. It offers the opportunity to all healthcare workers in primary care who actually directly involve in providing the services to take charge of the agenda to improve the health and health care of their local population while at the same time providing the accountability that is now expected. Primary care system varies as some countries are fully funded by government, some based on health care financing and some comprised of mixtures between government funded and private practice with out-of-pocket burden to patient. Malaysia belongs to the third category with very challenging issues especially in ensuring the five pillars of primary care governance are being followed fully. As a result, quality of care may jeopardize due to imbalance patient workload. When will the two wings of primary care providers merge and taken charge by primary care providers itself is still a big question mark as there are many factors and issues to be harmonized.

Over the years, there are improvement and promising future for implementation of primary care governance in this country. It will take strong clinical leadership to develop clinical governance to its full potential. Hopefully one day we will be there.

\section{Symposium Abstract 2}

PEKA B40: A Public-Private Integration Model for NCD Screening \& Management

\author{
Mohammad Husni J,2 \\ ${ }^{1}$ Department of Family Medicine, Faculty of Medicine, \\ University of Cyberjaya; ${ }^{2}$ Academy of Family Physicians of \\ Malaysia
}

Public-private partnerships are cooperative agreements between the public and private sectors working together by various mechanisms to achieve their common objectives. PEKA B40 is a health screening programme involving public health clinics and private Malaysian general practitioners (GPs) which was launched in 2019 by the Malaysian government. The purpose is for the early detection of noncommunicable diseases which are prevalent in the $40 \%$ lowpoverty sector of the population described as the B40 group. Although laudable, it has many shortcomings. Many GPs are still not receptive due to various reasons. A Public - Private Partnership committee was recently established between the Family Medicine Specialist Association of Malaysia and the Academy of Family Physicians of Malaysia to create a framework of bilateral cooperation. It is appropriate therefore for this committee to design a comprehensive program that is mutually beneficial for a better PEKA B40 platform. Two relevant studies are looked at in this context - the first is an expert panel recommendation for optimising non-communicable disease (NCDs) management in the ASEAN region whilst the other is an Indian study pertaining to designing a comprehensive NCD programme for hypertension and diabetes at primary health care level. By adopting the principles, outcomes and recommendations of such studies would be able to create a more effective PEKA B40 program in the Malaysian setting.

\section{Symposium Abstract 3}

\section{Health Financing Reform to Achieve UHC in Primary Care}

\author{
Muhammed Anis AW ${ }^{1}$ \\ ${ }^{1}$ National Health Financing Section (NHF) \\ Planning Division \\ Ministry of Health Malaysia
}

The current COVID-19 crisis has created an opportunity and provided momentum for reform in the health system. Governments should adopt a problem-based policy development approach in moving towards health financing reform. This begins with clarity on the objectives and goals to be achieved, which should be to improve population health, provide financial risk protection, and ensure citizen satisfaction, by achieving equity, efficiency, effectiveness, and responsiveness.

Regardless of the label of a country's health system, all systems perform similar functions in terms of health financing. This includes raising revenues for the health system, pooling these revenues on behalf of some or all of the population, and then transferring these resources to health care providers through strategic purchasing. Health systems then need to make policy choices, either explicitly or implicitly, regarding the benefit package or entitlements of the covered population.

A health financing reform focusing on primary care should be able to address Malaysia's epidemiological transition, as well as improve continuity, coordination, and comprehensiveness of health care, thereby improving health outcomes and overall patient experience.

\section{Symposium Abstract 4}

\section{SARS Cov 2 - What have we learnt so far}

\section{Suresh Kumar $\mathbf{C}^{\mathbf{1}}$ \\ ${ }^{1}$ Hospital Sungai Buloh}

Across the country the number of cases are coming down, however in the last few months the virus has taught us lots valuable lessons albeit not new ones. Old adages like "Prevention is better than cure" and 'a stich in time saves nine" are some that come to mind.

Moving forward, the post vaccination era is making us pivot once more. We need to deal with less sick patients but the numbers can still be overwhelming given that we can no longer keep non-covid services in the back burner.

We need to use the lessons we have learnt so far to chart a new course. Going back the pre-pandemic ways of doing things will be doing injustice the thousands who succumbed to this disease. 


\section{Symposium Abstract 5}

\section{Mental Health Issues Among COVID-19 Survivors}

\section{Amer Siddiq $\mathbf{A N}^{1}$ \\ ${ }^{1}$ Department of Psychological Medicine, Universiti Malaya}

Globally, the pandemic that is COVID-19 has resulted in massive loss of life and crippled economies across the globe. However, as we are all focused on tackling this pandemic, silently in the background, another equally devastating pandemic is growing insidiously - a mental health pandemic. This latter pandemic has not been highlighted, supported nor thought thoroughly as the former, and only recently noted on its negative effect. As we move towards the second year of the COVID-19 pandemic there is a growing number of reported mental health decline especially among the most vulnerable and, also increasing reports of suicide. One population that is often not mentioned are the survivors of COVID-19. A recent international study reported that nearly 1 in 3 COVID-19 survivors struggle with a neurological or mental health condition. Closer to home, it was estimated that amongst those who had COVID-19 in hospital, nearly $7-7.5 \%$ of them were found to have depression and anxiety. Moreover, for those who survived a difficult infection, survivors' guilt was reported. This presentation will explore further into this issue and attempts to highlight known risk factors on mental health risk amongst people with COVID-19 apart from possible solutions to assist those who have survived to lead better meaningful lives.

\section{Symposium Abstract 6 \\ Primary Health Care and COVID-19: A Journey beyond expectations}

\section{Azah AS \\ ${ }^{1}$ Seksyen 7 Health Clinic, Shah Alam, Malaysia}

The spread of COVID-19 is one of the biggest challenges the global community has faced. It has been almost 2 years since the Covid-19 pandemic hit Malaysia and has presented unprecedented challenges in primary health care as most of the health workforce is focused on the activities related to COVID-19. In primary care settings, physicians were encouraged to triage medical appointments, prioritize services, reschedule chronic disease appointments, and increase their use of virtual care. This has resulted in a reduction of visits to the outpatient clinics and delay doing routine preventive health care screening. Assessment should be made whether the changes in how family medicine is being practiced have impacts on the health outcomes of the patients.

With this COVID-19 pandemic, a visit to a family medicine specialist would be a one-stop centre as we screen, diagnose, monitor and see them post recovery. As the disease evolves, we encounter many post covid patients at the clinics. Long-term manifestations are increasingly recognized in COVID-19 patients, with systemic clinical presentations affecting a wide range of organs and systems. More training and support staff in rehabilitation will avoid fragmented care. Primary care entered the crisis facing greater demand with fewer resources than the tertiary centre. Playing its role as the first contact with the health system, it has successfully adapted and comply with the new norms while preserving and expanding care for the needs of patients and communities over the past year. The COVID-19 crisis demonstrates the importance of placing primary health care at the core of health systems, both to manage an unexpected surge of demand and to maintain continuity of care for all. An organized primary care system, well equipped with digital technologies and multidisciplinary teams helps deliver a successful health system response.

\section{Symposium Abstract 7}

Eliminating Cervical Cancer in Malaysia: What does it take

\section{Woo $\mathbf{Y L}^{1}$}

${ }^{1}$ Department of obstetric \& Gynaecology, University Malaya

In November 2020, the World Health Organization launched the strategy to accelerate the global effort to eliminate cervical cancer. The strategy involves vaccinating $90 \%$ of our adolescent girls with HPV vaccine by the age of 15 , screening $70 \%$ of our women at the ages of 35 and 45 with a HPV testing and ensuring that $90 \%$ of those with abnormal tests receive the appropriate treatment. Malaysia made a commitment to work towards those targets. This is achievable as we now have very effective tools to implement in a country such as Malaysia. Furthermore, Malaysia has had a very successful HPV vaccination program since 2010 .

Like many countries, the uptake of cervical screening has been low despite it being free in governmental clinics since 1969. And most women do not undergo regular screening. The advent of HPV testing via self-sampling is set to transform the landscape of cervical screening. Unlike the previous conventional pap smear which requires an uncomfortable speculum examination to obtain cervical cytology every 3 years, HPV testing from a low vaginal swab (Floqswab) offers much higher sensitivity in identifying cervical intraepithelial neoplasia.

Malaysia is well-placed to achieve the cervical cancer elimination targets if we start incorporating HPV testing among women who are eligible. The ability to perform selfsampling for HPV testing only as few as twice in a woman's lifetime is achievable. Ensuring proper communication between women and healthcare professionals will build trust and a workable system to ensure that everyone is aligned with this goal.

\section{Symposium Abstract 8}

\section{Hospice and Palliative Care}

\section{Dr Sylvia McCarthy ${ }^{1}$ \\ ${ }^{1}$ Hospis Malaysia}

This talk gives an overview of how palliative care has developed in Malaysia in the last 20-30 years alongside 
some of the developments in the understanding of palliative internationally. The evidence that early palliative care benefits patients with all life limiting illness will be discussed as well as the evidence around patients' palliative care needs. Most patients wish to spend as much of their time as possible in the community and their preferred place of death is usually home. Family Medicine and Palliative care have much in common and in many ways Family Medicine is ideally placed to respond to the need to provide palliative care in the community however there are challenges. Using cases, some of the issues facing patients with life limiting illness in the community will be highlighted. Finally there will be an overview of the work done by Hospis Malaysia and a discussion of some of the challenges that lie ahead if we are to develop truly patient centred care that enable patients to be cared for in their preferred place at the end of life.

\section{Symposium Abstract 9}

\section{Hospice and palliative care integration within a} community setting

\section{Dr Norhana $\mathbf{Y}^{1}$}

\section{${ }^{1}$ Klinik Kesihatan Chendering, Terengganu, Malaysia}

Palliative care is a specialised field for terminally ill patients to achieve the ultimate goal in providing holistic care and improving quality of life for them and their families. Collaboration between community palliative care and hospital-based palliative care is essential to maintain the continuity of care. This is accomplished by delivering consultation services in hospital or hospice organisation and providing home care team in the community. It varies in different state, depending on available resources they have but the goal remains the same. Challenges present in every flow of management but it can be ironed out with creativity and empathy. For certain issues highlighted in Terengganu in managing palliative patients, it shows that the integration of hospice organisation and family medicine specialists with modifications done to suit our current situation in COVID-19 pandemic are proved to be beneficial

\section{Symposium Abstract 10}

\section{STEMI Network in Pahang}

\section{Saniah $\mathbf{S}^{\mathbf{1}}$}

\section{${ }^{1}$ Klinik Kesihatan Maran, Pahang}

Pre-hospital thrombolysis (PHT) is an effective management strategy in patients with ST-segment elevation myocardial infarction (STEMI). PHT is safe and beneficial in remote area, distant from Cardiac Catheterisation Lab. The Malaysian CPG on STEMI 2019 recommended PHT when trained doctor/personnel are available and transport time to a PCI-capable centre is expected to be prolonged. Other option is nearest in-hospital thrombolysis.

The benefit of early treatment in STEMI is to reopen the blocked coronary artery as soon as possible to limit myocardial damage and preserve heart function. Primary PCI is superior to fibrinolytic therapy as a reperfusion strategy. However, in patient who present within 3 hours onset and at low risk, both treatment strategies appear to have similar benefits. Door to needle (DNT) time is shorter with prehospital thrombolysis.

PHT was started in Pahang since 2018. It was a collaboration between Departments of Emergency Medicine, Family Medicine, Cardiology/Medical and Pharmacy. It was supported by The State Health Director and Deputy Director as well as District Health Officer. The challenges in starting this programme were knowledge of the staff, limited ambulance with ECG, no thrombolytic agent in health clinics and distance to hospital. A few strategies to overcome the problems included I. Hands-on training (PHT and ALS workshop), II. Utilization of ALS ambulance, III. 'Thrombolytic pack' in health clinic, IV. Simplify referral system and collaboration between health clinic and hospital. Among positive outcomes of this STEMI Network programme were: I. Reperfusion @ success of thrombolysis achieved in more than $80 \%$ of cases without major complication. II. Mortality in 30 days was less than 5\%. III. The availability of Tenecteplase and Streptokinase in health clinic involved. IV. Medical equipment was upgraded such as Defibrillator Machine and Syringed Pump. V. Primary Health Care personnel being trained for Advanced Life Support.

In conclusion, DNT time was significantly reduced with PreHospital Thrombolysis. Successful reperfusion was achieved with neither increase in mortality rate nor complication of therapy. PHT was safe and feasible if given by well-trained doctor and dedicated mobile retrieval team. A good team work made the programme successful.

\section{Symposium Abstract 11}

\section{Wound Care Management In Mengkibol Health Clinic: A Holistic Approach}

\section{Hanihaselah MS ${ }^{\mathbf{1}}$ \\ ${ }^{1}$ Klinik Kesihatan Kulai, , Johor,}

The method of wound care has enhanced tremendously and evolved over the years. In Malaysia, for many years wound care is one of services that has been provided at primary health clinic but focusing only on wound cleansing and dressing. The number of patients came for wound dressing has increased overtime with the increased number of road-traffic accidents, increased prevalence of diabetes complications and other chronic diseases such as stroke and cancer. The development of new concepts into wound assessment and healing process, the availability wide variety of effective wound dressing materials triggered the interest of primary health care providers to this new method of wound care.

However, modern wound care cannot be implemented wisely due to many limitations - shortage of manpower, lacking of knowledge and skills on modern wound care dressing among health care providers, limited financial support, inadequate infrastructures and the availability of modern wound dressing products. To optimize the quality of wound care services with the limitations mentioned, few important and drastic 
interventions need to be plan and implemented with the full support from the top management in the Ministry of Health, at state and districts level. The Kluang District Wound Care Team is very proactive in implementing a well-structured, Holistic and Systematic Management of Wound Care in Primary Health Clinics in order to improve the quality of wound care services at the health clinic through efficient system and effective treatments. This holistic approach of wound care emphasizes on comprehensive patient care, established wound care team, organizing wound care trainings, establish wound care clinic, develop wound care registry, improve the defaulter tracing system, with multi-disciplinary integration and community empowerment.

Managing wounds with modern dressing techniques in primary health care are very challenging but shown to be effective to provide comprehensive care, proven shorten the time of wound healing, reduce the dressing load and improve the quality of life of the patients with wound and their caregivers.

\section{Symposium Abstract 12}

\section{The Challenges in Leprosy Management in Rural Sarawak}

\section{Maurice $\mathbf{S U}^{1}$ \\ ${ }^{1}$ Klinik Kesihatan Tudan, Miri, Sarawak}

Leprosy, or Hansen's disease, is a chronic infectious disease caused by acid-fast bacillus Mycobacterium leprae. It can be treated with multidrug therapy (MDT). Leprosy can cause permanent damage and disability to the skin, nerves, limbs and eyes if left untreated. Elimination status of leprosy is defined as prevalence of less than one case per 10,000 population. Elimination status has been achieved in Malaysia, Sarawak \& globally in the year 1994, 1996 and 2000 respectively. Despite achieving the elimination status, the annual prevalence rate of leprosy among the indigenous Penan in Sarawak is high at $5 \cdot 5$ case per 10,000 population. The Penan community is a part of the Dayak group in Sarawak with a population size of 16,281 in the year 2010 who inhabits the highlands of tropical forests which are less accessible and most of their dwellings are located between river basins. In this lecture, we will discuss the clinical diagnosis and management of leprosy, sharing experience and challenges from the active case detection of leprosy among the indigenous Penan in rural Sarawak and conceptual framework on the reason for the high prevalence of leprosy among the Penan. In conclusion, leprosy is a neglected tropical disease and is still endemic among the Indigenous Penan in rural Sarawak

\section{Symposium Abstract 13}

Social media, pornography and sexual activities among Malaysian adolescents: the links

\section{Noor Azimah Muhammad ${ }^{1}$ \\ ${ }^{1}$ Department of Family Medicine, Faculty of Medicine UKM}

Among the cases involving adolescents that are commonly seen in Primary Care Clinic include adolescent pregnancy as well as behavioural problems like bullying, school truancy and smoking related issues. Only a few of them present with social media addiction, cyberbullying or even sexually transmitted diseases. On the other hand, local data also has shown 5 in 6 of our young people below 20 years are active internet users and most of them own mobile devises like smartphone. Social media has turned to be an important platform to our adolescents to interact with one another. It is expected there will be higher usage during this current Covid-19 pandemic. This is a great concern for public health as anecdotal observation has shown that risky activities including sexual activity are partly influenced by their exposure from the internet. Thus, this presentation aims to review and provide local evidence on the relationship of the three main issues: social media use, pornography and sexual activities involving our adolescents. As for Family Physicians, it is prudent to have a clear understanding on the relationship in order to provide the best care to our adolescents of the current millennium.

\section{Symposium Abstract 14}

Doc! Is my child growing well?

\section{Azriyanti AZ}

${ }^{1}$ Department of Paediatrics, Faculty of Medicine, University of Malaya.

In 2017, UNICEF has reported $21 \%$ of Malaysian children below 5 are stunted. Stunting leads to risk of becoming short adults. In 2015, it is reported that the average height of Malaysian women is $155 \mathrm{~cm}$ and the average adult height for men is $167 \mathrm{~cm}$. Malaysian height ranked at 152 of 200 for women and at 137 of 200 for men comparing countries across the world. Being short has many disadvantages as adults and more so when they are younger.

There are many risks associated with poor growth in children. Born small for gestational age (SGA), chronic medical conditions, poor dietary habits and malnutrition, poor socioeconomic factors, environmental triggers and endocrine disorders are amongst many others. Parents and health care professionals (HCP) need to be vigilant and monitor children's growth regularly. Early recognition and diagnosis are essential for early intervention. Recognising children at risk of stunting is a responsibility of primary care physicians and general paediatricians. Appropriate early intervention e.g nutritional advice would be beneficial and referral to a subspecialty may be indicated if the child continues to have poor growth.

\section{Symposium Abstract 15}

\section{Stunted Growth: Nutritionist Perspective}

\section{Norhasmah $\mathbf{S}^{1}$ \\ ${ }^{1}$ Department of Nutrition, Faculty of Medicine and Health Sciences, Universiti Putra Malaysia}

Childhood stunting is recognized as one of the major global issues requiring urgent attention. Stunting is a sign of past 
or chronic undernutrition and cannot measure short-term changes in undernutrition. Nationally, there is an increased trend for stunting in young children. The prevalence of stunting increased in the previous decade (NHMS 2006 NHMS 2016) for infants below 6 months, children 12-23 months of age and children under 5 . The national prevalence of stunting significantly increased from $8.9 \%$ in 2006 to $14.0 \%$ in 2016 while in children $12-23$ months of age, the prevalence of child stunting significantly increased from $8.9 \%$ to $17.0 \%$. In all children under 5 , the prevalence of stunting increased from $17.5 \%$ to $20.5 \%$ in the period between 2006 and 2016 .

Stunted is a manifestation of maternal and child malnutrition which is the effect of complex interactions between households, environment, socioeconomics and culture. It involves three main factors comprising immediate causes (inadequate dietary intake, disease), underlying causes (household food insecurity, care and feeding practices, access to health services and healthy environment) and basic causes (sociodemographic, human capital and financial, culture). Stunted has long -term effects on the lives of individuals and society. Stunted children will experience impairment in physical growth and cognitive decline, while stunted children in adulthood will experience decreased productivity and increased risk of degenerative diseases/metabolic disorders such as type 2 diabetes, high blood pressure and cardiovascular disease.

The concept of the first 1,000 days was introduced and integrated into activities related to maternal nutrition to promote and improve nutrition for mothers and children. It is focusing on nutrition pre pregnancy, during pregnancy, after birth and complementary feeding. Thus, good nutrition in the first 1,000 days of life, which refers to the critical period between a woman's pregnancy and her child's second birthday is vital to prevent childhood stunting.

\section{Symposium Abstract 16}

\section{The ScreenMen mobile app: Empowering hard-to-reach} men to screen

\section{Ng Chirk Jenn ${ }^{1}$ \\ ${ }^{1}$ Department of Family Medicine, Faculty of Medicine, Universiti Malaya}

Evidence-based screening improves early detection of diseases, reduces disease burden, improves quality of life and lowers healthcare cost in the long run. However, screening uptake, especially in men, remains consistently low compared to women across the globe. Men would go for healthchecks when they fall ill, not when they are healthy and asymptomatic, partly due to the lack of awareness of the concept of screening.

In Malaysia, most screening happens opportunistically and might not be evidence-based. Therefore, a more tailored, evidence-based, accessible and inexpensive approach to screening is needed. The ScreenMen web app (http:// screenmen.org) is an open-access, personalised screening tool that aims to make screening accessible to working men; it was developed based on up-to-date clinical evidence, men's behavioural theories, and design thinking principles.

This paper is a synthesis of findings from several research studies and it aims to share the motivation and challenges in developing ScreenMen, how it could change men's screening intention and uptake, and the implementation outcomes of ScreenMen at a public health clinic.

\section{Symposium Abstract 17 \\ The EMPOWER-SUSTAIN Mobile App: Transforming Cardiovascular eHealth Intervention in Primary Care}

\section{Anis Safura $\mathbf{R}^{1}$ \\ ${ }^{1}$ Universiti Teknologi MARA, Malaysia}

Cardiovascular diseases (CVD), such as coronary artery disease $(\mathrm{CAD})$ and stroke, are the leading causes of death worldwide, contributing to 17.9 million deaths every year. In Malaysia, CVD is responsible for $35 \%$ of all deaths with CAD contributing $15 \%$ and stroke $8 \%$. Meanwhile, $18 \%$ of all deaths in 2020 were attributed to premature CVD deaths in the age group of 41 to 59 years old. Four major cardiovascular (CV) risk factors contributed towards CVD, namely hypertension, type 2 diabetes mellitus, dyslipidaemia and obesity. The rising prevalence of CV risk factors among Malaysians over the last 3 decades contributes towards this premature mortality. Exponential use of mobile phones in middle-income countries such as Malaysia unlocks the potential to transform $\mathrm{CV}$ risk factors management in primary care using digital health technology. In view of this, the EMPOWER-SUSTAIN Self-Management Booklet $@$ was converted into the EMPOWER-SUSTAIN Mobile App@ to suit the needs of the younger generations. Prototype development and refinement of the EMPOWERSUSTAIN Mobile App $@$ has recently been completed. The EMPOWER-SUSTAIN Mobile App ( is a hybrid app built using Ionic 4.0 with TypeScript as the coding language. The app runs in a low-level mobile browser shell e.g. iOS's UIWebView or Android's WebView. The EMPOWERSUSTAIN Mobile App $($ is a web page running in a native app shell. It is the first comprehensive app developed in Malaysia to be used by patients with multiple CV risk factors with the ultimate aim to prevent CVD. Our research team has won several Gold Medals at national innovation competitions for this novel invention.

\section{Symposium Abstract 18}

\section{Three decades of primary care evolution: where are we heading?}

\section{Sri Wahyu $\mathbf{T}^{\mathbf{1}}$}

${ }^{1}$ Klinik Kesihatan Simpang Kuala, Kedah

The family medicine fraternity was born officially in 1997. It was a revolutionary advancement in Ministry of Health Malaysia because that was the year for the first time ever, the population in the community will be cared by a clinical specialist in public health sector. Primary care is the thrust of 
the health system and literally the family medicine fraternity becomes the center to every aspect of disease management. Over the years, family medicine specialists (FMS) have developed primary care to an utmost level where people now say 'we cannot move forward without FMS'. Family medicine fraternity has gone beyond the clinic jurisdiction and now encroaching into specified care, spreading its wings into areas nobody has the gut to venture. FMS have been involved in numerous aspects of primary care variability and evolution. However, maintaining the core business of primary care. Research opportunity in primary care is tremendous. When COVID-19 savaged the country in 2020, FMS have no choice but to take up the responsibility and challenge of being involved in COVID-19 infection and prevention control at primary care. The resilience characteristics of FMS have held the fort firmly and superbly. The community's interest and future are in the hands of the FMS. Have we achieved enough? Are we satisfied with the current status? Do we thirst for more? Are we capable to face further challenges? Where do we place ourselves among other fraternities? Are we ready to leave our comfort zone? Will digitalization facilitate the future career pathway of FMS? Do we have a strategy?

\section{Symposium Abstract 19}

\section{Uncovering the Hidden Agenda in Mental Health Consultation}

\section{Navin Kumar Devaraj ${ }^{1}$ \\ ${ }^{1}$ Department of Family Medicine, Faculty of Medicine and Health Sciences, Universiti Putra Malaysia}

A 'Hidden Agenda' is an objective of an action or behaviour that isn't openly admitted or known, or visible in the consultation. It's a result you want to achieve, a goal of acting or behaving, but a result or goal that you are hiding from yourself and/or from others. A more complicated definition comes from the psychoanalytic theory, which defines hidden agenda as wishes, impulses, aims, and drives of which the self is not often aware. Examples of behaviour produced by unconscious motivation are purposive accidents, slips of the tongue, and dreams that express unfulfilled wishes.

It is not only psychiatric symptoms that patients may be reluctant to discuss due to fear and anxiety or even stigma. In primary care practices, we have been struck by how often patients may mention, almost in passing or leaving the consultation room, a lump in the breast or the chest pain they had climbing stairs. These comments are usually accompanied by the all too common phrase of "by the way". Physicians need not only to be attuned to the reality that patients may have a hidden agenda, but also need to arm themselves various strategies to try to unmask that agenda and the time to address issues raised. This can be especially difficult in a busy primary care practice. Despite this challenge, it is deeply gratifying to the clinician to uncover a patient's hidden agenda as then patient's health does often improve after this detective work is done.

This talk will delve into two real life cases that showcase the importance of unmasking the hidden agenda in delivering holistic care and helping our patients, primarily those with psychiatric disorders.

\section{Symposium Abstract 20}

\section{Managing Clients Hot on Ice}

\author{
Mohd. Fadzli MI ${ }^{1}$ \\ ${ }^{1}$ Psychiatry Addiction Unit (PAdU), Department of Psychiatry \\ \& Mental Health \\ Hospital Kuala Lumpur
}

Call it what you want- ice, syabu, batu, crystal or other related names, these are methamphetamines, and they are substances in the Amphetamine-type Stimulant (ATS) group. The number of methamphetamine use disorder cases presented to health and medical facilities have been increasing and this is also true for the cases of physical and psychological complications. Unfortunately, many medical and healthcare professionals are still struggling with the management of patients with methamphetamine use disorder whether they present in acute intoxication, withdrawal or when patients requested for their methamphetamine addiction to be medically managed.

Intoxication from methamphetamine will go off as it is a time-based phenomenon similar to other substance intoxication but acute behavioural disturbances following the intoxication may need symptomatic pharmacotherapy management. Similarly, symptomatic pharmacotherapy management and reassurance is the core of withdrawal management. Unfortunately, methamphetamine use disorder does not have any pharmacotherapy as a maintenance or relapse prevention agent.

Brief Intervention (BI) can be the first-line psychotherapeutic interventions followed by more complex and structured psychotherapy such as as Cognitive Behavioural Therapy (CBT) or Contingency Management (CM). The addiction clinic in Hospital Kuala Lumpur practices the Modified Brief Cognitive Behavioural Intervention (mBCBI) which is tailored to suit the local Malaysian clinical practice, while at the same time trying to be flexible to the patients so that patients can stay longer in the treatment system.

\section{Symposium Abstract 21 \\ Perinatal Mental Health: Identifying And Managing Problems in Primary Care}

\section{Dr Umi Adzlin Silim ${ }^{1}$ \\ ${ }^{1}$ Department of Psychiatry, Hospital Serdang}

Perinatal mental health problem is defined as any mental health problem that occurs during pregnancy and in the first 12 months following childbirth. Depression and anxiety disorders are the most frequent, but the less common postpartum psychosis is unique to postpartum. Complex and severe mental illnesses such as schizophrenia or bipolar disorders are low in prevalence but in need of intensive and collaborative care. Alongside mental disorders, there are also various mental health problems that can be experienced 
throughout the perinatal period, such as dealing with unintended pregnancies, coping with medical illnesses and obstetric difficulties, and grieving of perinatal losses.

Primary care services being the first point of contact for women when they are pregnant or planning to conceive, are playing crucial roles for women's overall mental health care in the perinatal period. The care includes 1) providing pre-pregnancy advice and care, which is particularly needed for women with a complex or severe current or past mental health problem, including liaising with psychiatry services, 2) identifying, assessing, and monitoring mental health issues 3) providing suitable mental health interventions 4) making referrals to psychiatry and mental health services.

There is a pressing need for effective care because of the impact they can have on both women and babies. The delivery of care should be collaborative, person-centred, compassionate, and supportive. Evidence-based interventions will provide a substantial long-term gain with meaningful and lasting effects for the wider family.

\section{Symposium Abstract 22}

\section{Dietary Management of Sarcopenia: a dietitian's perspective}

\section{Chan YM ${ }^{1}$ \\ ${ }^{1}$ Department of Dietetics, Faculty of Medicine and Health Sciences, Universiti Putra Malaysia}

Muscle health is important for the functionality and independence of older adults. Loss of muscle mass and strength, a condition known as sarcopenia, is gaining its popularity attributed to the increased older adult population in developed and developing countries including Malaysia. Several recent studies illustrate the magnitude of the above problem and reinforce the appropriate preventive measures at the primary care level. Etiology of sarcopenia is multifactorial, with low physical activity engagement and poor nutritional status are commonly known risk factors that are highly modifiable. Suboptimal diets and poor nutritional status are prevalent among the older adults, and therefore proper dietary management is important for both the prevention and treatment of sarcopenia. In this paper, the role of nutrition (single nutrients and dietary patterns) will be reviewed, along with their ability to preserve muscle mass and optimize physical performance. This presentation will also shed information on how research findings can be translated and incorporated into dietary management in optimising muscle health of older adults.

\section{Symposium Abstract 23}

\section{Exercise For Elderly}

\section{Sazlina SG1 \\ ${ }^{1}$ Faculty of Medicine and Health Sciences, Universiti Putra Malaysia}

Exercise for older persons has many health benefits to enable them to stay healthy and independent. This leads to longevity and improved quality of life, which allows older persons to remain productive even at later age in life. It is recommended that older persons engaged in at least 150 to 210 minutes of moderate intensity of physical activity in a week and to avoid sedentary behaviour. Healthcare providers plays an eminent role to facilitate older persons in achieving this goal by ensuring they are safe to exercise and they exercise safely. The initial steps in the assessment include assessing the physical activity level, determining the readiness to behaviour change and safety screening, which include preparticipation health screening and risk stratification. Most older persons are safe to start on low to moderate intensity exercise. The components of exercise prescription uses the acronym FITT-PRO, which stands for Frequency, Intensity, Time, Types and PROgression. Gradually increasing the frequency to almost everyday of moderate intensity physical activity leads to greater health benefits such as cardiovascular and fall risk preventions, improved disease control and enhanced psychosocial wellbeing. Doing bouts of at least 10 minutes of moderate intensity exercise intermittently over the day cumulatively to 30 minutes has similar health benefits to exercise performed continuously in one session. The best type of exercise should be the type of activity that fits the older persons, their motivations and their daily lives. It is imperative that older persons properly and safely progresses in an exercise training to minimize risk of injury and they should be regularly evaluated to adjust the prescription. Optimising behaviour change to adopt and adhere to exercise involves addressing barriers, identifying motivations and setting short- and long-term goals, which can be done using the 5As approach.

\section{Symposium Abstract 24 \\ Role of Primary Care Physicians in Dementia Management}

\section{Ho BK ${ }^{1}$ \\ ${ }^{1}$ Klinik Kesihatan Bandar Botanik, Klang, Selangor}

This symposium aims to define the role of primary care physicians in key aspects of dementia management in outpatient setting. Dementia management is often complex and associated with various medical, psychological \& social implications. Primary care physicians play important roles in dementia care, from prevention of dementia to overall management after diagnosis. They are usually the first point of patient's contact and provide care from early to late stages of dementia. They usually have a long-term understanding of the medical, psychological \& social situations of the patients and families. However, early dementia is often difficult to distinguish from normal ageing process in primary care. The role of primary care physicians for timely dementia detection and diagnosis is important to ensure early interventions, provide carers support and might reduce the rates of institutionalisation. Various challenges/ barriers faced by primary care physicians in providing dementia care in primary care which might lead to delays in diagnosis $\&$ active dementia management, for examples: insufficient time 
for comprehensive assessment, difficult to access secondary care, inadequate community resources, lack of knowledge or skill for appropriate dementia screening, diagnosis, and management. A variety of quality improvement strategies can be developed or adopted to address these issues and challenges.

\section{Symposium Abstract 25}

\section{Home Birth: The Myth and The Reality}

\section{Sakinah $\mathbf{S}^{\mathbf{1}}$}

${ }^{1}$ Klinik Kesihatan Masai, Johor

Safe delivery is a delivery process which is attended by a skilled and trained medical staffs. Though the majority of the deliveries occurred in healthcare facilities, there are certain numbers of women who choose to deliver at home for various reasons.

In several Western countries, home deliveries are planned and the process is well integrated into the health care system of that countries. This situation has been observed in Canada, Netherlands, New Zealand, Sweden, United Kingdom and Iceland. Netherlands has recorded as high as $20 \%$ of planned home deliveries in the year of 2013 and in New Zealand, the number was at 3.3\% in 2011. Malaysia has been seeing the trend emerging since 2012 and has been gaining popularity among mothers who want to have the same experience as their counterpart in the Western countries.

Therefore, there is a need to study the safety of this practice in Malaysia whether it is practical to carry out the same approach to meet the demand of our mothers here. We are going to see how it was done in the countries which was mentioned previously. We are also going to study what is the evidences on it's safety, should we plan to incorporate such practice in Malaysia.

Would it be a myth to expect a safe home delivery in Malaysia or whether it is achievable in our current setting?

\section{Symposium Abstract 26}

\section{Addressing Domestic Violence against Women}

\section{Sajaratulnisah $\mathbf{O}^{1}$ \\ ${ }^{1}$ Department of Primary Care Medicine, Faculty of Medicine, UM}

It is a syndemic for domestic violence! Since the declaration of the COVID-19 pandemic in March 2020, there have been reports of a surge in distress telephone calls to the helpline services worldwide. The measures to minimize the spread of COVID-19 has led to isolation which then increases the risks of violence within an intimate relationship. These include psychological distress, stress, loneliness, post-traumatic stress disorder, anger, sleep disorder and substance use. The victims' difficulty to access resources for help, fear of contracting the COVID-19, perception that minimize their issues and wanting to avoid burdening an already overwhelmed health system are some of the reasons to have led to reducing presentation of victims to health facilities. The reorientation from face-to-face service delivery to online platform may have increases service accessibility during the pandemic to a certain extent. However, the well intentioned action to support women victims may cause more harm to them if it not combined with proper precaution.

This presentation aims to provide discussion on the existing challenges in addressing domestic violence, particularly in primary care settings. The arising challenges due to the shift of service delivery due to COVID-19 pandemic are also included. The principles and specific approaches to support victims of violence within the capacity of the organization are elaborated with the intention that clinicians and health administrators can adopt measures that can improve the support for women victims of domestic violence.

\section{Symposium Abstract 27}

Ravishing Face, Heavenly Body: Introduction to Aesthetic Medicine

\section{Adibah HI ${ }^{1}$ \\ ${ }^{1}$ Faculty of Medicine and Health Sciences, Universiti Putra Malaysia}

Aesthetics is a philosophical field that studies the nature of art and our reactions to it. It explores our emotional reactions to natural objects as expressed through the language of beauty and its unattractiveness. Since ancient times, philosophers have debated and argued the concept of beauty. The golden phi, rule of thirds, and rule of five are the mathematical formulas proposed by philosophers for determining facial beauty. They believed that beautiful faces have similar morphological characteristics despite the wide variation in facial types and patterns. However, these characteristics may not be universally applicable across cultures, demographics, and ethnic groups, including Malaysia. Their ethnic identity is essential to them, and they don't want to lose essential face features that signify race.

Women are getting more interested in cosmetic surgery and non-surgical procedures, and this trend is expected to continue. Many people believe that they must look a certain way to be considered beautiful because of the influence of the media and advertising industries. In contrast, many are unaware that the magazine photo has been edited to create an unnatural image. The issues have contributed to the definition of aesthetic medicine in several countries, ensuring a high standard of care for practitioners in the discipline. Licensed medical practitioners are encouraged not to use generic treatment templates without addressing individual characteristics or understanding a patient's idea of beauty, as this could lead to suboptimal outcomes and low satisfaction levels.

Symposium Abstract 28

Men's Health: Issues \& Challenges in Malaysia

\section{Mathews GG1}

${ }^{1}$ Klinik Kesihatan Tampurali, Tuaran, Sabah

Men's Health can be defined as a discipline that promotes the 
physical, mental and social well-being of men throughout their life cycle (from boyhood to manhood), and addresses health problems related to men. Men's health lags behind that of women; men have a shorter life expectancy and higher morbidity and mortality rates compared to women globally, Malaysia included. Malaysian men are traditionally considered as the leader of a household.The reasons for poor health-seeking behaviour among men could be due to male attributes e.g. independence, assertiveness,risk-taking, sense of invulnerability and withstanding of pain, may have contributed to their increased exposure to various health and disease risk factors including hypertension, diabetes and smoking. These roles tend to carry over into health and wellbeing, making men insist on being the leader of their own health, thus posing some 'resistance' to healthcare.

There has been an increasing awareness of men's health in Malaysia over the past decade. Thus, it is timely to encourage men to maintain their own health through provision of health promotion, prevention, treatment and rehabilitation services targeted for men to be further expanded within healthcare facilities and the community. The challenge is to reduce the negative impact that was brought by the image of maleness, and to encourage men to modify their attitudes and behaviours towards themselves and others. It is imperative to establish a friendly health service to men's health to facilitate and provide a place of reference and consultation for specific male health. Strategies through health promotion, accessibility, appropriate health care services, human resource development, men's health information system, inter-sectoral collaboration, research and development including legislation are being placed to empower men to be more proactive in maintaining their own health.

\section{Symposium Abstract 29}

\section{The Needs of Prostate Cancer Survivors in Malaysia}

\section{Lee PY1}

\section{${ }^{1}$ UMeHealth Unit, Faculty of Medicine, University of Malaya.}

In Malaysia, prostate cancer is the third most common cancer among the male population. However, prostate cancer has relatively low mortality rate compared to other cancers. Hence most prostate cancer survivors live with the disease for many years.

We had conducted a qualitative study aimed at exploring the post-treatment impact and needs of prostate cancer survivors. The main issues highlighted by the survivors includes: (a) impact of prostate cancer on the survivors, (b) support needed for coping and (c) information needs. Prostate cancer has an important impact on the survivors' lifestyle and some live with the longterm side effects.

The survivors were anxious about the recurrence of the disease. Family, peers, and spiritual support played an important role in helping them to cope. Most of them felt that their information needs were not being addressed. These information needs includes: post-treatment care, dietary measures and supplements for recurrent prevention, treatment and prognosis. Therefore, it is important for healthcare providers to explore the needs of prostate cancer survivors and provide the necessary support.

Symposium Abstract 30

\section{Providing Inclusive and Holistic Services for the LGBT Community}

\section{Samsul D ${ }^{1}$ \\ ${ }^{1}$ Universiti Islam Antarabangsa Malaysia.}

Health is a state of physical, mental and social well-being, not just the absence of disease or infirmity. This WHO definition was last amended in 1948. We must include spiritual health too. Once we include spiritual health, then gender dysphoria also needs treatment. This community is increasing in trend. Population estimation based on 2018 statistics by Ministry of Health is 15,000 transgenders of Male to Female; Kuala Lumpur is 2,040, Pulau Pinang 1,975, Selangor 1,618. These were the top 3 states. Gay population estimates are 221,698; Kuala Lumpur 53,923, Selangor 50,808, Sabah 19,465. These are the top 3 states. They are vulnerable for HIV.

We have started Integrative medicine: Islamic medicine combined with modern medicine. Modern medicine only focuses on physical illness like HIV, STD and hepatitis. However, the spiritual part is ignored. That is why the transgender community continues doing immoral activities. Our approach is to integrate with Islamic medicine despite us being medical doctors. Our activities include: Iqra' class reciting Al-Quran weekly, outreach programme every 2 months, Hajj and umrah yearly and ibadah camp yearly as well. We also perform mandi jenazah for any LGBT community who passed away. This is the best dakwah. We don't have to say anything. We leave it for the LGBT community to handle from A to Z. It really gives great impact to them, that life is short and temporary.

This is important because from $\mathrm{MOH}$ statistics, homosexuals are increasing in trend. Our treatment so far only focuses on diseases and we miss the spiritual aspect. Pills or tablets can't treat spiritual disorders.

Alhamdulilah, for our achievements: many of the transgenders have started a new life. A few have gotten married. About $60 \%$ of prostitution centres led by them are already closed in Kuantan, Pahang. Our interventions also include non-Malaysians for example from Thailand, Indonesia and etc. So transgenders can be treated and cured, even though the American Psychological Association claimed they can't be.About 10 transgenders have gone breast implant removal surgery which was sponsored by Sultan Ahmad Shah Medical Centre or SASMEC in Kuantan. We also provide them jobs so that they won't relapse to old habits.

Maybe we can't commercialise this activity and we can ' $t$ patent it, but at least we save them from being sex workers. That gives great satisfaction. 
Symposium Abstract 31

\section{Empowering Patient Through social media: Benefits and Challenges}

\section{Suhazeli $\mathbf{A}^{1}$}

${ }^{1}$ Klinik Kesihatan Tengkawang Kuala Berang Hulu Terengganu

Social media is an important tool in delivering your thought since the year 2000. The usage and internet penetration within the last five years has increased tremendously. Total Malaysian internet users in 2020 were 27.1 million. Facebook, Twitter, Instagram are the most hammering tools to influence users' minds. Social media is a sword of two edges. The community will get benefits from the posting if good vibes were promoted or it can also go the other way around. The benefit of social media to patient's care includes engaging users in public health and policy discussions, establishing national and international professional networks, facilitating patients' access to information about health and services, building professional network, sharing ideas and information, offer access to professional and peer support, campaign on issues important to the communities, participates in debates and updates developments around your work. Social media use by patients does not only provide beneficial effects. It may also constitute a challenge within the healthcare system to both patients and healthcare professionals. Since everyone has access to social media, users can post their advice on how to deal with their health condition, thus it is crucial to create a reliable online communication channel to prevent health problems from being exaggerated. Some of the negative effect of social media among medical practitioners includes diminished subjective well-being, loss of privacy, being targeted for promotion and addiction to social media. Many healthcare professionals fear that social media use by patients for health-related purposes often spreads misinformation. This paper will discuss further on the current medical issues in Malaysia and ways to solve them.

\section{Symposium Abstract 32}

\section{Social Media Addiction: Its Impact \& Intervention}

\section{Anne $\mathbf{Y}^{1}$ \\ ${ }^{1}$ Department of Psychological Medicine, University of Malaya Center for Addiction Sciences (UMCAS), Faculty of Medicine, University of Malaya}

Social media addiction is a form of Internet addiction, where individuals exhibit a compulsion to use social media to excess. Excessive social media usage can be described as a type of behavioural addiction as it involves the core components of salience, tolerance, mood modification, withdrawal, conflict, and relapse. Social networking has been shown to be the most highly ranked Internet activity by Malaysian. Studies previously done in Malaysia studying Facebook addiction using the Bergen Facebook Addiction Scale have shown that up to $47 \%$ of tertiary students are addicted to Facebook. The amount of time spent on social media has been positively associated with social media addiction. Many studies on social media usage and mental health have shown that the prolonged use of social media is positively associated with mental health problems such as stress, anxiety, and depression. Treatment option for social media addiction focuses on moderation and controlled use of the Internet rather than total abstinence, and strategies to address the underlying mental health issues.

\section{Symposium Abstract 33}

\section{Engaging Individuals to Undergo CV risk Screening:} What Are the Strategies?

\section{Cheong AT ${ }^{1}$ \\ ${ }^{1}$ Department of Family Medicine, Faculty of Medicine and Health Sciences, Universiti Putra Malaysia}

Cardiovascular disease (CVD) is the leading cause of mortality globally and in Malaysia. The prevalence of cardiovascular (CV) risk factors is high in this country, and more than half of those with $\mathrm{CV}$ risk factors remain ignorant of their cardiovascular risk status. Thus, there is a need to improve the public's participation in CV risk screening for early identification of individuals at high risk for CVD prevention. Literature had showed that individuals' decision to undergo CV risks screening was multi-factorial. Locally, a mixmethod study has provided an understanding of the factors influencing the public's decision to undergo CV risk screening from both individual and general public perspectives. At the individual level, the decision to undergo $\mathrm{CV}$ risk screening was multi-factorial. The main factor was an individual's intention to undergo health checks, which was a result of two key internal factors: the perception of relevance and the state of readiness to act on or cope with the findings of the health checks. The intention of the health checks is subsequently modified by external factors such as influences from significant others, as well as time, cost, accessibility and health care facilities. At the population level, four significant determinants were found to be associated with the intention to undergo $\mathrm{CV}$ risk screening: the perception of benefits and drawbacks of health checks, the perception of external barriers and the readiness to handle outcomes following health checks. These factors can thus be incorporated in developing interventions using effective evidence-based strategies for cardiovascular risk factor screening.

\section{Symposium Abstract 34}

Complementary and Alternative Medicine Use in primary care setting

\section{Ching SM $^{1}$}

${ }^{1}$ Faculty of Medicine and Health Science, Universiti Putra Malaysia

In Malaysia, this is important for primary care doctors to know about complementary alternative medicine (CAM) use among patients as $70 \%$ of patients see their physician before or concurrently having visits to a CAM provider. 
Two third (66.7\%) of patients with stroke used CAM. Among diabetic patients in Malaysia, $62.5 \%$ of them used CAM. Among Chronic kidney disease patients, prevalence of CAM use was $40.3 \%$. Among asthmatic patients, most $(61.1 \%)$ had tried CAM at least once, with $47.4 \%$ still using CAM.

The main types of CAM used among patients with diabetes include herbal and dietary supplements (80\%); reflexology (15\%) and massage (12\%). Bitter gourd, misai kuching, garlic, Sabah snake grass, basil leaf and ginseng were the common herbal products used in Malaysia. Muslim religion predicted CAM use among diabetic patients in Malaysia. Being female and Malays were the determinants of CAM use among patients with asthma.

More than three quarters $(78.1 \%)$ of CAM products were unregistered under Malaysian Regulatory Authority, 16.7\% of which contained mainly dexamethasone. Routine use of natural products among asthmatic patients could cause poor adherence rate, which lead to poorer asthma control and higher hospitalization rate.

Physicians never ask their patient on CAM use is the main barrier for patients to disclose CAM use to healthcare providers. The solution is to ASK our patient about CAM use during history in a non-judgmental way and apply shared decision-making strategy to help patients to gain potential benefit and avoid harm.

\section{Symposium Abstract 35}

Preventing Diabetes in the Offspring: What does it take?

\section{L Whitford ${ }^{1}$}

\section{${ }^{1}$ RCSI \& UCD Malaysia Campus, Pulau Pinang, Malaysia}

Background: The rising prevalence of type 2 diabetes (T2DM) led to a multitude of randomised controlled trials indicating it was possible to prevent or reduce progression to T2DM in high-risk individuals. Initial trials were carried out in individuals with the highest risk of diabetes, namely those with pre-diabetes. These involved population screening to identify pre-diabetes and intensive lifestyle interventions. This raised doubts as to whether this approach was costeffective or pragmatic. Simpler methods of identifying high risk individuals and intervening in lifestyle have since been proposed. First-degree relatives of people with T2DM are at increased risk of developing this condition, with offspring and siblings at a three-fold higher risk than the general population. Identifying and intervening in this group could therefore form part of an effective T2DM prevention strategy. Method: This is a review of the research on preventing diabetes in offspring of people with T2DM.

Results: There has been limited research evaluating interventions that target modifiable risk factors for T2DM in relatives of people with T2DM. However, the majority of studies demonstrate a willingness of patients with T2DM and their offspring to be informed of their diabetes risk. The small number of intervention trials to date indicate the effectiveness of intervening in relatives of patients with T2DM to prevent diabetes.
Discussion: There are two main challenges to optimize diabetes prevention in high-risk relatives of people with T2DM: the development of effective interventions that are sustainable and not demanding on participants' time and cost; and simple and feasible methods of recruiting people who would benefit most from them. I propose a potential solution to these challenges.

\section{Symposium Abstract 36}

Managing chronic Hepatitis C in primary care : a reality in Kedah

\section{Habshoh $\mathbf{H}^{\mathbf{1}}$ \\ ${ }^{1}$ Klinik kesihatan Bandar Sungai Petani, Kedah}

Hepatitis C Virus causes acute and chronic hepatitis infection. It has a wide spectrum of illness from mild to severe debilitating illness. Cure for chronic hepatitis C infection can be achieved with a new drug called DAAs Directly Acting Antivirals. A new strategy to achieve WHO vision to eliminate hepatitis by 2030 includes making diagnosis and treatment accessible to all. As primary care service is distributed well in all states and districts, it is accessible to all and nearer to clients. Simplifying method of diagnosis and treatment and making it available at Primary care service is the best way forward to achieve the goal.

Kedah state started the challenge in treating chronic HCV in primary care in 2018 successfully. It was started with two health clinics and by 2019 all health clinic with resident Family Medicine Specialist can treat chronic hepatitis C. Besides health clinic, medical team in Prison were also trained and can start treatment for prisoners. Person aged 18 and above, treatment naïve, non-cirrhotic and compensated cirrhosis as well as co-infection with HIV can be treated at primary care for a duration of 12 to 24 weeks. Treating chronic HCV at all health clinics in Malaysia will make our vision to eliminate hepatis C by 2030 a reality.

\section{Symposium Abstract 37 \\ Influenza: Forget Me not}

\section{Petrick $\mathbf{P}^{1}$ \\ ${ }^{1}$ Faculty of Medicine, Hospital UKM}

Influenza is an envelope virus which is divided into 3 types, 2 subtypes and 2 lineages. So far Type A has caused many pandemics. It affects 3-5 million people in the world and causes up to 0.65 million deaths per year. In those aged 75 and above the mortality can go up to 51.3-99.4 per 100,000 individuals. Malaysia influenza rates can range from ranging from $7-12 \%$.

The average incubation period is 1-4 days. Differentiating it from cold and flu and covid can be very challenging. Most uncomplicated influenza resolves in a week. But is minority of patient's, systemic secondary complications can occur. Infected individual can become infectious 1 day before symptoms and up to 5 to 7 days after falling ill. 
Influenza virus can remain viable on surfaces for some time. The rate of transmission from an asymptomatic person is usually $1 / 3$ the rate of transmission from symptomatic individuals.

PCR test has the best sensitivity compared to rapid antigen testing .

Non pharmacological management is just as important as pharmacological management .Treatment is essential especially in the high risk groups. Oral oseltamivir which has been the mainstay drug for some time is efficacious. Newer drugs like Oral Baloxavir improves compliance with a single dose option and rapidity of its action .

Finally, prevention is better than cure, high risk groups should be offered vaccination.

\section{Symposium Abstract 38}

\section{COVID-19 vaccination: New updates}

\section{Strain ${ }^{1}$ \\ ${ }^{1}$ University of Exeter, $U K$}

The ongoing COVID-19 pandemic, caused by severe acute respiratory syndrome coronavirus 2 (SARSCoV-2), has resulted in more than 193 million confirmed cases of COVID-19 and more than 4 million deaths globally as of 26 July 2021. Vaccinations against SARS-CoV-2 reduce serious illness and transmission, thereby limiting the health and socioeconomic impacts of the pandemic. The global rollout of vaccines to adults is proceeding apace, with the World Health Organisation (WHO) reporting that 3.6 billion vaccine doses have been administered as of 25 July 2021. The success of vaccination programmes depend upon multiple factors including vaccine efficacy, speed of vaccine rollout and uptake rates, emergence of new variants, patterns of social mixing and, critically, population vaccination strategies. In countries where an increasing percentage of adults are fully vaccinated, but where children and adolescents are not, debate persists as to the population relevance of the high prevalence of SARS-CoV2 in children and adolescents and the role of vaccinating younger age groups in controlling the pandemic. This presentation will discuss the different vaccine strategies, the pitfalls and advantages of the different vaccines and the current controversies.

\section{ORAL ABSTRACTS}

\section{Oral Abstract 1}

A Feasibility Study of a School-Based Asthma Programme in Malaysia Conducted During COVID-19 Pandemic

Ramdzan $\mathrm{SN}^{1,2}$, Khoo $\mathrm{EM}^{1}$, Liew $\mathrm{SM}^{1}$, Cunningham $\mathrm{S}^{2,}$

Pinnock $\mathrm{H}^{2}$

${ }^{1}$ Department of Primary Care Medicine, Faculty of Medicine, University of Malaya, Malaysia; ${ }^{2}$ NIHR Global Health Research Unit on Respiratory Health (RESPIRE), Usher Institute,

University of Edinburgh, United Kingdom

Introduction: To assess the feasibility of a school-based asthma programme adapted to comply with COVID-19 restrictions. The programme aimed to improve control of children with asthma.

Methods: The study was conducted in October 2020 in a school in Malaysia. We identified children with asthma using a screening questionnaire. We planned to deliver sessions to school children with asthma and their parents, school staff and all school children, measure the participation rate and feedback on the components of the programme.

Results: We received a good response rate $(74.5 \%)$ and identified 34 (3.7\%) children with asthma who were eligible to receive the intervention. The session for school staff received good participation $(88.7 \%)$ and feedback $(>80 \%$ scored good/excellent). In mid-October, COVID-19 forced national school closure disrupting the study. Sessions for children with asthma were conducted remotely, and sessions for other school children were abandoned. Only 14/34 (41.2\%) children with asthma attended the remote sessions in December 2020. Parents were either uncontactable (9/34: $26.5 \%)$ or declined participation (11/34: 32.3\%) because they lacked internet facilities or timing was inconvenient (albeit sessions were out-of-hours). Children liked the sessions as they were interactive and used various methods, e.g., videos, games to deliver messages. Parental participation was minimal $(<34 \%)$ because of other commitments.

Conclusions: The school-based intervention was feasible, though restricted by the pandemic. Although remote health programmes gained popularity during the pandemic, widespread use could disadvantage children from lower socioeconomic backgrounds who had limited access to the internet and electronic devices and may not be a long-term equitable solution.

\section{Oral Abstract 2}

\section{Factors Associated With Sexually Transmitted Infections (STI) and STI Rates at Teluk Intan Health Clinic (KKTI) from 2014 to 2018}

\section{Suriata $\mathbf{D}^{1}$ \\ ${ }^{1}$ Klinik Kesihatan Teluk Intan, Jalan Bandar 36000 Teluk Intan, Perak.}

Introduction: STI client friendly clinic in KKTI was started in 2014 with focus on high-risk groups in the Hilir Perak District. The purpose of this study was to evaluate the frequency of STI diagnoses and the trend of STI rates in this clinic from 2014 to 2018.

Methods: This retrospective analysis included consultations between 2014 to 2018. The inclusion criteria for this study were patients 18 years and older who acquired care for STI during the study period. The collection sheet included patient's age, gender, date of consultation, ethnicity, previous STI, symptoms, current STI diagnoses, treatment and highrisk behavior assessment. Data were transferred to a spread sheet using SPSS Version 23. Chi-square tests were used to analyze the STI trend and to find significant associations between patients' factors and STI diagnosis. P value $<0.05$ was set as significant. 
Results: The number of consultations was 193. Several factors were found to be significantly associated with STI. The overall STI positive rate was $18.7 \%$. The trend for syphilis, gonorrhoea and HIV were stable throughout the study period ( $x^{2}$ for trend, $\mathrm{p}=0.12$ ).

Conclusion: The effectiveness of STI preventive programs in this community need to be reviewed because this study showed stable rates of STI diagnoses over the study period.

\section{Oral Abstract 3}

Factors Influencing the Practice of Smoking Cessation Assessment and Management Among Primary Health Care Doctors in Selangor Primary Healthcare Clinics (SCAAM DOC)

\author{
Ngee L J B ${ }^{1}$, AT Cheong ${ }^{1}$, Abdul Hadi AM ${ }^{1}$ \\ ${ }^{1}$ Faculty of Medicine and Health Sciences, Universiti Putra \\ Malaysia, Serdang, Selangor, Malaysia.
}

Introduction: Smoking prevalence remains high in Malaysia. Primary care doctors have good opportunity to motivate smokers to quit smoking in view of the accessibility of primary healthcare clinics to the public. The objective was to determine the level of knowledge, attitude and factors associated with the practice of smoking cessation management among doctors in the pre-contemplation and contemplation phase.

Methods: A cross-sectional survey was done among 383 medical officers and interns in all government healthcare clinics at the district of Petaling, Klang and Hulu Langat. The knowledge, attitude and practice (KAP) of smoking cessation management were assessed using seventeen items validated questionnaire which covered the components of 5As (Ask, Advise, Assess, Assist, Arrange) and 5Rs (Relevance, Risk, Reward, Roadblocks, Repetition).

Results: Majority of the respondents had poor scores of knowledges (62.4\%); attitude (58\%) and practice (Precontemplation:50.9\%; contemplation:75.7\%). Using multivariate logistic regression analysis, the significant factors associated with poor practice of smoking cessation management at precontemplation phase were poor $(\mathrm{OR}=2.143,95 \%$ CI 1.115- 4.120, $\mathrm{p}=0.022)$ or moderate knowledge $(\mathrm{OR}=2.507,95 \%$ CI $1.193-5.268, \mathrm{p}=0.015)$, poor attitude $(\mathrm{OR}=2.169,95 \%$ CI $1.393-3.377, \mathrm{p}=0.001)$, lacks of smoking cessation banners, brochures and leaflets in the clinic $(\mathrm{OR}=2.012,95 \% \mathrm{CI} 1.266-3.198, \mathrm{p}=0.003)$ and lack of nicotine replacement medications $(\mathrm{OR}=2.278$. 95\%CI 1.277-4.064, $\mathrm{p}=0.005$ ). No significant factors were shown associated with the practice in the contemplation phase.

Conclusion: KAP among primary care doctors were suboptimal. Factors that increased odds for smoking management's poor practice in the pre-contemplation phase were poor knowledge, poor attitude, and insufficient organizational support. Interventions need to target these factors to improve doctors' management.
Oral Abstract 4

Intention and Practice on Breastfeeding Among Pregnant Mothers in Kinta District and Factors Associated With Practice of Exclusive Breastfeeding: A Cohort Study

Nurul Mursyidah $\mathbf{S}^{1}$, Majidah $\mathrm{M}^{2}$, Kanesh $\mathrm{R}^{3}$, Mai Shahira $\mathrm{MH}^{4}$, Leong $\mathrm{YN}^{5}$, Cheong XB ${ }^{6}$, Cheong $\mathrm{AT}^{7}$, Subashini $\mathrm{A}^{1}$ ${ }^{1}$ Klinik Kesihatan Buntong, Persiaran Desa Rishah 2, Taman Desa Rishah, 30100 Ipoh, Perak, Malaysia; ${ }^{2}$ Klinik Kesihatan Jelapang, Jalan Jelapang, 30020 Ipoh, Perak, Malaysia Jalan Jelapang, 30020 Ipoh, Perak, Malaysia; ${ }^{3}$ Klinik Kesihatan Tanjong Malim, Jalan Besar, 35900 Tanjong Malim, Perak, Malaysia; ${ }^{4}$ Klinik Kesihatan Pantai Remis, Jalan Besar, Pekan Pantai Remis, 34900 Pantai Remis, Perak, Malaysia; ${ }^{5}$ Klinik Kesihatan Simee, Jalan Kg Simee 31400 Ipoh Perak, Malaysia; ${ }^{6}$ Klinik Kesihatan Chemor, Jalan Tanah Hitam, Kampung Chemor, 31200 Chemor, Perak, Malaysia; ${ }^{7}$ Faculty of Medicine and Health Sciences, Universiti Putra Malaysia, 43400

Serdang, Selangor, Malaysia

Introduction: Exclusive breastfeeding rate in Malaysia is low despite its known health benefits. This study aims to determine the prevalence of intention to breastfeed among pregnant mothers, prevalence of exclusive breastfeeding practice after delivery and factors associated with exclusive breastfeeding practice.

Methods: This was a prospective cohort study. All pregnant women at 36 weeks gestation or above from 17 antenatal health clinics in Kinta, Perak were invited to participate in the study. A self-administered questionnaire was used which encompassed sociodemographic, knowledge, attitude, and intention towards practice of breastfeeding. The participants were follow-up one-month post-natal for their practice of breastfeeding via telephone or during their postnatal follow-up. Results: 483 pregnant mothers participated in the study initially but only $462(95.7 \%)$ were contactable after one month. Participants who intended to breastfeed are 99.4 $\%$ (459/462) while participants who practised exclusive breastfeeding are $65.4 \%(302 / 462)$. There is no significant association between intention and practice of exclusive breastfeeding. Bivariate analysis shows factors that are associated with exclusive breastfeeding includes race $(\mathrm{p}=0.025)$, knowledge $(\mathrm{p}<0.001)$ and attitude $(\mathrm{p}=0.020)$. Using multivariate logistic regression, high knowledge (AOR $=1.138 ; 95 \%$ CI $1.008,1.284)$ and Malay ethnicity $(\mathrm{AOR}=$ 2.031; 95\% CI 1.066, 3.868) were more likely to breastfeed their infant exclusively.

Conclusion: Prevalence of exclusive breastfeeding practice at one month in Kinta district was moderate. Malay mothers and mothers with high knowledge were more likely to exclusively breastfeed. Thus, we recommend targeted intervention towards non-Malay mothers and increasing breastfeeding knowledge to all pregnant mothers. 
Oral Abstract 5

The Accuracy of Detecting Diabetic Retinopathy (DR) Using Panoptic Ophthalmoscope (PO) by Primary Care Doctors at Klinik Kesihatan (KK) Sendayan, Negeri Sembilan

Nur Aishah $\mathbf{A L}^{1}$, Mastura $\mathrm{I}^{2}$, Maznun $\mathrm{MJ}^{3}$, Nor Nadiah $\mathrm{I}^{1}$, Mohd Amin $\mathrm{F}^{1}$

${ }^{1}$ Klinik Kesihatan Sendayan, Negeri Sembilan; ${ }^{2}$ Klinik Kesihatan Seremban2, Negeri Sembilan; ${ }^{3}$ Klinik Kesihatan Triang, Pahang

Introduction: DR is known to cause blindness. Early detection is vital to prevent further deterioration. However, fundus camera as the gold standard tool for DR screening, is not widely available in primary care. This study was done to determine the accuracy of DR detection using panoptic ophthalmoscope (PO) by the primary care doctors.

Methods: 66 diabetic patients were enrolled in this observational study. Systematic randomized sampling was applied. Pan ophthalmoscopy and fundus photography were done on the same day for DR detection. Medical officers (MO) who did PO would interpret their findings and decide whether require referral or not. Fundus photography was interpreted by an ophthalmologist as the gold standard. Both findings and decisionsfor referral were compared and analyzed. Results: PO by MO showed only 53\% accuracy in detecting DR. The sensitivity and specificity of DR detection using PO was $10.3 \%$ and $86.5 \%$ respectively. The accuracy of sight threatening abnormalities (STA) detection was 75.8\%. The sensitivity and specificity of STA was $35.3 \%$ and $89.8 \%$, respectively. The proportion of newly diagnosed DR was $43.9 \%$, while the proportion of STA was $25.8 \%$. DR was significantly associated with patients living with diabetes for more than 10 years $(\mathrm{p}=0.007)$.

Conclusion: The accuracy of PO in DR screening was poor, but it was modestly accurate for STA detection. However, the sensitivity was low, hence DR and STA might be missed during screening in primary care. We recommend the availability of fundus camera in every primary care setting.

\section{Oral Abstract 6}

Mass-media intervention to improve breast cancer awareness and screening among women in Malaysia

Mila NNH ${ }^{1,2}$, Maznah $\mathrm{D}^{1,3}$, D Schliemann ${ }^{4}$, Siew $\mathrm{YL}^{5}$, Saunthari $\mathrm{S}^{6}$, Nor Saleha TI ${ }^{7}$, M Donnelly ${ }^{4}$, Tin TS ${ }^{1,8}$ ${ }^{1}$ Centre for Population Health (CePH), Department of Social and Preventive Medicine, Faculty of Medicine, University of Malaya, Kuala Lumpur, Malaysia; ${ }^{2}$ Department of Community Medicine, Melaka-Manipal Medical College, Manipal Academy of Higher Education (MAHE), Melaka, Malaysia; ${ }^{3}$ Department of Health Policy and Management, Faculty of Public Health, Universitas Airlangga, Surabaya, Indonesia; ${ }^{4}$ Centre for Public Health and UKCRC Centre of Excellence for Public Health, Queen's University Belfast, Belfast, UK; ${ }^{5}$ Department of Rehabilitation Medicine, University of Malaya, Malaysia; ${ }^{6}$ National Cancer Society, Kuala Lumpur, Malaysia; ${ }^{7}$ Ministry of Health, Putrajaya, Malaysia 8 South East Asia Community Observatory (SEACO), Monash University Malaysia, Bandar Sunway, Malaysia

Introduction: Delayed presentation of breast cancer (BC) often leads to increased morbidity and poor survival rates amongst Malaysian women. This study designed, implemented, and evaluated a breast cancer awareness raising mass-media campaign ("Be Cancer Alert Campaign" BCAC-BC) to enhance awareness and screening.

Methods: The BCAC-BC was conducted for 5 weeks through TV, radio, and print media. A pre-post intervention study was conducted in Petaling district. Women aged 40 years and older from randomly sampled households were surveyed between June 2018 and January 2019. Breast cancer screening data was collected from the primary healthcare clinics and three public hospitals.

Results: A total of 676 respondents completed the pre- and post-survey, and $65 \%$ of respondents reported that they saw the BCAC-BC materials. Participants who noticed at least one of the BCAC-BC materials showed significant improvements in non-lump symptom awareness $(71 \%$ vs $81 \%)$, breast selfexamination (BSE) awareness (48\% vs 66\%) and confidence to notice a BC symptom (65\% vs $73 \%)$ post-campaign. The mammogram uptake improved by $16 \%$ and $\mathrm{BC}$ diagnosis increased by $13 \%$ compared to the pre-BCAC-BC implementation period.

Conclusion: The BCAC campaign was associated with improved awareness about $\mathrm{BC}$ symptoms and awareness to BSE among Malaysian women. Further efforts are required to translate increased BC awareness into screening uptake. There may be a need to give attention to ways in which to support healthcare providers to be proactive in terms of initiating discussion about breast health and offering CBE to women who attend their clinics, especially in the primary healthcare sector.

\section{Oral Abstract 7}

A cross-sectional study on the level of mindful eating among Type 2 Diabetes Mellitus patients and its associated factors

\section{Nurul Hayati $\mathbf{C}^{\mathbf{1}}$, Noorlaili $\mathrm{MT}^{2}$ \\ ${ }^{1}$ Department of Primary care, Faculty of Medicine and Health Sciences, Universiti Sains Islam Malaysia (USIM), 71800, Nilai, Negeri Sembilan, Malaysia; ${ }^{2}$ Department of Family Medicine, Faculty of Medicine, Universiti Kebangsaan Malaysia, 43600, Bangi, Selangor, Malaysia}

Introduction: Mindful eating is self-regulation of attention towards food and eating experience in a non-judgmental manner. Its benefits in initiating and maintaining healthy eating behaviour were being studied. The studies addressing mindful eating among Type 2 Diabetes Mellitus patients (T2DM) are limited; hence, this study aims to determine the level of mindful eating using the mindful eating questionnaire (MEQ) among T2DM patients at Klinik Kesihatan (KK) Kajang and its associated sociodemographic factors and clinical profile. 
Methods: A cross-sectional study was conducted among adult T2DM patients attending diabetic follow-up at KK Kajang. Respondents were selected via systematic random sampling. Mindful eating level were assessed using a self-administered questionnaire (MEQ). MEQ mean score ranges from one to four, with a higher mean score indicating a higher mindful eating level.

Results: Two hundreds respondents participated with mean age of $57 \pm 11$ years old. The mean of mindful eating was $2.90 \pm 0.25$. Older age $\left(\mathrm{AOR}^{*}=1.05, \mathrm{p}=0.01\right)$ and fasting blood sugar $>7 \mathrm{mmol} / \mathrm{L} \quad(\mathrm{AOR}=2.55, \mathrm{p}=0.01)$ were independently associated with high mindful eating level.

Conclusion: The level of mindful eating among T2DM patients was relatively higher than obese people, albeit lower than pregnant women and yoga practitioners across the world. Mindful eating could be an option in managing T2DM patients at primary care, particularly among young patients. FBS level could be used to motivate patients to be more aware of their eating behaviour as the first step in advocating mindful eating.

\section{Oral Abstract 8}

Baby walker usage among toddlers in Tangkak Johor: have we done enough?

\author{
Mohd Hazizul AS ${ }^{1,2}$, Hizlinda $\mathrm{T}^{2}$, Rosdina $\mathrm{AK}^{1}$, \\ Noor Azimah $\mathrm{M}^{2}$ \\ ${ }^{1}$ Sungai Mati Health Clinic, 84400 Tangkak, Johor; \\ ${ }^{2}$ Department of Family Medicine, Faculty of Medicine UKM, \\ 56000 Cheras, Kuala Lumpur
}

Introduction: Baby walkers have been used for many centuries and still widely accepted even with recommendations against its use. This study aimed to determine the prevalence of its use and describe injuries associated, and advice received from health care providers (HCP).

Methods: This is a cross-sectional study involving parents of children aged 6 to 15 months attending primary care clinics in Tangkak, Johor using a validated questionnaire assessing baby walker's use, history of related injuries and advice recieved from healthcare providers (HCP).

Results: The mean (SD) age of parents was 31.9 (5.2) years with a majority of them females $(97.1 \%)$ and married (95.6\%). The prevalence of baby walker use was $76.8 \%$. The median (IQR) duration of use was 30 (15) minutes of 3 (2) times daily. Almost all the baby walker use was supervised and only $9.2 \%$ reported injuries involving head, face, limbs, and trunk injuries. Only $30.7 \%$ of the parents reported they received advice from HCP that include against its use, dangers associated with risks and the non-proven benefits in terms of child's growth and development. However, parents also received advice from HCP on how to use it safely.

Conclusion: The use of baby walker among toddlers in Tangkak was still prevalent and a substantial proportion of the toddlers experienced physical injuries. Less than a third of the parents received advice on baby walkers and some were wrongly advised. There is a need to educate HCPs on its use, along with public education programmes on its dangers.

\section{Oral Abstract 9}

Knowledge and attitude on childhood vaccination among healthcare workers in Hospital Universiti Sains Malaysia (HUSM)

\author{
Ahmad Faiq $\mathbf{M}^{1}$, Azidah $\mathrm{AK}^{1}$, Norhayati $\mathrm{MN}^{1}$ \\ ${ }^{1}$ Department of Family Medicine, Kampus Kesihatan, Universiti \\ Sains Malaysia, 16150 Kubang Kerian, Kota Bharu, Kelantan
}

Introduction: Good knowledge and attitudes on childhood vaccination among healthcare workers $(\mathrm{HCW})$ are important as they are the most trusted source of vaccine-related information for patients. This study was conducted to assess the knowledge and attitudes on childhood vaccinations among HCW in Hospital Universiti Sains Malaysia (HUSM) and associated factors for decision-making capable of positively affecting the vaccination practice among children.

Methods: A descriptive, cross-sectional study involving 198 participants was conducted in HUSM with convenient sampling technique. A validated, self-administered questionnaire was used to collect the data from eligible participants. Descriptive and logistic regression analyses were used to express the results.

Results: A total of $68.7 \%$ of participants exhibited good knowledge about childhood vaccination. Female gender $(\mathrm{p}<0.05)$ and higher education $(\mathrm{p}<0.05)$ were significantly associated with good knowledge of childhood vaccination whereas participants with a history of side effects of vaccine among their relatives were about $66 \%$ less likely to have good knowledge $(\mathrm{p}<0.05)$. A total of $59.1 \%$ of participants displayed good attitudes towards childhood vaccination. Higher education level $(\mathrm{p}<0.001)$ was significantly associated with good attitudes. Participants having direct contact with patients $(\mathrm{p}<0.05)$ and a history of severe side effects of vaccine among their relatives $(\mathrm{p}<0.05)$ were likely to have a poor attitude.

Conclusion: The study showed good knowledge and attitudes of participants towards childhood vaccination. Direct contact with patients and history of severe side effects of vaccine were reported by the participants as the barriers towards childhood vaccination.

\section{Oral Abstract 10 \\ Internet addiction among early adolescents during COVID-19 pandemic in the east coast of Peninsular Malaysia}

Lena NS ${ }^{1,2}$, Azidah AK ${ }^{1,2}$, Lili Husniati $\mathrm{Y}^{1,2}$, Faridah $\mathrm{MZ}^{3}$
${ }^{1}$ Department of Family Medicine, School of Medical Sciences,
Universiti Sains Malaysia, 16150, Kubang Kerian, Kelantan,
Malaysia; ${ }^{2}$ Hospital Universiti Sains Malaysia, Jalan Raja
Perempuan Zainab II, 16150, Kubang Kerian, Kelantan,
Malaysia; ${ }^{3}$ MSU Medical Centre, Jalan Boling Padang 13/64,
Seksyen 13, 40100, Shah Alam, Selangor, Malaysia

Introduction: Internet addiction among early adolescents during COVID-19 pandemic in the east coast of Peninsular Malaysia. The study aimed to determine the prevalence of 
Internet addiction (IA) and identify its associated factors among early adolescents.

Methods: A cross-sectional online survey was conducted from February 2021 to April 2021 among adolescents aged 13 to 14 years old in the east coast of Peninsular Malaysia. Participants completed a series of a questionnaire containing Malay Version Internet Addiction Test (MVIAT), Depression, Anxiety and Stress Scale- 21 (DASS-21) and psycho sociodemographic information according to Malaysian Global School-based Student's Health Survey. Multiple logistic regression analysis was done to explore the associations between the variables.

Results: A total of 535 participants enrolled with a preponderance of female $(65.9 \%)$. Based on the optimal cut-off for MVIAT (43/100), the prevalence of IA among participants are $51.4 \%$. The significant factors associated with increased risk of IA were boys $(\mathrm{AOR}=2.15,95 \% \mathrm{CI}$ $=1.30-3.57)$, internet usage at home $(\mathrm{AOR}=6.17,95 \%$ $\mathrm{CI}=1.94-19.58)$, using internet to watch/download music $(\mathrm{AOR}=2.50,95 \% \mathrm{CI}=1.52-4.12)$, social networking $(\mathrm{AOR}=2.29,95 \% \mathrm{CI}=1.19-4.40)$, smokers $(\mathrm{AOR}=$ 12.39, 95\% CI $=1.34-114.43)$, depression $(\mathrm{AOR}=2.03$ , $95 \% \mathrm{CI}=1.07-3.85)$, anxiety $(\mathrm{AOR}=2.79,95 \% \mathrm{CI}$ $=1.64-4.76)$, and stress $(\mathrm{AOR}=4.67,95 \% \mathrm{CI}=2.00-$ 10.91).

Conclusion: Internet addiction is prevalent among Malaysian adolescents during the COVID-19 outbreak. Sex, internet use profile, and adolescent psychological factors were significant predictors for IA.

\section{Oral Abstract 11}

Prevalence of falls and their associated factors among the hypertensive elderly in East Coast Malaysia

Atirah Az-Zahra' AB ${ }^{1}$, Azidah $\mathrm{AK}^{1}$, Nur Suhaila $\mathrm{I}^{1}$, Siti Nurbaya $\mathrm{MN}^{2}$

${ }^{1}$ Department of Family Medicine, School of Medical Sciences, Universiti Sains Malaysia, Kubang Kerian, Kelantan, Malaysia; ${ }^{2}$ Department of Internal Medicine, School of Medical Sciences, Universiti Sains Malaysia, Kubang Kerian, Kelantan, Malaysia

Introduction: Falls are prominent health issues among the elderly. Among the hypertensive elderly, falls may have a detrimental effect on their health and wellbeing. The purpose of this study is to determine the prevalence of falls among the hypertensive elderly and to identify the associated factors that contribute to their falls.

Methods: This was a cross-sectional study conducted in two health clinics in Kuala Terengganu, Malaysia. Data on their socio-demographic details, their history of falls, medication history, and clinical characteristics was collected. Balance and gait were assessed using the Performance Oriented Mobility Assessment (POMA).

Results: Two hundred and sixty-nine elderly with hypertension were recruited. It was found that $32.2 \%$ of participants reported falls within a year. Polypharmacy (OR $2.513,95 \%$ CI 1.339, 4.718) and diuretics (OR 2.803, 95\% CI $1.418,5.544)$ were associated with an increased risk of falls. Meanwhile, a higher Performance Oriented Mobility Assessment (POMA) score (OR 0.940, 95\% CI 0.886, 0.996 ) and a number of antihypertensives (OR 0.473, 95\% CI $0.319,0.700)$ were associated with a reduced risk of falling among the hypertensive elderly.

Conclusion: Falls are common among the hypertensive elderly. Elderly patients who are taking diuretics and have a polypharmacy treatment plan have a higher risk of falls. However, the elderly taking a higher number of antihypertensive medications specifically were not associated with an increased risk of falls.

\section{Oral Abstract 12}

The interest and willingness to pay for a suitable Takaful products among diabetes patients in Malaysia. a nationwide descriptive analysis in Malaysia

Mohd Radzniwan AR ${ }^{1}$, Azrul Azlan $\mathrm{IM}^{2}$, Nurul Aini $\mathrm{M}^{2}$, Zurina K@Z $Z^{2}$, Junaidah $\mathrm{AS}^{2}$, Aimi Fadzirul $\mathrm{K}^{2}$, Sharifah Fairuz $\mathrm{SM}^{3}, \mathrm{SA}^{4}$

${ }^{1}$ Faculty of Medicine and Health Science, Universiti Sains Islam Malaysia, Malaysia; ${ }^{2}$ Faculty of Economics and Muamalat, Universiti Sains Islam Malaysia, Malaysia; ${ }^{3}$ Faculty of Science and Technology, Universiti Sains Islam Malaysia, Malaysia; ${ }^{4}$ Universiti Kebangsaan Malaysia Medical Centre, Universiti Kebangsaan Malaysia, Malaysia.

Introduction: The cost of diabetes management is exorbitant. The insurance option for the diabetic is limited and there is no takaful product offered. The study aimed at determining the interest and willingness to pay (WTP) for takaful products among diabetes patients in Malaysia.

Methods: A nationwide multicentred cross-sectional random sampling study was conducted from December 2020 to March 2021 among adults' type 2 diabetes patients whereby type 1 diabetes was excluded. Self-administered questionaries were used to obtain data on clinical profiles and assess the interest and WTP for takaful products. Ethical approval was obtained. Data were analyzed descriptively.

Results: Five hundred participants were enrolled. The majority have the mean diabetes duration of 9.3 years (SD 7.08) with two-third (67.4\%) had poor glycemic control (HbA1c > 7\%) and 53.9\% have no diabetes complications. With regards to the interest, $60.7 \%$ indicated their interest in takaful products especially among Malay (45\%), B40 groups $(47.5 \%)$, pension/retire (19\%) and those of secondary education background (32\%). Twenty-eight percent were still unsure with $11.2 \%$ were not interested. With regards to WTP, the majority (74\%) preferred the lowest payment (< RM100), the monthly payment (71\%), and chose to pay through a bank $(74 \%)$. The top three beneficial they need were medication (81.8\%), counselling (63\%) and glucometer (55.8\%).

Conclusion: The majority of diabetics in this study were interested in takaful products for health protection but they were willing to pay minimally. Proper understanding of the need is crucial in developing suitable takaful products for diabetes patients in Malaysia. 
Oral Abstract 13

A Focus on Religious and Spiritual Coping Among Malay Muslim MSMS With HIV in Malaysia: A Qualitative Study

Aiza Nur Izdihar ZA ${ }^{1}$, Farnaza $\mathbf{A}^{\mathbf{1 , 3}}$, Siti Fatimah BS ${ }^{1}$, Salmi $\mathrm{R}^{2,3}$ ${ }^{1}$ Primary Care Medicine, Faculty of Medicine, Universiti Teknologi MARA, Sungai Buloh Campus, Selangor; ${ }^{2}$ Psychiatry and Behavioural Medicine, Faculty of Medicine, Universiti Teknologi MARA, Puncak Alam Campus, Selangor; ${ }^{3}$ Maternofoetal and Embryology Research Group (MatE), Faculty of Medicine, Universiti Teknologi MARA, Sungai Buloh Campus, Selangor

Introduction: The increase in life expectancy of PLHIV has brought new challenges especially for young Malay Muslim men who have sex with men (MSM) in Malaysia. This country has strong religious and cultural roots that may pose as additional stigma and discrimination in the lives of PLHIV. Coping skills among PLHIV is important and we are interested to explore religious and spiritual coping among this population.

Methods: This was a qualitative, in-depth interview study. It recruited eligible participants using purposive and snowballing sampling techniques via an NGO and online flyers. The narrative inquiry approach was used to understand the life story comprehensively. The interviews were transcribed verbatim and analysed using Qualitative Data Analysis (QDA) Miner. It was analysed using a phenomenological approach to create meaning behind their lived experience.

Results: Interviews with seven participants yielded four themes. Ambivalence towards religion was experienced by participants at some point following their diagnosis. Using religion/spirituality as a form of self-reflection was identified. Having positive religious support from family or NGOs helped some participants to embrace religious coping rather than to avoid it, and contemplation and relationship with God was an important aspect of coping.

Conclusion: Previous studies supported the search for the spiritual meaning of illness as a coping strategy for PLHIV. This study suggests the need for spiritual and religious guidance as well as positive support from healthcare professionals and religious-based organizations for PLHIV, for them to embrace religious and spiritual coping to handle the disease and improve health outcomes.

\section{Oral Abstract 14}

Home medication management and its associated factors among long term stroke patient in a community based primary care clinic

Mohd Hafizul AN ${ }^{1}$, Noor Azah $\mathrm{AA}^{1}$, Isa $\mathrm{NM}^{2}$,

Mohd Makmor B ${ }^{3}$, Mohd Fairuz $\mathrm{A}^{1}$

${ }^{1}$ Department of Family Medicine, Faculty of Medicine, Universiti Kebangsaan Malaysia, 43600, Bangi, Selangor, Malaysia; 'Department of Pharmacology, Faculty of Medicine, Universiti Kebangsaan Malaysia, 43600, Bangi, Selangor, Malaysia; ${ }^{3}$ Faculty of Pharmacy, Universiti Kebangsaan Malaysia, 43600, Bangi, Selangor, Malaysia
Introduction: Stroke survivors often have multiple and complex needs, coupled with physical disabilities, presence of polypharmacy, risk of adverse drug reactions and recurrent stroke. This could have an impact on the medication management process. This study aimed to determine home medication management practices (MMP) and its associated factors.

Methods: This cross-sectional, single-center study involved 120 stroke patients attending a community based primary care clinic in Kuala Lumpur between January and March 2021. Appropriateness of MMP was assessed through scores of questions using face to face interview, telephone interview and Google form.

Results: The mean age of participants was 65.1 (SD 11.7) years. The prevalence of inappropriate home MMP was $55.8 \%$. Most participants $(86.3 \%)$ did not update their medication list, $75 \%$ did not memorize the medication's name and $63.3 \%$ did not return the leftover medication to the pharmacy. In the multivariate model, independent variables significantly associated with appropriate home MMP were female gender (AOR: 2.74, 95\% CI: 1.18-6.35, p-value: 0.019), household income (> RM4850) (AOR: 2.82, 95\% CI: 1.03-7.69, p-value: 0.043$)$ and number of clinic appointments (>2) (AOR: 5.27, 95\% CI: 1.44-19.24, p-value: 0.012).

Conclusion: In conclusion, practices of proper medication management among stroke patients appeared poor. This could lead to increased risk of medication errors and mismanagement among stroke survivors. Female gender, higher socioeconomic status and higher number of clinic appointments could lead to better home MMP. Concerted health education should be provided especially in relation to medication management to improve home MMP in stroke patients in the community.

\section{Oral abstract 15}

The prevalence of gestational diabetes, associated risk factors and feto-maternal outcome among antenatal women attending health clinics in Terengganu

Rozimah $\mathbf{A L}^{1}$, Nurul Azreen $\mathrm{Y}^{2}$, Ranimah $\mathrm{Y}^{3}$, Zahrni $\mathrm{M}^{4}$, Tengku Bahiah TL ${ }^{5}$, Kamilah $\mathrm{M}^{6}$, Darisah L7 , Rohaiza $\mathrm{AK}^{8}$, Maira $\mathrm{H}^{9}$, Wan Ruzilasalwa WS ${ }^{4}$, Siti Aminah AM ${ }^{10}$, Mohd Sharil Iman $\mathrm{MH}^{11}$

${ }^{1}$ Family Medicine Specialist, Klinik Kesihatan Merchang, Marang, Terengganu; ${ }^{2}$ Faculty of Medicine, Universiti Sultan Zainal Abidin, Kuala Terengganu, Terengganu; ${ }^{3}$ Klinik Kesihatan Rahmat, Setiu, Terengganu; ${ }^{4}$ Klinik Kesihatan Kuala Dungun, Dungun, Terengganu; ${ }^{5}$ Klinik Kesihatan Padang Luas, Besut, Terengganu; 'Klinik Kesihatan Batu Rakit, Kuala Nerus, Terengganu; ${ }^{7}$ Klinik Kesihatan Bukit Tunggal, Kuala Nerus, Terengganu; ${ }^{8}$ Klinik Kesihatan Seberang Takir, Kuala Nerus, Terengganu; ${ }^{9}$ Klinik Kesihatan Manir, Kuala Terengganu, Terengganu; ${ }^{10}$ Klinik Kesihatan Kuala Berang, Hulu Terengganu, Terengganu; ${ }^{11}$ Klinik Kesihatan Merchang, Marang, Terengganu

Intoduction: Overall prevalence of diabetes mellitus (DM) 
among adult female in Malaysia was $18.3 \%$. One of the risk factors of DM is Gestational Diabetes Mellitus (GDM). The prevalence of GDM and feto-maternal outcome in Terengganu were never been reported before. This study examined the prevalence of GDM, its associated risk factors and to describe the feto-maternal outcome of women with GDM in Terengganu.

Methods: A cross-sectional study using 270 antenatal records of pregnant women who delivered in 2018 from 40 health care clinics in Terengganu. Sample size was calculated using Opinepi software and stratified random sampling was applied. Antenatal record book of all the selected cases were retrieved from each corresponding Health Clinic for sociodemographic data, risk factors for GDM and the feto-maternal outcome. The data was analysed using SPSS version 20.0.

Results: From 270 data reviewed, 264 (97.8\%) had been completed and included in the analysis. $98.9 \%$ were Malay and the rest $1.1 \%$ were Chinese. Majority of them were less than 35 years old, had secondary education level and had antenatal booking done in first trimester. The prevalence of GDM was $27.3 \%(n=72) .95 .8 \%(n=69)$ of the GDM women had good baseline glycemic control with HbA1c less/equal to $6.0 \%$. $23.1 \%$ on insulin while almost all seen by dietitian. $55.6 \%$ had BMI $>27 \mathrm{~kg} / \mathrm{m} 2$ at booking and $50 \%$ had first degree family history of DM. The prevalence of GDM women having history of GDM was higher than those in non-GDM (26.4\% vs 5.2\%). In logistic regression analysis, significant associated factors were family history of DM and personal history of GDM. The prevalence of caesarean section was higher among GDM women compared to non-GDM women (55.6\% vs $28.1 \%$ ). Only 62 of GDM women $(86.1 \%)$ underwent postnatal MOGTT in which $17.7 \%$ were found to have IFG and/ or IGT. For fetal outcome, neonatal jaundice, neonatal hypoglycaemia and preterm delivery was higher among infant of GDM mothers Conclusion: The prevalence of GDM among antenatal women attending primary health care clinics in Terengganu was significant. Significant association were seen for the family history of DM and history of GDM. Women with GDM had higher percentage adverse feto-maternal outcomes which can be reduced by proper pre-pregnancy care and optimisation of glucose control.

\section{Oral Abstract 16}

Experiences of Family Medicine Specialist (FMS) in providing healthcare for lesbian, gay, bisexual and transgender (LGBT) patients: A qualitative study

Siti Noorfadhilah $\mathbf{M A}^{1}$, Rosediani $\mathrm{M}^{1}$, Samsul $\mathrm{D}^{2}$, Maryam $\mathrm{MZ}^{1}$

${ }^{1}$ Department of Family Medicine, School of Medical Sciences, Universiti Sains Malaysia; ${ }^{2}$ Department of Family Medicine, Faculty of Medicine, International Islamic University Malaysia

Introduction: Depression is a common mental disorder associated with substantial disability. Moving towards community-oriented management of mental health problems, primary care doctors play a vital role in screening, identifying, and managing common mental health problems. This study aimed to examine the primary care doctor's attitude towards depression and determine its association with their sociodemographic factors, training background, and clinical experience.

Methods: A cross-sectional study is done between March to April 2018 amongst 144 government primary health care medical officers working in health clinics in Terengganu. The Revised Depression Attitude Questionnaire (R-DAQ), a validated 22-items Likert scale (0-5), was used to determine their attitude towards depression and its treatment in primary care. Respondents' sociodemographic factors and training experiences are collected using a self-administered questionnaire. The data was analyzed using the IBM SPSS statistics software version 19.0.

Results: The mean age of participants was 31 years old; majority were female (78.5\%) and Malay (94.4\%). The respondents demonstrate a good perspective about depression occurrence, recognition, and management (mean = 3.2). There is a significant lack of professional confidence in managing depression $($ mean $=1.4)$ and moderate pessimistic views on depression treatment outcomes (mean $=3.5)$. Doctors with a longer duration of clinical experience have more optimistic views about depression $(\mathrm{p}=0.013)$. In contrast, those with a personal history of depression and more frequent encounters with depressed patients have more pessimistic views $(\mathrm{p}=0.003, \mathrm{p}=0.017)$.

Conclusion: The study findings indicate the dire need for targeted training for primary care providers in managing common mental health problems.

\section{Oral Abstract 17 \\ Utility and Usability of the EMPOWER-SUSTAIN Desktop and Mobile Apps` among Primary Care Physicians and Patients with Metabolic Syndrome in Primary Care}

Maryam HD ${ }^{1,2}$, Anis Safura $\mathrm{R}^{1,2}$, Suraya $\mathrm{AR}^{1,2,3}$, Fakhrul Hazman Y, Mohamed Syarif MY², Noorhida B ${ }^{2}$, Siti Fatimah $\mathrm{BS}^{2}$, Mohamad Rodi I5 , Azlina Wati ${ }^{6}$, Nursuriati $\mathrm{J}^{4}$, Hapizah $\mathrm{N}^{1}$

${ }^{1}$ Institute of Pathology, Laboratory and Forensic Medicine (I-PPerForM), Universiti Teknologi MARA (UiTM); ${ }^{2}$ Department of Primary Care Medicine, Faculty of Medicine, UiTM; ${ }^{3}$ Cardio Vascular and Lungs Research Institute (CaVaLRI), Hospital UiTM; ${ }^{4}$ Department of Computer Science, Faculty of Computer \& Mathematical Sciences, UiTM; ${ }^{5}$ Department of Population Health \& Preventive Medicine, Faculty of Medicine, UiTM; ' Department of Psychiatry, Faculty of Medicine, UiTM

Introduction: The EMPOWER-SUSTAIN e-Health Intervention is a potential solution to improve self-management behaviour among patients with Metabolic Syndrome (MetS) in primary care. This study aimed to explore the utility and usability of newly developed EMPOWER-SUSTAIN Desktop and Mobile Apps $@$ among primary care physicians (PCP) and patients with MetS. 
Methods: This study was conducted at a university primary care clinic in Selangor using 'Think Aloud' qualitative method and Cognitive Task Analysis. Topic guide for the semi-structured interviews was developed based on the 10 Nielsen Heuristic. Utility testing was conducted among PCP. They were requested to 'Think Aloud' while they performed tasks assessing the desktop and mobile apps. Patients with MetS were given the mobile app to use 3 weeks before the usability testing. They were requested to 'Think Aloud' while performing tasks assessing mobile app. The interviews were audio and video recorded and transcribed verbatim. Data was managed using Nvivo software (version 12) for thematic content analysis.

Results: A total of 7 PCP and 9 patients were recruited. Six themes (efficiency of use, user control \& freedom, appearance $\&$ aesthetic features, clinical content, error prevention, and help \& documentation) emerged from the utility and usability testing. The PCP found the desktop app userfriendly. However, they had difficulty going to previous page, were unable to edit medication, found the interface immature and the colour too feminine. The PCP found the mobile app attractive and relevant sections were easy to find. However, they suggested bigger fonts for some parts and adding 'zoom' and 'swipe' functions. Patients found the mobile app user-friendly, with nice and simple interface, and straightforward language. It helped them understand their health better. However, they had difficulty understanding graphs due to absence of legends and could not edit/delete data in the My Self-Management section. Based on these feedbacks, both apps were refined to improve their utility and usability.

Conclusion: Overall, PCP and patients found the apps user-friendly with attractive interface. The apps were refined to improve efficiency of use, user control \& freedom, appearance \& aesthetic features, clinical content, and error prevention. The refined apps are ready for feasibility testing.

\section{Oral Abstract 18}

\section{Level of acceptance of executive diploma programme among Medical Officers: A prestudy survey}

\section{Anu Suria G1, Yafizah $Y^{1}$, Fathima Begum SMำ , Mohd Radzniwan AR ${ }^{1}$, Sharifah Najwa $S^{1}$, Muhammad Tauffik $M^{1}$ ${ }^{1}$ Faculty of Medicine and Health Sciences, Universiti Sains Islam Malaysia}

Introduction: The demand of postgraduate study in general practice in Malaysia is high. Currently, only permanent doctors are permitted to be enrolled into local master's programme in Malaysia. Therefore, the study aims to evaluate the level of interest of doctors to enrol in an Executive Diploma Program in General Practice. Its findings will be useful as a guide to develop an Executive Diploma for General Practice in our institution.

Methods: This study will be a cross-sectional study, commencing from January 2022 for 3 months. Sample size required for this study will be 476 where inclusion criteria will be contract and permanent medical officers, including foreign doctors practising in Malaysia. Self-administered questionnaire consisting of socio-demographic profile, reason to upgrade knowledge and level of acceptance towards Executive Diploma programme will be distributed via online platform such as google form. Relevant ethical approval will be obtained and data will be analysed using SPSS Software 25. Results: The socio-demographic profile, reason to upgrade knowledge and level of acceptance towards executive diploma programme will be analysed. The data on the level of acceptance toward a new executive diploma programme will be demonstrated as either high or low.

Conclusion: The future of medical officers in upgrading their carrier pathway is crucial. Hence developing postgraduate pathway option for them is important. Moreover, this assists in the quality of patient care, particularly at the primary care level. It is hoped this study finding could assist in developing such program effectively and efficiently.

\section{POSTER ABSTRACT}

\section{Poster Abstract 1 \\ U.N.G.G.A.S : Knowledge on HIV/AIDS Among Adolescents in Kuala Lumpur \& Putrajaya}

\author{
Nazrie $\mathbf{S}^{\mathbf{1}}$, Nurashma J ${ }^{2} \&$ Lilis Suriani $\mathrm{R}^{1}$ \\ ${ }^{1}$ Health Department of Kuala Lumpur \& Putrajaya, Malaysia; \\ ${ }^{2}$ Institutes for Health Behavioural Research, Ministry of Health \\ Malaysia
}

Introduction: AIDS poses a serious threat to young people, both in Malaysia and throughout the world.

Methods: A cross sectional school-based study was conducted to assess the level of knowledge towards HIV/ AIDS and to identify differences between gender and student groups with knowledge on HIV/AIDS. The study has been conducted few localities in Kuala Lumpur and Putrajaya, Malaysia using self-administrated UNGGAS tool.

Results: A total of 444 respondents recruited using simple random sampling and class registration list as sampling frame. Results shows the mean age of respondents was 15.46 years $(\mathrm{SD}=0.641)$ and mean score of knowledge towards HIV/AIDS was 4.144 (SD=1.240). Level of knowledge were found to be high among 261 respondents (58.8\%), moderate among 125 respondents $(28.2 \%)$ and the remain of 58 respondents $(13.1 \%)$ was categorized as low. An independent-samples t-test indicates there was significant difference in knowledge between male $(\mathrm{M}=3.922$; $\mathrm{SD}=1.388)$ and female $(\mathrm{M}=4.361 ; \mathrm{SD}=1.032), \mathrm{t}(442)=3.776, \mathrm{p}=$ .000 . Meanwhile, result of ANOVA shows there is difference between student groups and knowledge on HIV/AIDS $(p<.005)$. Student from PKD Lembah Pantai group has the highest mean score $(M=4.890 ; S D=.0424 ; n=100)$ followed by students from PKD Cheras group ( $M=4.680 ; \mathrm{SD}=.695$; $\mathrm{n}=100)$ and student from PKD Putrajaya group ( $\mathrm{M}=4.522$; $\mathrm{SD}=1.110 ; \mathrm{n}=44)$. Student from PKD Titiwangsa group has the lowest mean score for knowledge $(\mathrm{M}=3.240 ; \mathrm{SD}=1.471$, $\mathrm{n}=100$ ). It also shows that the $\mathrm{p}$-value is .000 and this is less than the alpha value of .05 . 
Conclusions: In conclusion, there is a need to highlight the knowledge on HIV/AIDS through health education programme especially among adolescent group. There is necessity to tailor suitable awareness school-based campaigns in disseminating information and education on HIV/AIDS as it can act as starting point for young people for a better understanding.

\section{Poster Abstract 2 \\ Long Term Benefit of a Targeted Diabetes Education Program: The KK Chini Experience}

Nor Azam K $\mathbf{K}^{1}$, Bustamanizan $\mathrm{B}^{2}$, Asyikin $\mathrm{Z}^{2}$, Hafizah $\mathrm{P}^{3}$ ${ }^{1}$ Department of Family Medicine IIUM, 25200 Kuantan, Pahang; ${ }^{2}$ Klinik Kesihatan Chini, 26690 Pekan, Pahang; ${ }^{3}$ Department of Community Medicine IIUM, 25200 Kuantan, Pahang

Introduction: An effective diabetes education may empower the patient in reinforcing self-care and a healthy lifestyle. We aim to evaluate the one-year outcome of an education program on a targeted group of diabetic patients at a primary health clinic.

Methods: This is a cohort survey recruiting poorly controlled diabetic patients into a locally designed education program at KK Chini, Pahang. The four-hour program consists of empowering the small group of 5-6 patients with self-care practices given by doctor, diabetes educator, nutritionist, occupational therapist, and pharmacist. The cohort of 37 patients participated in 2017 and 2018 with complete record were included in this study. All relevant diabetes management information before and one year after the program were obtained and analysed using SPSS 24.0 version.

Results: All participants were Malays and residents of FELDA's settlement. Men (68.6\%) and elderly aged > 60 $(65.7 \%)$ formed the majority. The mean age of participants was $60+7$. Before the program, mean HbA1c level was $9.8 \%$ and a significant reduction in mean HbAlc of $1.2 \%$ was shown one year after the program. For the same duration of time, there were no new incidence of co-morbidities (hypertension, dyslipidemia, overweight) or diabetic complications (CVD, eye, foot, CKD), and no significant changes in fasting blood glucose, serum creatinine and body weight was found among these patients. In term of treatment, the total dose/day of diabetic medications (ODAs, insulin) received at pre, and one-year post-program also showed no significant difference.

Conclusion: Diabetic education program which is structured, multi-disciplinary and targeted on poorly controlled patients was shown to be beneficial in a one-year outcome without the need for intensification of therapy.

\section{Poster Abstract 3}

Association Between Screen Time and Sleep Among Children Between 3 to 6 Years Old

Foo $\mathrm{SX}^{1}$, Marina $\mathrm{AN}^{2}$, Aparna $\mathrm{M}^{3}$, Afaaf $\mathrm{E}^{4}$,

Amy Pakiam DJy ${ }^{5}$, Hew SY ${ }^{6}$, Verna Lee $\mathrm{KM}^{7}$, Fa'iza $\mathrm{A}^{8}$
${ }^{1}$ Klinik Britain, Kuala Lumpur; ${ }^{2}$ Klinik Kesihatan Kuala Lumpur, Kuala Lumpur; ${ }^{3}$ Klinik Kesihatan Tanglin, Kuala Lumpur; ${ }^{4}$ Klinik Primer PPUKM Cheras; ${ }^{5}$ Klinik Kesihatan Putrajaya Presint 11; 'Klinik Kesihatan Putrajaya Presint 18; 7 International Medical University, Kuala Lumpur 8International Islamic University Malaysia, Kuantan

Introduction: It is very common now for very young children to be exposed to electronic devices. They require sufficient sleep for brain development, learning and character building. Excessive screen time may reduce their total duration and quality of sleep.To determine the association between screen time and sleep problems among children aged 3-6, specifically bedtime resistance, sleep anxiety and daytime sleepiness.

Methods: We conducted a cross-sectional study on 252 parents with children aged 3-6, attending four government clinics in Kuala Lumpur and Putrajaya from July-December 2019. Parents reported their socio-demographic data, type of gadgets and child's screen-time. Sleep problems were assessed using the validated and pilot tested Children's Sleep Habits Questionnaire (CSHQ) in English and Malay. Spearman's correlation was used to assess association of mean screen time with sleep duration, total CSHQ score and the specific subtypes of sleep problems.

Results: The commonest used gadget was television (97.8\%) and mobile phones (73.2\%). Mean daily screen time is $5.4 \pm 3.7$ hours and mean total sleep duration is $9.9 \pm 1.64$ hours. The mean age of first exposure to electronic devices is 28 months, mean total CSHQ score 55 (range 44-76) and mean sub-score for bedtime resistance, sleep anxiety and daytime sleepiness are 13.2, 8.0 and 12.8, respectively. Bivariate analysis revealed weak relationship between total screen time and sleep duration $(\mathrm{R}=-0.08, \mathrm{p}=0.23)$, CSHQ ( $\mathrm{R}=0.078, \mathrm{p}=0.24)$, bedtime resistance $(\mathrm{R}=0.056, \mathrm{p}=0.398)$, sleep anxiety $(R=0.003, p=0.963)$ and daytime sleepiness $(R=$ $-0.149, \mathrm{p}=0.02)$.

Conclusion: Despite high total CSHQ scores, our study showed no significant association between screen time and sleep problems among these children.

\section{Poster Abstract 4}

Prevalence of Depressiom Among Type 2 Diabetes Mellitus Patients in Selected Primary Care Clinics in Perak and It's Associated Factors

Charanjit Kaur KS ${ }^{1}$, Melinder Kaur D ${ }^{2}$, Najmi $\mathbf{A}^{3}$, Nora Syazwani $\mathrm{ME}^{4}$, Ong LK $\mathrm{LK}^{5}$, Parimala $\mathrm{S}^{6}$, Wong $\mathrm{PF}^{7}$ ${ }^{1}$ Klinik Kesihatan Jelapang, Perak; ${ }^{2}$ Klinik Kesihatan Gunung Rapat, Perak; ${ }^{3}$ Klinik Kesihatan pasir Pinji, Perak; ${ }^{4}$ Klinik Kesihatan Tanjung Malim, Perak; ${ }^{5}$ Klinik Ksihatan Setiawan, Perak; ${ }^{6}$ Klinik Menara Setia Triangle, Penang 7 Klinik Kesihatan Cheras Baru, KL

Introduction: Diabetes mellitus (DM) is becoming increasingly prevalent worldwide and is often associated with depression due to multiple factors. The primary objective of this study was to determine the prevalence of depression 
amongst Type 2 DM patients and specifically to determine the association between depression and socio-demographic and clinical factors.

Methods: This was a cross sectional study involving a total of 460 DM respondents. Subjects were screened for depression using the Patient Health Questionnaire (PHQ-9). Another questionnaire was also given to identify the factors linked to depression. A score of less than 4 was deemed not depressed and score of more than or equal to 4 was accepted as depressed.

Results: The prevalence of depression was 21.3\%. Patients with monthly income of RM2001- RM3000 were about 4 times (95\% CI: 0.12-0.60; $\mathrm{p}=0.001$ ) less likely to be depressed compared to monthly income of less than RM500. DM patients with cerebrovascular accident were 5 times more likely to be depressed $(\mathrm{OR}=5.15 ; 95 \% \mathrm{CI}$ : 1.88 , 14.06; $\mathrm{p}=0.001)$. Patients prescribed with prandial insulin were twice more likely to be depressed $(\mathrm{OR}=2.33$; 95\% CI: $1.13,4.82 ; \mathrm{p}=0.023)$ and premix insulin had a 3 -fold risk of being depressed $(\mathrm{OR}=3.04 ; 95 \% \mathrm{CI}: 1.54,6.01 ; \mathrm{p}=0.001)$. On the other hand, those prescribed with ACEi /ARB (OR=0.54; 95\% CI: 0.32, 0.91; $\mathrm{p}=0.021)$, Calcium Channel Blocker (OR=0.52; 95\% CI: 0.32, 0.84; $\mathrm{p}=0.007)$ or Antiplatelet (OR=0.56;95\% CI: 0.32, 0.97; $\mathrm{p}=0.038)$ were approximately twice less likely to be depressed, a significant protective factor of depression.

Conclusion: This study validates the strong relationship between diabetes and depression and its associated risk factors, hence screening for depression in primary care should be made mandatory.

\section{Poster Abstract 5}

\section{Danger Signs in Unwell Children Under Five: Can The Caregivers Identify Them?}

\section{Nik Fahmi MJ ${ }^{1}$, Nor Azam K ${ }^{1}$, Hafizah $\mathrm{P}^{2}$ \\ ${ }^{1}$ Department of Family Medicine, IIUM Kuantan Pahang; \\ ${ }^{2}$ Department of Community Medicine, IIUM Kuantan Pahang}

Introduction: Under the Sustainable Development Goals (SDG), one of its targets is to end all preventable deaths of children under five years old by 2030. In Malaysia, nearly $30 \%$ of the mortality cases are preventable and family factor including delay in seeking care is a known contributing factor. Hence, early detection of danger signs by caregivers is a key strategy. We aim to explore the caregivers' ability to identify danger signs of unwell children.

Methods: 393 caregivers of under five children who attended eight maternal and child health clinics in Kuantan, Pahang between January and August 2020 were recruited in this cross-sectional study. They were asked to identify the 10 danger signs from a checklist of 20 signs and symptoms for unwell children.

Results: Most caregivers were female (90.6\%) of Malay ethnicity $(89.3 \%)$ with a mean age of $32 \pm 6$. The mean age for their children was $24 \pm 18$ months. Out of the 10 danger signs, nine were correctly identified by most caregivers $(61.8 \%-81.9 \%)$, except for "reduce urine output" (33.8\%).
For the non-danger signs, the majority of caregivers identified "high fever" (80.9\%) and "persistent cough" (57\%) as danger signs while 5.1 to $39.9 \%$ of the caregivers chose the rest. Conclusion: Many of the caregivers in this study were able to correctly identify most of the danger signs in unwell children under five. This shows that empowering caregivers with home care and seeking early treatment is of paramount importance. On the same note, non-physical sign such as "reduce urine output" may need to be emphasized during safety netting advice.

\section{Poster Abstract 6}

\section{Effectiveness of Healthy Lifestyle Education in Diabetic Control in a Primary Care Clinic}

Tay CL, Nur Musfirah A, Yong KC, Mohd Azwan MN, Hafiszatulsima Z,, Ng WW, Mior Nurshafiq MMJ Simpang Health Clinic, Health District of Larut, Matang and Selama, Taiping, Perak, Ministry of Health Malaysia

Introduction: Four out of five diabetic Malaysians were unable to achieve good glycaemic targets. Insufficient health literacy among patients with diabetes is associated with worse glycaemic control. Thus this research is aimed to determine the impact of the Healthy Lifestyle Education (HLE) course to the diabetic control.

Methods: This is a comparative cross-sectional study. Medical records of patients with diabetes who either attended or notattended HLE course at Simpang Health Clinic from April 2018 to September 2018 were reviewed and study data extracted. Wilcoxon signed-ranks test, Pearson chi-square test, Fisher's exact test and Binary logistic regression were employed. Results: A total of 220 medical records including 106 HLE attendees and 114 non-attendees were recruited. Mean ages of attendees and non-attendees were 57.5 \pm 11.9 years and $60.8 \pm 9.9$ years respectively. The attendees achieved reduction in glycosylated haemoglobin (HbA1c), fasting blood sugar (FBS) and total cholesterol after HLE course $(\mathrm{p}<0.05)$. Those non-attendees had significant weight lost but increment in systolic blood pressure, HbA1c, LDL and insulin's total daily dose $(\mathrm{p}<0.05)$. Reduction in Fasting Blood Sugar (FBS) among the HLE Attendees was independently associated with HbA1c reduction $[\mathrm{p}=0.015$, odds ratio $(95 \% \mathrm{CI})=3.83(1.30-11.27)]$. Conclusion: Our work suggests that HLE has improved glycaemic and lipid control among patients with diabetes. Hence it should be executed at the primary care settings.

\section{Poster Abstract 7}

Foot Care Knowledge and Self-Care Practices Among Diabetics in Penang: A Primary Case Study

\author{
Ong $\mathrm{JJ}^{1}$, Azmil $\mathrm{SS}^{1}$, Kang $\mathrm{CS}^{2}$, Lim $\mathrm{SF}^{1}$, Mawardi $\mathrm{M}^{3}$, \\ Ooi GC ${ }^{4}$, Patel $\mathrm{A}^{1}$ \\ ${ }^{1}$ Penang State Health Department, Ministry of Health Malaysia; \\ ${ }^{2}$ Klinik Singapore, Georgetown, Pulau Pinang; ${ }^{3}$ Department \\ of Family Medicine, Faculty of Medicine \& Health Sciences, \\ Universiti Putra Malaysia, Selangor; ${ }^{4}$ Klinik Lim, Nibong \\ Tebal, Pulau Pinang
}


Introduction: In Malaysia, the prevalence of diabetes mellitus has been increasing annually and currently affects $18.3 \%$ of the population. Diabetic foot ulcer, a common complication of diabetes, is associated with high morbidity and mortality, consequently increasing health care expenditure. A previous study showed that foot care knowledge and foot self-care practices help to reduce development of ulcers. This study aims to identify the level of foot care knowledge and self-care practices among diabetic patients in the primary care setting. This study was to determine the level of foot care knowledge and foot self-care practices among diabetic patients in the primary care setting in Penang Island and it's determinants, and the correlation between level of foot care knowledge and self-care practices among diabetic patients.

Methods: A cross sectional study was performed on 311 diabetic patients who were registered to two government health clinics in Penang. Information regarding respondents' demographic status, foot care knowledge and foot selfcare practices were gathered using a self-administered questionnaire. Data were analysed using the SPSS 22. MannWhitney $U$ test and Kruskal Wallis test were applied in the analysis. Multiple linear regression was performed to identify the determinants. Correlation between knowledge and selfcare practice was determined using the linear regression model.

Results: The median age of respondents was 61 years old. Mostly were females (56.6\%), Malays (41.2\%) and unemployed (48.6\%). Median HbA1c level was 7.5\% and $42.8 \%$ of respondents had diabetes for 5 to 10 years. Lowest scores for knowledge and self-care practices were observed in foot skin care questions. Formal foot care education was found to be a significant predictor of foot care knowledge $(\mathrm{p}<0.05,95 \%$ CI $-1.102,-0.098)$. Foot care knowledge was significantly and positively correlated with foot self-care practices ( $\mathrm{p}<0.001,95 \%$ CI $0.548,0.727$ ).

Conclusion: Foot care knowledge has significant positive correlation with foot self-care practices. Empowering diabetic patients with foot care knowledge may lead to significantly better foot self-care practices.

\section{Poster Abstract 8}

Measuring Implementation Fidelity of The Family Doctor Concept (FDC) Program in Public Primary Care Clinics in Selangor, Malaysia

Reffien MAM ${ }^{1}$, Aniza I ${ }^{1 *}$, Sulong $S^{1}$, Tengku PZTB ${ }^{2}$, Noridah $\mathrm{MS}^{2}$, Nazrila $\mathrm{HN}^{2}$

${ }^{1}$ Department of Community Health, Faculty of Medicine, National University of Malaysia; ${ }^{2}$ Family Health Development Division, Ministry of Health Malaysia

Introduction: The FDC program was introduced as a part of the national health care reform agenda in late 2013. The study aims to measure implementation fidelity of the program using a checklist.

Methods: The study was conducted from December 2019 to January 2020 involving six clinics in Selangor. The checklist used was developed by a panel of experts, and was found to be valid and reliable. It contains 15 items, which are divided into 4 components (population registration, formation of primary care team, integrated client treatment, and monitoring and evaluation). Assessment was done by a scoring system based on information obtained from interviews, observations and documents.

Results: The median total score was $7.00 \pm 1.75$, with a maximum of 9 and a minimum of 5 . KKD was found to have the highest total score (9), followed by KKE (7), KKC (7), KKA (7), KKB (6) and KKF (5). All of the clinics were scored 1 out of 5 for population registration, and monitoring and evaluation components. For formation of primary care team component, most of the clinics were scored 2 out of 4, except for KKD that was given full score. For integrated client treatment components, the majority of the clinics were scored 3 out of 4 , except for KKB and KKF that were scored 2 and 1 , respectively.

Conclusion: In conclusion, the majority of the clinics were found to have low implementation fidelity, especially for two of its component. Further study is required to investigate how and why it happened.

\section{Poster Abstract 9}

Non-compliance and its associated factors to iron chelation therapy among transfusion dependent thalassaemia patients in Kelantan and Terengganu.

Allen Shiun CC ${ }^{1}$, Nani D ${ }^{1}$ Siti Suhaila bt $\mathrm{MY}^{1}$, Nurul Fatihah $\mathrm{A}^{5}$, Maryam $\mathrm{MZ}^{1}$, Najib $\mathrm{MY}^{3}$, Norsarwany $\mathrm{M}^{2}$, Rosline $\mathrm{H}^{4}$, Wan Zaidah $\mathrm{A}^{4}$, Alwi Z1 ${ }^{5,6}$

${ }^{1}$ Department of Family Medicine, School of Medical Sciences, Universiti Sains Malaysia, 16150 Kubang Kerian, Kelantan, Malaysia; ${ }^{2}$ Department of Pediatrics, School of Medical Sciences, Universiti Sains Malaysia, 16150 Kubang Kerian, Kelantan, Malaysia; ${ }^{3}$ Unit of Biostatistics and Research Methodology, School of Medical Sciences, Universiti Sains Malaysia, 16150 Kubang Kerian, Kelantan, Malaysia; ${ }^{4}$ Department of Hematology, School of Medical Sciences, Universiti Sains Malaysia, 16150 Kubang Kerian, Kelantan, Malaysia; ${ }^{5}$ Malaysian Node of the Human Variome Project, School of Medical Sciences, Universiti Sains Malaysia, 16150 Kubang Kerian, Kelantan, Malaysia; ${ }^{6}$ Human Genome Centre, School of Medical Sciences, Universiti Sains Malaysia, 16150 Kubang Kerian, Kelantan, Malaysia.

Introduction: Patients with transfusion dependent thalassaemia (TDT) require lifelong blood transfusion unless receiving haemopoietic stem cell transplant. Iron overload is a common complication of it, and effective iron chelation therapy (ICT) improves survival. Despite the availability of variable of iron chelating agents, there is limited data on compliance with them. This study aims to determine the proportion of non-compliance to ICT and its associated factors among TDT patients in Malaysia.

Methods: A cross-sectional study was conducted among TDT patients age 9 years old and above who were on ICT, attending three tertiary hospitals in Kelantan and 
Terengganu. The patient's socio-demographic, clinical and chelators history were obtained. The patient's compliance to chelators was assessed using Malay Version Medication Compliance Questionnaire (MCQ). Social support was assessed by using the revised Malay version Medical Outcome Study Social Support Survey (MOS). Associated factors with non-compliance were analysed using simple and multiple logistic regression, while the association between MRI result and serum ferritin with compliance status was explored by using either Chi-square or Fisher's exact test.

Results: The proportion of non-compliance to ICT was $24.7 \%$. Age, restriction of activity and latest serum ferritin level of $>1000 \mu \mathrm{g} / \mathrm{L}$ were significantly associated with noncompliance. Significant association was observed between non-compliance and MRI liver, but not with MRI cardiac result.

Conclusion: The non-compliance to ICT among TDT patients in Kelantan and Terengganu is $24.7 \%$. Providing motivational interview, enhanced patient's self-empowerment and psychological support are recommended to improve the non-compliance aspect among patients on ICT.

\section{Poster Abstract 10}

\section{Audit on Dyslipidemia management of Type2 Diabetes Mellitus patients in Changkat Jering Heatlh clinic, Perak.}

\section{Lai MK ${ }^{1}$, Leow $\mathrm{SN}^{1}$, Noor Afiqah $W^{1}$, Nor Ashikin $\mathrm{MR}^{1}$ ${ }^{1}$ Klinik Kesihatan Changkat Jering}

Introduction: Type 2 Diabetes mellitus (DM2) is commonly associated with dyslipidemia. This increased the risk of cardiovascular disease if not treated to target. This audit was conducted to review dyslipidemia management among DM2 patients in Changkat Jering health clinic.

Methods: By using convenience sampling, a total of 251 DM2 patients with active followup were selected from January 2021 to April 2021. Their medical record book with demographic, investigation and medication data were collected and analyzed with SPSS 23.

Results: The mean age of patients was 61.3 (11.3) years old, median HbA1c was 7.5\% (2.5), LDL-C was $2.7 \mathrm{mmol} / \mathrm{L}$ (1.2), and triglyceride (TG) was $1.3 \mathrm{mmol} / \mathrm{L}(0.9), 53 \%$ (133) were obese and 96\% (241) were associated with comorbid. $94.8 \%$ (238) were unable to achieve the target LDL-C, and $31.5 \%$ (79) were unable to achieve target TG. $88.1 \%$ (221) of patients was on simvastatin, and $8.8 \%(22)$ was on atorvastatin. Further analysis showed $28.7 \%$ (72) patients were prescribed with simvastatin $40 \mathrm{mg}$ ON with concomitant amlodipine $10 \mathrm{mg}$ OD, out of which, $97.2 \%$ (70) were unable to achieve target LDL-C.

Conclusion: This audit demonstrates that majority patients in Changkat Jering health clinic was not treated to target LDL. There is a need to consider early switch to high intensity statins (e.g., atorvastatin) for all eligible high-risk patients to reduce patients' morbidity and mortality outcome.
Poster Abstract 11

Factors Associated with High Obstructive Sleep Apnea Risk among Adult Patients Attending UITM Primary Care Clinic

\begin{abstract}
Sharifah Azimah WA ${ }^{1}$, Suraya $\mathrm{AR}^{2,3,4}$, Anis Safura $\mathrm{R}^{2,3}$, Mariam $\mathrm{M}^{5}$

${ }^{1}$ Klinik Kesihatan Kapit, Jalan Bleteh, 96800, Kapit, Sarawak; ${ }^{2}$ Department of Primary Care Medicine, Faculty of Medicine, Universiti Teknologi MARA, Selayang Campus, 68100 Batu Caves, Selangor; ${ }^{3}$ Institute of Pathology, Laboratory and Forensic Medicine (I-PPerFORM), Universiti Teknologi MARA, Sungai Buloh Campus, Jalan Hospital, 47000, Sungai Buloh, Selangor; ${ }^{4}$ Cardio Vascular and Lungs Research Institute (CaVaLRI), Hospital Universiti Teknologi MARA (HUiTM), Jalan Hospital, Sungai Buloh, Selangor; ${ }^{5}$ Department of Public Health Medicine, Faculty of Medicine, UiTM, Sg Buloh Campus, Jalan Hospital, 47000, Sungai Buloh, Selangor
\end{abstract}

Introduction: Obstructive sleep apnea (OSA) increases risk of cardiovascular diseases (CVD), yet underdiagnosed. OSA and metabolic syndrome (MetS) synergistically increase CVD morbidity and mortality. Data is scarce on prevalence and factors associated with high OSA risk including MetS. Hence, this study aimed to determine the prevalence and factors associated with high OSA risk.

Methods: A cross-sectional study was conducted at UiTM Primary Care Clinics between June 2018-August 2018. Adults aged $\geq 18$ years old who fulfilled the inclusion and exclusion criteria were recruited using systematic random sampling. A validated Malay version of Berlin Questionnaire (BQ-M) was used. MetS was defined by the Joint Interim Statement (JIS) criteria. Socio-demographic, clinical parameters, fasting lipid profile and fasting blood glucose data were obtained. Multiple logistic regression was used to determine factors associated with high OSA risk.

Results: 408 participated with a response rate of $93.8 \%$. Mean age was $56.04 \pm 13.00$ years, with more males ( $\mathrm{n}=$ 209, 51.2\%), Malays $(\mathrm{n}=346,84.8 \%)$ and non-smokers $(n=283,69.4 \%)$. The overall prevalence of high OSA risk was 39.7\% (95\% CI: 34.94\%,44.47\%). There were high proportion of MetS in the overall study population $(60 \%, n$ $=245)$ and among high OSA risk group $(75.3 \%, \mathrm{n}=122)$. Being male [OR 2.16,(95\% CI:1.41,3.31)], having visceral obesity [OR 2.07, (95\% CI:1.12,3.84)] and clinically diagnosed with MetS [OR 2.06, (95\%CI: 1.24, 3.43)] were more likely to have high OSA risk.

Conclusion: Patients attending sub-urban primary care clinics are at risk of having high OSA risk. Thus, it is imperative to screen for OSA particularly among high risk groups attending the primary care clinics especially male, individuals with visceral obesity and MetS. 
Poster Abstract 12

Stress, anxiety and depression among antenatal women in Terengganu: The prevalence and associated factors.

Nurul Azreen $Y^{1}$, Nurul Izza $Y^{1}$, Nur Aiza I' ${ }^{1}$, Zaleha J'2, Siti Aminah $\mathrm{AM}^{3}$, Mazlinah $\mathrm{M}^{4}$, Nor Kasmawati J $\mathrm{J}^{5}$, Azlin $\mathrm{A}^{6}$, Juhaida ${ }^{6}$, Salmiah $S^{7}$, Azila ${ }^{8}$

${ }^{1}$ Faculty of Medicine, Universiti Sultan Zainal Abidin, Terengganu; ${ }^{2}$ Klinik Kesihatan Maran; ${ }^{3}$ Klinik Kesihatan Kuala Berang; ${ }^{4}$ Klinik Kesihatan Bukit Payong, Kuala Berang; ${ }^{5}$ Klinik Kesihatan Permaisuri, Bandar Permaisuri; ${ }^{6}$ Klinik Kesihatan Hiliran; ${ }^{7}$ Klinik Kesihatan Ajil, ${ }^{8}$ Klinik Kesihatan Pulai Chondong, Kelantan

Introduction: The study aimed to determine prevalence of depression, anxiety and stress and its associated factors among antenatal patients in primary care health clinics in Terengganu.

Methods: This cohort study was conducted among 688 antenatal women who attended 22 health clinics in Terengganu. They were screened for mental health problems using Depression, Anxiety, Stress Score (DASS) questionnaire and followed up at the first, second and third trimester from August 2019 to May 2020. A detailed structured questionnaire with items on sociodemographic information, background medical and obstetric problems, psychosocial factors was used. Other variables include any negative events happened throughout pregnancy and perceived support from spouse as well as their family. Logistic regression analyses between the dependent and independent variables were conducted using IBM SPSS ver. 20.0.

Results: The prevalence of stress, anxiety and depression among antenatal patients in Terengganu was $21.4 \%$ as determined by abnormal DASS. All of the mental health problems highly occurred during the first trimester whereby $69.9 \%$ of antenatal women experienced depression, $63.5 \%$ had anxiety while $52.1 \%$ had stress. They mostly had mild depression, anxiety and stress throughout all trimesters. Lower educational level, unplanned pregnancy, past obstetric history, and having a medical problem during pregnancy were significantly associated with having mental health problems in pregnancy.

Conclusion: Mental health problems frequently occurred in all trimesters of pregnancy. Knowing this indicates its screening and early detection in pregnancy are important to allow early interventions to prevent future mental disorders and improve feto-maternal outcome.

\section{Poster Abstract 13}

Epidemiological trends of NCD in Beladin, Pusa, Sarawak: A cross sectional study

Stephen JM${ }^{1}$, Syafiq AIZ2 ${ }^{2}$ Johnny $\mathrm{P}^{3}$

${ }^{1}$ Klinik kesihatan Beladin, Sarawak; ${ }^{2}$ Pejabat kesihatan daerah Saratok, Sarawak; 'Pegawai kesihatan bahagian Betong, Sarawak

Introduction: Non communicable diseases such as diabetes mellitus and hypertension is a burden to the country's health economy. As such, the detection rate of new diabetes and hypertension in our country is still below par. This is due to the lack of awareness of the community voluntarily come for health screening until symptoms set in. This study was done to create a new approach of new case detection rate of diabetes and hypertension in a small community in Beladin, Betong

Methods: The community were screened using glucometer and blood pressure machines in their home from 16 Mac to 20 October 2019. 75\% ( $\mathrm{n}=3296)$ were unemployed with 9\% $(\mathrm{n}=388)$ self employed and 6\% $(\mathrm{n}=261)$ are fishermen and labourer $(\mathrm{n}=279)$ respectively. 34\% $(\mathrm{n}=1503)$ are primary school educated, while $32 \%(n=1403)$ are secondary school educated and 31\% (1358) are illiterate.

Results: A total of $40.5 \%(n=4382)$ Beladin population were eligible, all being Malay with 2117 male and 2269 female screened. Preexisting 7\% $(n=288)$ of diabetic and $14 \%$ $(\mathrm{n}=608)$ hypertensive patients were detected. Newly diagnosed diabetic and hypertensive patients had an increment of $2 \%$ $(\mathrm{n}=213)$ and $6 \%(\mathrm{n}=115)$ respectively.

Conclusion: This study shows significant undiagnosed chronic disease among the community. This new approach ensures the communities are screened for NCD diseases earlier prior to symptom occurrence with early intervention of healthcare workers at ground level.

\section{Poster Abstract 14}

The prevalence of depression and its associated factors among married female diabetic patients in Kuantan, Pahang

Md Aris MA ${ }^{1}$, KF Tan ${ }^{1}$, Mohd Rus $\mathrm{R}^{2}$

${ }^{1}$ Department of Family Medicine, Kulliyyah of Medicine, International Islamic University Malaysia, Jalan Sultan Haji Ahmad Shah, 25200 Kuantan Pahang; '2Department of Community Medicine, Kulliyyah of Medicine, International Islamic University Malaysia, Jalan Sultan Haji Ahmad Shah, 25200 Kuantan Pahang

Introduction: Depression is reported to affect more women than men in Malaysia. The objective of this study was to determine the prevalence of depression and its associated factors among married female diabetic patients attending primary care clinics.

Methods: A cross-sectional study was conducted from August to December 2020 in 5 primary care clinics in Kuantan. Data were collected using an interviewer-administered questionnaire. Level of depression was assessed with the 9-item patient health questionnaire (PHQ-9). The Malay version of female sexual function index (MVFSFI) was used to measure sexual dysfunction. Chi square and Man-Whitney U test were used to determine the association between all independent variables with depression.

Results: There were 241 respondents. The mean age of respondents were 52.9 (SD=7.75). A majority were Malay (79.3\%), Muslim (80.5\%), housewives (68\%), and menopaused (54.4\%). The prevalence of depression was $6.2 \%$ (PHQ score $>10)$. Severe depression was present in 1 subject, while moderately severe depression was present in 4 subjects. 
There were 10 subjects who experienced moderate depression, and 48 experienced mild depression. The study revealed that medication use and duration of diabetes were significantly associated with depression ( $\mathrm{p}=0.03)$. Female sexual dysfunction was significantly associated with depression $(\mathrm{p}=0.028)$.

Conclusion: The prevalence of depression among married female diabetic patients was $6.2 \%$. The clinician should take the initiative to screen for depression among female diabetic patients who had prolonged duration of diabetes or those who were on insulin. Besides that, the clinician may screen for female sexual dysfunction if the patients presented with depressive symptoms.

\section{Poster Abstract 15}

Fetal Heart Coin: Cardiac Rhabdomyoma not to be missed

Md Aris MA1, Khamis@ Roslee NS ${ }^{2}, \mathbf{K F}$ Tan $^{1}$

${ }^{1}$ Department of Family Medicine, Kulliyyah of Medicine,

International Islamic University Malaysia, Jalan Sultan Haji

Ahmad Shah, 25200 Kuantan Pahang; ${ }^{2}$ Balok Health Clinic,

Kuantan, Pahang, Malaysia

Introduction: Fetal cardiac rhabdomyoma is a rare disease and most of the cases are detected incidentally during an antenatal ultrasound.

Case Report: We describe a case discovered during antenatal ultrasonography at 29 weeks of gestation during routine antenatal follow-up in primary care clinic. Multiple hyperechogenic round shape masses were seen in the fetal cardiac. The patient was subsequently referred to a tertiary hospital for a further detailed scan and fetal echocardiography. The diagnosis of fetal cardiac rhabdomyoma was confirmed prenatally. After birth, the baby was followed up with a pediatric cardiologist with regular echocardiography. Management was mainly conservative at the time being as there was no sign of obstruction.

Conclusion: This case emphasises the necessity of ultrasound during a prenatal check-up in recognising abnormal findings. The primary care physician plays a critical role in detecting unusual findings and referring patients to a tertiary hospital for further shared care and management.

\section{Poster Abstract 16}

Patient Profile and Drug Use in A Special Upper Respiratory Tract Infection Clinic Based in A Primary Healthcare Setting During Covid-19 Pandemic in Malaysia: A Cross Sectional Study

Ooi $Z^{1}$, Nurul Abidah $\mathrm{MG}^{2}$, Nang Juniza $\mathrm{NZ}^{3}$, Chan $\mathrm{HK}^{4}$ Norsiah $\mathrm{MN}^{3}$, Noor Liani $\mathrm{H}^{3}$,Mohd Firdaus $\mathrm{AB}^{3}$, Mohd Redhuan $\mathrm{AM}^{3}$

${ }^{1}$ Klinik Kesihatan Batu Pahat, Jalan Kluang, Taman Limpoon, 83000 Batu Pahat, Johor; ${ }^{2}$ Hospital Sultanah Bahiyah, Km 6, Jln Langgar, Bandar Alor Setar, 05460 Alor Setar, Kedah; ${ }^{3}$ Klinik Kesihatan Bandar Alor Setar, Aras 1 Blok A, Darul Aman Highway, 05100 Alor Setar, Kedah; ${ }^{4}$ Clinical Research Centre, Hospital Sultanah Bahiyah, Km 6, Jln Langgar, Bandar Alor Setar, 05460 Alor Setar, Kedah
Introduction: Upper respiratory tract infection (URTI) is a common encounter at the primary care level. Its management is particularly challenging due to its similar symptoms to COVID-19 infection. Our study evaluated the profile and antibiotic use in patients seeking care from a special community-based URTI clinic during the COVD-19 pandemic.

Methods: This was a cross-sectional study. Data were obtained from the medical records of patients visiting the URTI clinic located at the Alor Setar Primary Healthcare Center between March and April 2020.

Results: Of the $587(3.16 \%)$ patients received treatment, most were male (60.6\%) and aged between 20 and 39 years $(35.5 \%)$. Their most common symptoms were cough $(68.4 \%)$, fever $(31.6 \%)$, runny nose $(24.6 \%)$ and sore throat $(24.1 \%)$. Most of them were diagnosed with acute nasopharyngitis $(52.5 \%)$, followed by acute pharyngitis $(18.6 \%)$ and acute tonsillitis (5.3\%). The antibiotics constituted $6.2 \%$ of the medications used. Only 26 out of 35 patients diagnosed with URTI received antibiotics, yielding an antibiotic use rate of only $4.45 \%$ for URTI relative to the overall drug use. Acute tonsillitis was found to be more common in children aged $<12$ years $(\mathrm{p}<0.001)$, while the presence of cough and runny nose were more commonly indicative of acute nasopharyngitis $(p<0.001)$. Sore throat was more likely to be the symptom of acute pharyngitis $(\mathrm{p}<0.001)$ and acute tonsillitis $(\mathrm{p}<0.001)$.

Conclusion: Despite the challenges during the COVID-19 pandemic, the finding suggests that the patients with URTIlike symptoms were properly managed, and the antibiotic use remained reasonable.

\section{Poster Abstract 17}

Functional Disabilities and Its Associated Factors among Elderly Residents in Private Nursing Homes in Kuantan

\section{Mohd Aznan MA ${ }^{1}$, Mazilah $\mathbf{M}^{1}$, Razman $\mathrm{MRs}^{2}$, Suhaiza $\mathrm{S}^{1}$ \\ ${ }^{1}$ Department of Family Medicine, Kulliyyah of Medicine, International Islamic University Malaysia, Jalan Sultan Haji Ahmad Shah, 25200 Kuantan Pahang; ${ }^{2}$ Department of Community Medicine, Kulliyyah of Medicine, International Islamic University Malaysia, Jalan Sultan Haji Ahmad Shah, 25200 Kuantan Pahang}

Introduction: Residing in nursing home poses a greater challenge for elderly physically and mentally. The study aimed to determine the prevalence of functional disabilities and its associated factors among elderly in private nursing homes in Kuantan.

Methods: A cross-sectional study conducted at thirteen private nursing homes in Kuantan, Pahang. A total of 105 elderly residents aged 60 years and above were recruited to complete the interview-based questionnaire. Modified Barthel Index (MBI), Malay Geriatric Depression Scale (M-GDS-14), and Elderly Cognitive Assessment Questionnaire (ECAQ) were used to measure activities of daily living (ADL), depressive symptoms and cognitive impairment respectively. Descriptive statistics was used to measure the prevalence and logistic regression to explore the 
association of functional disabilities with the background variables.

Results: The mean age of the respondents was 74.36 (SD 9.09) with majority being male (51.4\%), Chinese (66.7\%), single/divorced $(73.4 \%)$, received formal education $(77.1 \%)$ and had chronic disease (84.8\%). $45.7 \%$ of the residents are cognitively impaired, and $39.1 \%$ have depressive symptoms. The overall prevalence of functional disabilities was $41.9 \%$, with the most common functional dependence were climbing stairs (93.3\%). There were significant associations between functional disabilities with cognitive impairment AOOR 2.658, CI 95\% (1.109-6.371) \} and depressive symptoms \{AOR 3.27, CI 95\% (1.349-7.926) \}.

Conclusion: Functional disability among the residents place greater physical and financial demands on providers. This study revealed nearly half of the elderly residing in nursing homes are ADL dependent. Residents with cognitive impairment and depressive symptoms should receive greater attention to prevent further disability and dysfunction.

\section{Poster Abstract 18}

\section{Exploring how patient engagement is initiated in diabetes} care

Syahnaz $\mathbf{M H}^{1}$, Idayu $\mathrm{BI}^{2}$, Nasrin $\mathrm{J}^{3}$, Rafidah $\mathrm{B}^{4}$, Shalisah $\mathrm{S}^{5}$ ${ }^{1}$ Department of Family Medicine, Universiti Kebangsaan Malaysia Medical Centre, Kuala Lumpur, Malaysia; ${ }^{2}$ Department of Community Health, Universiti Kebangsaan Malaysia Medical Centre, Kuala Lumpur, Malaysia; ${ }^{3}$ Freelance Public Health Physician, Dhaka, Bangladesh; ${ }^{4}$ Department of Psychiatry, University of Cyberjaya, Cyberjaya, Selangor, Malaysia; ${ }^{5}$ Department of Psychiatry, Universiti Kebangsaan Malaysia Medical Centre, Kuala Lumpur, Malaysia

Introduction: Patient engagement has been recognised as a crucial factor in managing a chronic illness like diabetes. Past studies had reported good clinical outcomes among patients who actively participated in their care. However, currently, there is still a lack of understanding on how local patients could engage with diabetes care. Therefore, this study aimed to understand better how patients begin to engage in diabetes self-management.

Methods: A qualitative study was conducted from August till December 2018 in UKM primary care clinic. Ten participants with diabetes were purposively sampled and indepth interviews were done. The data were analysed with thematic analysis.

Results: The analysis generated three main themes. "Learning what to do" is the first theme, constituting the process of learning from family members, peers and healthcare professionals that helped to define their role in diabetes self-management. In the beginning, patients desired a more gradual approach, i.e. "Taking one step at a time." They also wished to start with a more straightforward task. When patients had a low perceived ability to perform certain selfmanagement tasks, such as lifestyle changes, they engaged with family members or peers to help: "Engaging with someone for challenging tasks".
Conclusion: Knowledge of self-management and self-efficacy are the most fundamental aspects of patient engagement in diabetes care. Healthcare providers should ensure a comprehensive diabetes education is being provided for their patients and suited their needs. Having a health partner or buddy among family members or peers would assist patients in developing their confidence to adopt self-management behaviour.

\section{Poster Abstract 19}

\section{A Retrospective Audit on Cloxacillin Prescription in Primary Health Care Facilities in Kuala Selangor}

Farhani $S^{1}$, Nurul Nadia $\mathbf{B}^{2}$, Nor Anizah $\mathrm{M}^{3}$, Sharifah Nurul Aida $\mathrm{SG}^{4}$, Sharmilee $\mathrm{R}^{5}$, Nuraini $\mathrm{D}^{6}$, Noor Rafizah $\mathrm{AA}^{7}$ ${ }^{1}$ Klinik Kesihatan Tanjong Karang, Jalan Sungai Kajang, 45000 Kuala Selangor, Selangor; ${ }^{2}$ Klinik Kesihatan Bukit Cherakah, Jalan Rizab Masjid, Kampung Bukit Cherakah, 45800 Jeram, Selangor; ${ }^{3}$ Klinik Kesihatan Kuala Selangor, Jalan Klinik, Bandar Malawati, 45000 Kuala Selangor, Selangor; ${ }^{4}$ Klinik Kesihatan Bestari Jaya, 45600 Batang Berjuntai, Selangor; ${ }^{5}$ Klinik Kesihatan Ijok, JKR 1087, Jln 14, Ijok, 45700 Batang Berjuntai, Selangor; ${ }^{6}$ Klinik Kesihatan Jeram, Jalan Klang 45800 Jeram Kuala Selangor; ${ }^{7}$ Pejabat Kesihatan Daerah Kuala Selangor, Jalan Semarak, 45000 Kuala Selangor, Selangor

Introduction: The Defined Daily Dose (DDD) usage of Cloxacillin in PKD Kuala Selangor ranged between 3.0 to 8.7 in which it is higher than the WHO recommendation. It is the second most prescribed antibiotic for skin and soft tissue infection in Malaysia. However, resistant patterns for Cloxacillin are alarming. This study focuses on appropriate usage of Cloxacillin to prevent further antimicrobial resistance.

Methods: This is a retrospective cross-sectional audit, conducted in seven public health clinics in the Kuala Selangor district from January 2020 to March 2020. All Cloxacillin prescriptions within this duration were selected. The audit format was adapted from the antimicrobial stewardship protocol (AMS). Appropriate Cloxacillin use is based on the accuracy of diagnosis, indication of antibiotic, correct usage based on the National Antibiotic Guideline (NAG), pharmacological name, dose, frequency and duration.

Results: A total of 259 prescriptions were collected. The majority were for aged $\geq 40$ years old, $48.6 \%(n=126)$ and male, 63.7\% ( $n=165)$. Cloxacillin was prescribed mostly for skin and subcutaneous tissue infection 64.1\% ( $n=166)$. Diagnosis was reported as accurate in $63.7 \%(n=165)$. Those with accurate diagnosis, had the correct indication $79.4 \%$ $(131 / 165)$; correct usage $51.5 \%(85 / 165)$; correct name 69.7\% (115/165); correct frequency 55.2\% (91/165); correct dose 55.8\% (92/165), and correct duration 47.3\% (78/165). Appropriate prescription was reported in 41.8\% (69/165). Conclusion: There is still room for improvement for the appropriate usage of Cloxacillin in the outpatient clinic setting. Measures to improve appropriate Cloxacillin use and adherence to the National Antibiotic Guideline is crucial. 
Poster Abstract 20

\section{Prevalence of severely elevated low-density lipoprotein cholesterol (LDL-c) in public primary care clinics in Klang Valley, Malaysia}

Aisyah $\mathbf{K}^{1}$, Anis Safura $\mathrm{R}^{1,2, \$}$, Arina $\mathrm{B}^{1}$, Johanes $\mathrm{DK}^{1}$, Suraya $\mathrm{AR}^{1,2,4}$, Siti Fatimah $\mathrm{BS}^{2}$, Noorhida $\mathrm{B}^{2}$, Mohamed Syarif $\mathrm{MY}^{2}$, Hasidah $\mathrm{AH}^{2,3}$, Hapizah $\mathrm{MNi}^{1}$, Nadeem $\mathrm{Q}^{3}$ ${ }^{1}$ Institute of Pathology, Laboratory and Forensic Medicine (I-PPerForM), Universiti Teknologi MARA, Sungai Buloh Campus, Jalan Hospital, 47000 Sungai Buloh, Selangor, Malaysia; 'Department of Primary Care Medicine, Faculty of Medicine, Universiti Teknologi MARA, Selayang Campus, 68100 Batu Caves, Selangor, Malaysia; ${ }^{3}$ Division of Primary Care, School of Medicine, Faculty of Medicine \& Health Sciences, University of Nottingham, United Kingdom; ${ }^{4}$ Cardio Vascular and Lungs Research Institute (CaVaLRI), Hospital Universiti Teknologi MARA (HUiTM), Jalan Hospital, 47000 Sungai Buloh, Selangor, Malaysia

Introduction: Lifelong exposure to elevated low-density lipoprotein cholesterol (LDL-c) has been shown to cause atherosclerotic cardiovascular disease (ASCVD). Consumption of high saturated fat diet has been associated with elevated LDL-c. Apart from this, severely elevated LDL-c (defined as $\geq 4 \mathrm{mmol} / \mathrm{L}$ ) could also be a clinical feature of Familial Hypercholesterolaemia (FH), an autosomal dominant genetic condition. Therefore, the objective of this study was to determine the prevalence of severely elevated LDL-c among adults attending the public primary care clinics in Klang Valley, Malaysia.

Methods: This was a cross-sectional study conducted in 5 public primary care clinics in Selangor and Wilayah Persekutuan Kuala Lumpur from September 2020 to May 2021. The sampling frame was adult patients aged $\geq 18$ years old with LDL-c results recorded in the electronic medical record (EMR). Sociodemographic data and LDL-c readings from 2018 to 2020 were extracted from the EMR. Severely elevated LDL-c levels were classified into 4 categories: 4.0 $4.9 \mathrm{mmol} / \mathrm{L} ; 5.0-5.9 \mathrm{mmol} / \mathrm{L} ; 6.0-6.9 \mathrm{mmol} / \mathrm{L}$ and $\geq 7$ $\mathrm{mmol} / \mathrm{L}$.

Results: Out of 74,431 patients' records screened from the EMR, 25,038 (33.6\%) patients had severely elevated LDL-c of $\geq 4 \mathrm{mmol} / \mathrm{L}$. The mean age was $55.5(\mathrm{SD} \pm 5.4)$ years. Out of the 25,038 patients $69.2 \%$ had LDL-c of $4.0-4.9$ $\mathrm{mmol} / \mathrm{L}, 23.6 \%$ had LDL-c of $5.0-5.9 \mathrm{mmol} / \mathrm{L}, 5.6 \%$ had LDL-c of $6.0-6.9 \mathrm{mmol} / \mathrm{L}$ and $1.6 \% \mathrm{had}$ LDL-c of $\geq 7$ $\mathrm{mmol} / \mathrm{L}$. More than half of the patients with severely elevated LDL-c in each of these categories were women i.e. 59.6\%, $58.9 \%, 56.6 \%$ and $55.9 \%$, respectively.

Conclusion: Severely elevated LDL-c is highly prevalent in these primary care clinics. Consumption of high saturated fat diet could be the cause for this. However, these patients should be screened further for other underlying causes of dyslipidaemia, including FH. Early diagnosis and treatment, including aggressive lifestyle modification are paramount to prevent premature ASCVD in these patients.
Poster Abstract 21

Clinical detection of Familial Hypercholesterolaemia (FH) using Familial Hypercholesterolaemia Case Ascertainment Tool (FAMCAT), Simon Broome (SB) Criteria and Dutch Lipid Clinic Criteria (DLCC) in public primary care clinics in Klang Valley, Malaysia

\begin{abstract}
Johanes D $\mathbf{k}^{\mathbf{1}}$, Anis Safura $\mathrm{R}^{1,2,}$, Aisyah $\mathrm{K}^{1}$, Mohamed Syarif $\mathrm{My}^{2}$, Noorhida $\mathrm{B}^{2}$, Suraya $\mathrm{Ar}^{1,2,4}$, Siti Fatimah $\mathrm{BS}^{2}$, Hasidah $\mathrm{AH}^{2,3}$, Hapizah $\mathrm{MN}^{1}$, Nadeem $\mathrm{Q}^{3}$

${ }^{1}$ Institute of Pathology, Laboratory and Forensic Medicine (I-PPerForM), Universiti Teknologi MARA, Sungai Buloh Campus, Jalan Hospital, 47000 Sungai Buloh, Selangor, Malaysia; 'Department of Primary Care Medicine, Faculty of Medicine, Universiti Teknologi MARA, Selayang Campus, 68100 Batu Caves, Selangor, Malaysia; ${ }^{3}$ Division of Primary Care, School of Medicine, Faculty of Medicine \& Health Sciences, University of Nottingham, United Kingdom; ${ }^{4}$ Cardio Vascular and Lungs Research Institute (CaVaLRI), Hospital Universiti Teknologi MARA (HUiTM), Jalan Hospital, 47000 Sungai Buloh, Selangor, Malaysia
\end{abstract}

Introduction: Familial Hypercholesterolaemia (FH) is a common autosomal dominant genetic condition characterised by severely elevated low-density lipoprotein cholesterol (LDL-c). However, FH is often underdiagnosed and undertreated in primary care. Several clinical diagnostic criteria are available to detect FH. Therefore, the objective of this study was to clinically detect FH using FAMCAT, Simon Broome (SB) Criteria and Dutch Lipid Clinic Criteria (DLCC) among adults attending the public primary care clinics in Klang Valley, Malaysia.

Methods: This was a cross-sectional study conducted in four public primary care clinics in Selangor and Wilayah Persekutuan Kuala Lumpur from September 2020 to May 2021. The sampling frame was adult patients aged $\geq 18$ years old with LDL-c results recorded in the electronic medical record (EMR). Patients with LDL-c levels of $\geq 4.0 \mathrm{mmol} / \mathrm{L}$ were invited to participate and sent a flyer about the study via social media messaging service. Those who agreed to participate were scheduled for an appointment to be seen at the primary care clinic, where their eligibility were assessed according to the inclusion and exclusion criteria. Written informed consent was obtained from those who were eligible and agreed to participate. Data for socio-demographics, FAMCAT, SB and DLCC c variables were collected.

Results: A total of 1565 patients with LDL-c of $\geq 4.0$ $\mathrm{mmol} / \mathrm{L}$ were invited to participate via social media messaging service, of which 205 patients agreed to participate and were given an appointment to be seen at the primary care clindiagnostiic. However, 196 (12.5\%) patients turned up for their appointment and were found to be eligible and recruited for the study. Out of 196, 123 (62.8\%) fulfilled the FH clinical diagnostic criteria by FAMCAT and/or SB and/ or DLCC. Out of 123, 39 (31.7\%) fulfilled all three clinical diagnostic criteria.

Conclusion: A high proportion of patients with LDL-c of $\geq 4.0 \mathrm{mmol} / \mathrm{L}$ fulfilled the $\mathrm{FH}$ clinical diagnostic criteria. 
These patients need to be assessed further by genetic testing, looking for mutations in the $\mathrm{FH}$ candidate genes such as LDLR, APOB and PCSK9. Early diagnosis and treatment of $\mathrm{FH}$ are vital to prevent premature atherosclerotic cardiovascular diseases.

\section{Poster Abstract 22}

Prenatal diagnosis and management of fetal hydronephrosis in primary care: a case report

Nadia Hamimah $\mathrm{K}^{1}$, Siti Shafiatun $\mathrm{M}^{2}$

${ }^{1}$ Klinik Kesihatan Putatan, Jln Pasir Putih 88200 Putatan, Sabah; ${ }^{2}$ Klinik Kesihatan Cheras

Introduction: Fetal hydronephrosis (FH) is among the most common congenital anomaly identified during prenatal ultrasound. Although $\mathrm{FH}$ is usually physiological, it can be associated with various urinary tract abnormalities. We report a case of $\mathrm{FH}$ and highlight the importance of its detection and management during prenatal period in primary care.

Case report: $\mathrm{FH}$ was discovered during prenatal ultrasound of a 27-year old pregnant lady at 34 weeks of gestation at our health clinic. It was subsequently confirmed post delivery as bilateral hydronephrosis and hydroureter with grade 5 vesicoureteric reflux and left partial duplex system which the latter is a rare aetiology of FH. Complicated with poor social background, shared care with Maternal-Fetal Medicine (MFM) specialist during prenatal period was delayed. However, through effective communication with tertiary care, the child managed to receive appropriate care post delivery.

Conclusion: Prenatal diagnosis of $\mathrm{FH}$ and prompt management of its condition during the early neonatal period are important to minimise adverse outcomes of the affected child after delivery.

\section{Poster Abstract 23}

Effective contraceptive practise and its associated factors among women with uncontrolled diabetes in Terengganu.

Hassan BM${ }^{1}$, Nik Nor Ronaidi $\mathrm{NM}^{2}$, Aniza $\mathrm{AA}^{2}$, Shaiful Ehsan $S^{3}$, Anisah $J^{4}$, Kasemani $E^{5}$, Sunita $S^{5}$ ${ }^{1}$ Family Medicine Unit, Faculty of Medicine, Universiti Sultan Zainal Abidin, Medical Campus, Jalan Sultan Mahmud, 20400, Kuala Terengganu, Terengganu, Malaysia; ${ }^{2}$ Community Medicine Unit, Faculty of Medicine, Universiti Sultan Zainal Abidin, Medical Campus, Jalan Sultan Mahmud, 20400, Kuala Terengganu, Terengganu, Malaysia; ${ }^{3}$ Department of Family Medicine, Kulliyyah of Medicine, UIAM Kuantan Campus, Jalan Sultan Ahmad Shah, 25200 Kuantan, Pahang. Malaysia; ${ }^{4}$ Obstetrics and Gynaecology Unit, Faculty of Medicine, Universiti Sultan Zainal Abidin, Medical Campus, Jalan Sultan Mahmud, 20400, Kuala Terengganu, Terengganu, Malaysia; ${ }^{5}$ Terengganu State Health Department, Ministry of Health Malaysia, 5th Floor, Wisma Persekutuan, Jalan Sultan Ismail, 20920 Kuala Terengganu, Terengganu, Malaysia

Introduction: Women with uncontrolled diabetes should practice effective contraception to avoid unplanned pregnancy. This study aimed to determine the prevalence of the effective contraceptive practice and its associated factors among women with uncontrolled diabetes.

Methods: A cross-sectional study was conducted among women of reproductive age with uncontrolled diabetes. Subjects were recruited among diabetic women attending multiple governments health clinics in Terengganu using a multistage random sampling method. Effective contraception was defined as hormonal contraception (oral, injection, implant), intrauterine contraceptive device, and bilateral tubal ligation. Data were collected using a self-administered questionnaire and medical record review. Multiple logistic regression was used to determine the associated factors for effective contraception practice.

Results: A total of 305 women with uncontrolled diabetes aged 19 to 49 participated in this study. The proportion of effective contraceptive practice was 36.4\% (95\% CI: 42.2, 53.5). The effective contraceptive practice was significantly associated with unemployment (Adj.OR: 2.05; 95\% CI:1.22, 3.44), poor knowledge on preconception care (Adj.OR: 0.6; 95\% CI:0.37, 0.99), and poor attitude on preconception care (Adj.OR: 0.44; 95\% CI; 0.23, 0.87).

Conclusion: The prevalence of effective contraceptive practice was unsatisfactory. Effective contraception practice should be emphasized on all women with uncontrolled diabetes. Preconception counselling focusing on glucose control and suitable contraception should be compulsory for this population to improve their knowledge and attitude towards preconception care

\section{Poster Abstract 24}

Does system-based intervention reduce incidence of Type 2 diabetes and improve the metabolic profiles postnatally in women with history of gestational diabetes? A prospective, randomized controlled study (SBI-GDM)

Tan $\mathbf{S F}^{1}$, Azahadi $\mathrm{O}^{2}$, Hazlin $\mathrm{M}^{3}$, Lee $\mathrm{YS}^{4}$, Mohd Khairi MN5 , Noor Azreen $\mathrm{M}^{6}$, Salmah $\mathrm{N}^{7}$, Nazrila Hairizan $\mathrm{N}^{8}$, Lee KW ${ }^{9}$, Ching SM ${ }^{9}$

${ }^{1}$ Klinik Kesihatan Pelabuhan Klang, Ministry of Health Malaysia; ${ }^{2}$ Institute of Public Health, Ministry of Health Malaysia ; ${ }^{3}$ Klinik Kesihatan Teluk Panglima Garang, Ministry of Health Malaysia; ${ }^{4}$ Klinik Kesihatan Selayang, Ministry of Health Malaysia; ${ }^{5}$ Klinik Kesihatan Shah Alam Seksyen, Ministry of Health Malaysia; ' Pejabat Kesihatan Daerah Klang, Ministry of Health Malaysia; ${ }^{7}$ Klinik Kesihatan Cheras Batu 9, Ministry of Health Malaysia; ${ }^{8}$ Family Health Development Division, Ministry of Health Malaysia; ${ }^{9}$ Department of Family Medicine, Faculty of Medicine and Health Sciences, Universiti Putra Malaysia

Introduction: In Malaysia, GDM affected $27.9 \%$ of pregnant ladies. SBI GDM is to study whether systembased interventions can reduce the incidence of T2DM and improve metabolic profiles in women with history of GDM. Methods: SBI-GDM was a prospective, randomized controlled study conducted in 17 primary care clinics in 
Selangor. Subjects were randomised into intervention and control groups. Individualized health education by trained staff nurse and medical officer were given to the intervention group in each follow up visit. Women in both arms were followed up for 24 months postnatally.

Results: A total number of 666 respondents were recruited in this study. Women with tertiary education in the control group were higher compared with the intervention group $(60.2 \%$ versus $39.8 \%, p=0.001)$. Overall incidence of diabetes was $18.4 \%$ with $20 \%(n=30)$ and $17 \%(n=31)$ in intervention and control groups respectively which was not statistically significant $(\mathrm{p}=0.487)$. LDL was significantly lower in the intervention group $3.3 \mathrm{mmol} / \mathrm{L}$ compared to the control group $3.9 \mathrm{mmol} / \mathrm{L}, \mathrm{p}=0.001$.

Conclusion: The incidence of T2DM in our study was higher compared to the finding from a systematic review of randomized controlled trials. Almost one fifth of mothers with history of GDM developed T2DM at 2 years postpartum. Our study shows there is no difference in terms of incidence of T2DM between intervention and control groups. Interventions with more intensive approaches are recommended to prevent or delay the development of T2DM.

\section{Poster Abstract 25}

Validity and Reliability of the Malay IOWA Infant Feeding Attitude Scale (IIFAS-M) among mothers with infants in Malaysia

\section{Nurjasmine $\mathrm{AJ}^{1}$, Hazwani $\mathbf{H H}^{\mathbf{1}, \mathbf{2}}$, Karimah Hanim $\mathrm{AA}^{3}$,}

Nur Azam K ${ }^{1}$

${ }^{1}$ Department of Family Medicine, Kulliyyah of Family Medicine, International Islamic University Malaysia; ${ }^{2}$ Department of Community and Family Medicine, Faculty of Medicine and Health Sciences, University Malaysia Sabah; ${ }^{3}$ Department of Community Medicine, Kulliyyah of Family Medicine, International Islamic University Malaysia.

Introduction: Maternal knowledge, attitude and practices are factors that can influence infant feeding choices, particularly breastfeeding. Valid and reliable instruments on maternal attitudes towards infant feeding choices in the Malay language are still insufficient for the Malaysian population.

Objectives: To translate and validate the Malay version of the IOWA Infant Feeding Attitude Scale (IIFAS).

Methods: Four independent language experts performed forward and backed translation of IIFAS. A cross-sectional study involving 272 mothers with infants below one-year-old was conducted between 8th March 2021 to 6th June 2021 through social media platforms using the final versions of the translated questionnaires. Construct validity, Cronbach's alpha coefficients and test-retest reliability were calculated. Descriptive analysis and factor analysis were performed.

Results: The online questionnaire received a total of 522 responses. Two hundred seventy-two responses fulfilled the inclusion criteria and were analysed. Most of the respondents were Malay women with the mean age of 30 years old and have two children with the youngest's age was 5-month-old. The validity and reliability measures of the Malay IIFAS were acceptable. Pearson correlation shows all the items to be significant at 0.01 . KMO is 0.657 , and Bartlett's test of sphericity is significant at 0.001. Principal component analysis extracted six factors with value ranges from 0.4 to 0.8. The Cronbach's alpha value for the scale was 0.62 and ranged from 0.2 to 0.6 . Test-retest showed good reliability $(\mathrm{r}=0.802, \mathrm{p}<0.01)$. The IIFAS Malay showed acceptable internal consistency reliability.

Conclusion: The Malay version of IIFAS is a valid, reliable, and locally accepted questionnaire to assess maternal attitude towards infant feeding in the Malaysian population. However, a locally developed questionnaire may be made in the future to produce a better statistical accepted questionnaire.

\section{Poster Abstract 26}

\section{2 in 1: COVID-19 Infection Reveals Tuberculosis}

Nur Izdiani ${ }^{1}$, Farah Diyana ${ }^{1}$, Siti Umi ${ }^{1}$, Rosnah ${ }^{1}$ ${ }^{1}$ Klinik Kesihatan Taman Ehsan

Introduction: During the COVID-19 pandemic, tuberculosis (TB) is still one of the leading infectious and most common respiratory diseases that cause high morbidity and mortality in Malaysia.

Case report: This case illustrates a co-incidental occurrence of respiratory infection of a lady who initially presented with asymptomatic COVID infection after having close contact at her workplace. Baseline chest $\mathrm{x}$-ray showed atelectasis of right upper zone. Sputum culture for M. tuberculosis in the hospital revealed Mycobacterium tuberculosis complex but no sensitivity test was done due to insufficient colony. She was discharged well and referred to the health clinic for further management. A full tuberculosis work-up was conducted despite her being asymptomatic. She was reported to have active tuberculosis and treatment for pulmonary tuberculosis was initiated. After 2 months of treatment, MTB C\&S revealed mono-drug resistant. She was then referred to a Respiratory physician. Upon further history, she was encircled to have contact with immediate family member who had drug resistant tuberculosis. In view of mono-drug resistant $\mathrm{TB}$, mass screening was done at her workplace including family members. IGRA test was done to 185 people with 13 persons detected as positive. She is now doing well with modified TB regime which consists of levofloxacin, rifampicin, ethambutol and pyrazinamide.

Conclusion: Considering the high worldwide prevalence of TB in Malaysia and the increasing burden of COVID-19, the co-infection seems more likely to be a co-incidental occurrence rather than a causal association which should not be missed in differential diagnosis of respiratory infection. 
Poster Abstract 27

\section{Internal Jugular Phlebectasia in Child; Now You See It Now You Don't}

Mohd Shaiful ES ${ }^{1}$, Muhammad Nasri $\mathrm{AB}^{2}$, Muhammad Zul Hazmi AZ ${ }^{1}$

${ }^{1}$ Department of Family Medicine, Kulliyyah of Medicine, International Islamic University Malaysia, Jalan Sultan Haji Ahmad Shah, 25200 Kuantan Pahang; ${ }^{2}$ Department of Otorhinolaryngology, Hospital Sultan Ismail Petra, Kuala Krai, Kelantan

Introduction: Neck swelling carries multiple differential diagnosis as various structures present within the neck region. Structural defects of the vascular wall such as phlebectasia mainly affect internal jugular vein causes swelling over lateral side of neck and represented by soft compressible swelling during increased intrathoracic pressure. Clinical suspicion could be made when observing the mass appears or increased in size upon straining, and diagnosis could be confirmed using noninvasive imaging modalities such as neck ultrasound with color Doppler. Although most phlebectasia cases rarely require intervention, prompt diagnosis will exclude other devastative causes of neck swelling that may require urgent medical attention and treatment.

Case report: We report a case of internal jugular vein phlebectasia in a 6-year-old girl that was initially noticed by her parents due to present of lateral neck swelling upon straining and shouting.

Conclusion: This case emphasises the necessity of decisive investigation and prompt diagnosis in any case presentation of neck swelling. The primary care practitioners play great role to assess patient's presentation holistically and channel the patient towards appropriate investigation to reach the correct diagnosis.

\section{Poster Abstract 28}

Prevalence And Factors Associated With Psychological Distress Among Patients With Hypertension In A Primary Care Clinic

\section{Wen $\mathrm{HL}^{1 *}$, SM Ching ${ }^{2}$ \\ ${ }^{1}$ Masters of Medicine (Family Medicine) candidate, Department of Family Medicine, Faculty of Medicine and Health Sciences, Universiti Putra Malaysia, 43400 Serdang, Malaysia; \\ ${ }^{2}$ Department of Family Medicine, Faculty of Medicine and Health Sciences, Universiti Putra Malaysia, Serdang, Selangor 43400, Malaysia}

Introduction: Literature shows psychological distress worsens hypertension's control. Relevant study is lacking in Malaysia. This study examines the prevalence and factors associated with depression, anxiety and stress among patients with hypertension in a primary care clinic.

Methods: A cross-sectional study was conducted using the 21item depression, anxiety stress scale (DASS-21) questionnaire to measure psychological distress
Results: A total of 389 patients were recruited with response rate of $99.5 \%$. Mean age of the study population was 60.1 years with $44.7 \%$ male respondents. Prevalence of psychological distress was $28.8 \%$, followed by anxiety (21.3\%), depression (16.2\%), and stress (13.9\%). According to multiple logistic regression, uncontrolled blood pressure $\quad(\mathrm{p}<0.001)$, worry about hypertension's complications $(\mathrm{p}<0.001)$, and physically inactive $(\mathrm{p}=0.001)$ are significantly associated with depression. Uncontrolled blood pressure $(\mathrm{p}<0.001)$, worry about hypertension's complications $(p<0.001)$, no formal education $(p=0.012)$, and working $(\mathrm{p}=0.004)$ significantly associated with anxiety. Lastly, uncontrolled blood pressure $(\mathrm{p}<0.001)$, worry about hypertension's complication $(\mathrm{p}<0.001)$, and low household income $(\mathrm{p}=0.010)$ significantly associated with stress.

Conclusion: Uncontrolled blood pressure control and worry about hypertension's complications are the most important independent risk factors. Clinicians should be more vigilant to identify high-risk patients for screening and further intervention.

\section{Poster Abstract 29}

The effect of structured physical activity during pregnancy on maternal health and fetal outcomes: A systematic review

Nurjasmine $\mathrm{AJ}^{1}$, Farah Hanani $\mathbf{M N}^{1}$, Karimah Hanim $\mathrm{AA}^{2}$ Mohammad $\mathrm{CM}^{1}$

${ }^{1}$ Department of Family Medicine, Kulliyyah of Medicine, International Islamic University Malaysia, Jalan Sultan Haji Ahmad Shah 25200 Kuantan, Pahang; ${ }^{2}$ Department of Community Medicine, Kulliyyah of Medicine, International Islamic University Malaysia, Jalan Sultan Haji Ahmad Shah 25200 Kuantan, Pahang

Introduction: Current guidelines recommend pregnant women to be physically active. It has been shown that exercise during pregnancy contributes to positive impacts on maternal and fetal outcomes. The aim of this review was to comprehensively assess and determine the effects of structured physical activity on maternal health and fetal outcome.

Methods: Literature was retrieved from electronic databases, namely PubMed, Google Scholar, Scopus. Medline, Cochrane Library and Science Direct published from 2015 to 2020. Clinical trials published in English involving lowrisk, uncomplicated pregnancies evaluating the effects of structured physical activity during pregnancy on maternal and fetal outcome were included. Outcome assessed were gestational weight gain, lumbopelvic pain, gestational diabetes mellitus, pre-eclampsia, premature delivery, and birth weight. The authors assessed the risk of bias in all eligible studies using Revman Software.

Results: Twenty studies were included in this systematic review. Structured physical activity during pregnancy did not cause an adverse effect on gestational duration and birth weight. The majority of the studies reported a positive or neutral effect on the incidence of gestational diabetes, 
pre-eclampsia, and gestational weight gain. Three studies demonstrated that exercise did help to reduce lumbopelvic pain during pregnancy.

Conclusion: Exercise during pregnancy is beneficial for maternal and fetal health outcomes when practiced according to recommendations. This systematic review is the first to suggest that structured physical activity is associated with significant benefits related to maternal and fetal outcomes. Therefore, it is recommended that healthcare providers to encourage and develop tailored exercise programs to improve maternal health and fetal outcome.

Keywords: structured physical activity, maternal and fetal outcomes, systemic review

\section{Poster Abstract 30}

\section{The effectiveness of UNICEF/WHO 20-hour} breastfeeding Course in Improving Health Professionals' Knowledge, Attitudes and Practice towards Breastfeeding in Malaysia: A quasi experimental study

Nurjasmine $\mathbf{A J}^{\mathbf{1}}$, Karimah $\mathrm{HAA}^{2}$, Hamizah $\mathrm{I}^{3}$

${ }^{1}$ Department of Family Medicine, Kulliyyah of Family Medicine, International Islamic University Malaysia Jalan Sultan Ahmad Shah, 25200 Kuantan Pahang; ${ }^{2}$ Department of Community Medicine, Kulliyyah of Family Medicine, International Islamic University Malaysia. Jalan Sultan Ahmad Shah, 25200 Kuantan Pahang; ${ }^{3}$ Department of Obstetrics and Gynaecology, Kulliyah of Medicine, International Islamic University, Jalan Sultan Ahmad Shah, 25200 Kuantan Pahang

Introduction: The UNICEF/WHO 20-hour Breastfeeding Course was designed as part of a global program to promote, support and protect breastfeeding. The module focuses on preparing healthcare workers on breastfeeding knowledge and supportive measures to help pregnant women and mothers to breastfeed their infant. Hence, we determined the effectiveness of this course on the level of knowledge, attitude and practice towards breastfeeding among nurses.

Methods: A quasi-experimental study was conducted at three-points (pre-intervention, post intervention and 3 months post intervention) on the level of knowledge, attitude and practice among two group of nurses working in Sultan Ahmad Shah Medical Centre@IIUM. One group $(n=90)$ received the intervention which was the 20-hour Breastfeeding Course which included information and training needed. The other group $(\mathrm{n}=90)$ received no intervention. The study was conducted from Dec 2019 till March 2020. A self-administered questionnaire was used to measure the knowledge, attitude and practice. The higher the score indicates higher level of knowledge, good attitude and practice. Results: The mean age was 27 years old for both groups, mean years of experience are 4 years and 2 years in the respective groups and majority of them had diploma. There is significant difference in the mean scores in knowledge, attitude and practice between the intervention and control group regardless of time (mean diff: 1.905, $\mathrm{p}<0.001 ; 4.227, \mathrm{p}<0.001 ; 3.51$, $\mathrm{p}<0.001)$ after controlling the years of experience.
Conclusion: The UNICEF/WHO 20-hour Breastfeeding Course was effective in increasing the level of knowledge, attitude and practice among nurses.

\section{Poster Abstract 31}

Incidence and FactorsS Associated With Diabetic Retinopathy Among Diabetic Patients in Klinik Kesihatan Cheras

Nadia Hamimah $\mathbf{K}^{1}$, Siti Shafiatun $\mathrm{M}^{2}$, Nur Faizah $\mathrm{H}^{3}$ ${ }^{1}$ Family Medicine Specialist, Ministry of Health Malaysia, Klinik Kesihatan Putatan; 'Consultant Family Medicine Specialist, Ministry of Health Malaysia, Klinik Kesihatan Cheras; ${ }^{3}$ Medical Officer, Ophthalmology Department, Hospital Melaka

Introduction: Diabetic retinopathy (DR) is the leading cause of vision loss in developing countries. We aimed to determine the incidence and factors associated with DR among diabetic patients in Klinik Kesihatan Cheras.

Methods: Diabetic records of patients who undergone fundoscopy between September 2018 to August 2019 were randomly selected and reviewed. Those with ungradable fundus photos or incomplete data were excluded. A total of 209 samples were analysed.

Results: The mean age of patients was 63.5 years and the majority were Chinese (56.5\%). The median duration of DM was 6.0 years and the mean HbAlc was $6.7 \%$. The majority of patients $(74.2 \%)$ were on oral therapy and had co-morbidities such as hypertension (86.6\%), dyslipidemia (96.2\%) and nephropathy (68\%). The incidence of DR was 9.6\% where 9 of them had maculopathy and 1 had Advanced Diabetic Eye Disease. The presence of microalbuminuria $(p=0.02)$, proteinuria $(p=0.03)$, and high triglyceride level $(\mathrm{p}=0.02)$ are significantly associated with DR. No significant association between duration of diabetes, blood pressure, and other laboratory profiles with DR.

Conclusion: DR is prevalent in diabetic patients. The presence of nephropathy and hypertriglyceridemia are significantly associated with DR. Regular screening and appropriate management of modifiable risk factors are vital to prevent vision-threatening retinopathy.

\section{Poster Abstract 32}

Prevalence of depression, anxiety, stress symptoms and the related factors among caregivers of children with Autism Spectrum Disorder in Kuantan, Pahang

Puteri Fatin NMAT ${ }^{1}$, Samsul D ${ }^{1}$, Mohammad CM ${ }^{1}$, Muhammad Zubir $\mathrm{Y}^{2}$, Rozanizam $\mathrm{Z}^{3}$, Noorul Amilin $\mathrm{H}^{3}$ ${ }^{1}$ Department of Family Medicine, Kulliyyah of Medicine, International Islamic University Malaysia, Jalan Sultan Ahmad Shah, 25200 Kuantan Pahang; ${ }^{2}$ Community Medicine, Kulliyyah of Medicine, International Islamic University Malaysia, Jalan Sultan Haji Ahmad Shah, 25200 Kuantan Pahang; ${ }^{3}$ Kulliyyah of Medicine, International Islamic University Malaysia, Jalan Sultan Haji Ahmad Shah, 25200 Kuantan Pahang. 
Introduction: Raising a child with autism spectrum disorder (ASD) can be challenging and causing psychological distress for most caregivers. The objective of the study is to determine the prevalence of depression, anxiety, stress symptoms and the related factors among caregivers of children with ASD in Kuantan, Pahang.

Methods: A cross-sectional study was conducted from December 2019 to December 2020. Caregivers of children with ASD attending National Autism Society of Malaysia (NASOM) Kuantan, psychiatry clinic and occupational therapy unit at Hospital Tengku Ampuan Afzan Kuantan and caregivers who joined PIANIS (Persatuan Ibubapa Anak Istimewa Pahang) were recruited using a convenience sampling method. Malay version of Depression Anxiety Stress Scales (DASS 21) and Brief COPE questionnaire were used. Statistical analyses used were independent t-test, chisquared test, and binary logistic regression.

Results: This study recruited 152 respondents. The majority were Malays (97.4\%) and women (60.5\%). 14.5\% had depressive symptoms, $16.4 \%$ had anxiety symptoms and $13.2 \%$ had stress symptoms. The most commonly used coping strategy was engagement (Mean=23.1, $\mathrm{SD}=6$ ). Disengagement was the only factor significantly related to depression symptoms ( $\mathrm{AOR}=1.49, \mathrm{CI}=1.16-1.91)$, anxiety symptoms $(\mathrm{AOR}=1.41, \mathrm{CI}=1.14-1.76)$ and stress symptoms $(\mathrm{AOR}=1.25$, CI 1.01-1.55).

Conclusion: Nearly one-sixth of the caregivers of children with ASD had symptoms of depression, anxiety and stress. Healthcare providers including primary care doctors should conduct more screening for depression, anxiety and stress symptoms among caregivers of children with ASD and provide psychoeducation on the appropriate coping strategies.

\section{Poster Abstract 33}

Prevalence of depression, anxiety and stress and its association with nicotine dependency among adult smokers attending primary care clinics.

Moideen $\mathrm{K}^{1}$, Masri $\mathrm{M}^{2}$, Low $\mathrm{CLY}^{3}$, Mansor $\mathrm{MD}^{4}$, Lim $\mathrm{CH}^{5}$, Chua $\mathrm{CL}^{6}$, Miskan $\mathrm{M}^{7}$, Ambigga $\mathrm{K}^{7}$

${ }^{1}$ KK Batu 2.5 Kemaman Terengganu; ${ }^{2}$ KK Kinabutan Tawau Sabah; ${ }^{3}$ KK Peringgit Melaka; ${ }^{4}$ KK Ayer Keroh Melaka; ${ }^{5}$ Klinik Melaka Cheng, Melaka; ${ }^{6}$ Klinik Bestari Cheng Melaka; ${ }^{7}$ Primary Care Medicine Unit, Faculty of Medicine and Defence Health, National Defence University of Malaysia, Malaysia

Introduction: Cigarette smoking is one of the predominant contributors to death and morbidity worldwide. The GATS (Global Adult Tobacco Survey) in 2011 divulged that the prevalence of current smokers in Malaysia was 23.1\%. To determine the prevalence of depression, anxiety and stress and its association with nicotine dependency among adult smokers attending primary care clinics in Malacca.

Methods: This was a cross-sectional study conducted at 4 primary care clinics in Melaka between 1st July 2019 to 31st August 2019 among current smokers aged above 18 years old. Socio-demographic Questionnaire, Depression Anxiety Stress Scale (DASS-21) and Fagerstrom Test of Nicotine
Dependence were utilized. Logistic regression tests were used to determine associations between depression, anxiety and stress with nicotine dependency.

Results: A total of 350 participants consented to the study with a response rate of $92 \%$. The age of respondents ranged from 18 years to 78 years and the mean age was $37.5 \pm 13.3$ years. The mean duration of smoking was 17.35 years. Prevalence of anxiety was $52.5 \%$, stress was $35.4 \%$ and depression was $26.4 \%$. There were statistically significant associations of nicotine dependency with depression $(\mathrm{p}<0.001$, CI $95 \%$ 0.031-0.100); anxiety $(\mathrm{p}<0.001$, CI $95 \%$ $0.033-0.101)$ and stress ( $\mathrm{p}<0.001$, CI 95\% 0.024-0.089).

Conclusion: Mental health screening is essential among smokers in primary care to identify psychological issues earlier for prompt referral and treatment.

\section{Poster Abstract 34}

\section{The prevalence and factors associated with mental health status among youths in Malaysia}

\section{Mohd Azlan ${ }^{1}$, Mohammad Asraffuddien B ${ }^{1}$, Fadzilah M2 ${ }^{1}$ Master of Science Student, Family Medicine Department, Faculty of Medicine and Health Sciences, Universiti Putra Malaysia; ${ }^{2}$ Medical Lecturer and Family Medicine Specialist, Family Medicine Department, Faculty of Medicine and Health Sciences, Universiti Putra Malaysia}

Introduction: The prevalence of mental health disorders has shown an increase in numbers especially among the youths. This has become another major health burden in the community. A severe mental illness may alter one's behaviour, emotion and cognition which could harm oneself and the community. It is important to study the factors that are associated with mental health status among the youngster as this can help both intervention and prevention programs. This study aimed to investigate the factors associated with mental health status among youth in Malaysia.

Methods: This was a pilot, survey-based, cross-sectional study on youths who currently attending higher institutions in Malaysia. A convenience sampling was used to collect participants and all data collection were done via an online platform. A detailed structured questionnaire with items on sociodemographic information and psychosocial factors was used. Depression, Anxiety, Stress Score (DASS-21) questionnaire was administered to assess the symptoms of depression, anxiety and stress among the youths. Data were analysed using SPSS 26.

Results: A total of 30 respondents were recruited. The prevalence of anxiety, depression and stress was 63.3\%, 40\% and $33.3 \%$ respectively. Results from binary logistic regression reported depression was only significantly associated with locality (AOR 95\% CI): 19.582 (1.035, 270.488)).

Conclusion: Locality significantly affects mental health status among youth in Malaysia. Therefore, it is suggested to also include a demographic aspect in considering any preventive measures about this issue. However, a larger scale of study (currently ongoing) is much needed to generalise the findings among Malaysian youth. 
Poster Abstract 35

Fracture risk (FRAX) and fall risk (FRAT) assessment in the elderly. should we assess both to determine fracture risk?

Yusaini $\mathrm{Y}^{1}$, Farnaza $\mathbf{A}^{1}$, Nafiza $\mathrm{MN}^{1}$, Mohd Rodi $\mathrm{I}^{2}$

${ }^{1}$ Primary Care Medicine, Faculty of Medicine, Selayang Campus, Universiti Teknologi MARA, 68100 Selangor; ${ }^{2}$ Public Health and Population Medicine, Faculty of Medicine, Sungai Buloh Campus, Universiti Teknologi MARA, 47000 Selangor

Introduction: The elderly are at risk of falls. When an elderly with osteoporosis falls, this can lead to fracture and further complications. FRAX tool is commonly used in primary care to assess fracture risk. There is conflicting evidence that proves FRAX calculation represents falls risk, therefore, this study intends to examine the agreement between the FRAX and FRAT (falls risk) score, and factors associated with fracture risk. Methods: This was a cross-sectional study involving elderly patients aged 60 years and above who attended a specialist primary care clinic. The study was conducted using a questionnaire that consists of sociodemographic and clinical characteristics, and the FRAX and FRAT assessment tool. Data were entered and analysed using SPSS version 23.

Results: A total of 307 participants were analysed with a mean age \pm SD of $68.08 \pm 6.05$. The prevalence for high fracture risk and high fall risk was $23.5 \%$ (CI: 18.7, 28.2) and $26.7 \%(95 \%$ CI: $21.7,31.7)$ respectively. Six factors were found to be associated with high fracture risk and they were higher age group $(\mathrm{p}<0.001)$, non-Malays $(\mathrm{p}<0.001)$, lower BMI $(\mathrm{p}<0.001)$, history of previous fracture $(\mathrm{p}<0.001)$, no past medical history of gout $(\mathrm{p}=0.021)$ and patient on calcium supplement $(p=0.026)$. The study found that there was no agreement between FRAX and FRAT scores.

Conclusion: FRAX score does not determine fall risk among the elderly. Therefore, it is important to assess both fracture risk and fall risk to ensure that elderly with high fall risk will also receive appropriate prevention and intervention.

\section{Poster Abstract 36}

Development and validation of the Malay self-efficacy, knowledge, attitude and avoidance practice towards environmental tobacco smoke questionnaire (SE-KAPETSQ) for mothers with children below 6 years old

Ahmad Muslim AR ${ }^{1}$, Farnaza $\mathbf{A}^{\mathbf{1}}$, Mohamad Rodi $\mathrm{I}^{2}$, Punithavathy $S^{3}$

${ }^{1}$ Primary Care Medicine, Faculty of Medicine, Universiti Teknologi MARA (UiTM), Jalan Prima Selayang 7, 68100 Batu Caves, Selangor, Malaysia; ${ }^{2}$ Public Health \& Population Medicine, Faculty of Medicine, Universiti Teknologi MARA (UiTM), Jalan Hospital, 47000 Sungai Buloh, Selangor, Malaysia; ${ }^{3}$ School of Medicine, Faculty of Health \& Medical Sciences, Taylor's University, Subang Jaya, Selangor, 47500 Malaysia.

Introduction: Children under 6-years old are most susceptible to environmental tobacco smoke (ETS). Mothers spend more time with their children and need to be empowered to protect their children from ETS. The study aimed to develop and validate a Malay ETS questionnaire that measures the self-efficacy, knowledge, attitude, and avoidance practice (SE-KAP-ETSQ) among mothers with children below 6 years old.

Methods: The SE-KAP-ETSQ was adapted from two questionnaires: 10-item EEQ and 29-item KAP-ETSQ (Malay). The two phases of the study are phase 1: development of the questionnaire and phase 2: validation of the questionnaire. Data were analysed using SPSS ver.26 and AMOS ver.24.

Results: A total of 513 mothers; (186 for Exploratory factor analysis (EFA) and 327 for Confirmatory factor analysis (CFA)) responded with a mean age of 31.44 years. EFA identified four domains with 26 items. Factor loadings ranged from 0.574 to 0.854 and Cronbach's alpha ranged from 0.755 to 0.887 . CFA generated four models before it achieved best-fit indices with root mean square error of approximation (RMSEA) of 0.053, goodness-of-fit index (GFI) was 0.932, comparative fit index (CFI) was 0.968, Tucker-Lewis index (TLI) was 0.960, Chi-squared/degree of freedom $\left(\chi^{2} / \mathrm{df}\right)$ was 1.909 . This produced 17 items with factor loadings ranged from 0.614 to 0.948 . Composite reliability and average variance extracted of the domains ranged from 0.778 to 0.858 and 0.534 to 0.689 , respectively. Conclusion: This study produced a valid and reliable new Malay SE-KAP-ETSQ that can be used for further studies within a wider population.

\section{Poster Abstract 37}

Perceived barrier towards Hepatitis $\mathrm{C}$ management among Primary Care Doctors in Sabah

\section{Emilina PT Tan ${ }^{1}$, Teh Rohaila $\mathbf{J}^{2}$, Raja Affendi RA ${ }^{3}$}

${ }^{1}$ Klinik Kesihatan Menggatal, Kota Kinabalu, Sabah, Kementerian Kesihatan Malaysia; '2Department of Family Medicine, Faculty of Medicine, Universiti Kebangsaan Malaysia, tehrohaila@ppukm.ukm.edu.my; ${ }^{3}$ Gastroenterology,Department of Medicine, Faculty of Medicine, Universiti Kebangsaan Malaysia, Bandar Tun Razak,draffendi@ppukm.ukm.edu.my

Introduction: Recent changes in the Malaysian healthcare system paves the way for doctors to manage hepatitis $\mathrm{C}$ virus (HCV) infection in government primary care clinics. The aim of this study was to evaluate the perceived barrier towards the management of hepatitis $\mathrm{C}$ among primary care doctors in Sabah, Malaysia.

Methods: A cross-sectional study involving 224 primary care doctors working in government primary care clinics throughout Sabah, Malaysia. Data was collected using universal sampling via online questionnaire using Google Form. Participant's socio-demographic, practice characteristics and perceived barriers (10 items) towards management of $\mathrm{HCV}$ was collected using an English language questionnaire.

Results: The participants were mainly female (64.7\%), 
medical officers $(90.2 \%)$, working in health clinics $(85.7 \%)$ with mean (SD) of 6.0(4.6) years of practice. Less exposure on HCV treatment $(95.5 \%)$, inadequate training $(92.4 \%)$ and inadequate knowledge (86.2\%) were identified as the main perceived barriers towards the management of HCV. While no doctor available in certain government primary care clinic (37.9\%), poor referral systems (41.5\%) and expensive medication $(60.7 \%)$ were the least reported perceived barrier in management of $\mathrm{HCV}$.

Conclusion: The main barriers were less exposure and inadequate knowledge. Further training and updates on $\mathrm{HCV}$ are needed for primary care doctors with particular emphasis on treatment to prepare them in managing hepatitis $\mathrm{C}$ in primary care.

\section{Poster Abstract 38}

\section{Audit on Optimisation of Diabetic Control Over 5 Year Period (2016-2020) in Menglembu Health Clinic in Kinta District}

\author{
Marian $\mathbf{N}^{1}$, Prasintia $\mathrm{P}^{1}$, Phan $\mathrm{AP}^{1}$, Asmah $\mathrm{ZA}^{2}$ \\ ${ }^{1}$ Menglembu Health Clinic, Perak; ${ }^{2}$ Kinta District Health \\ Office, Perak
}

Introduction: As part of the Ministry of Health (MOH) quality assurance program for diabetes, the Diabetes Clinical Audit is conducted annually. This includes the percentage of diabetic patients achieving HbA1c targets $<6.5 \%$. Targeted KPI by the Ministry of Health (MOH) is $30 \%$. The objective of the study is to identify the percentage of optimised HbAlc targets achieved and the contributing strategies from the year 2016-2020 in a health clinic in Kinta district.

Methods: A retrospective cohort study using quantitative data was used. Random sampling was taken from diabetic patients registered under National Diabetic Registry (NDR) from 2016 to 2020. The sampling for annual audit is determined by the state health department whereby deceased and transferred out patients are excluded.

Results: The percentage of optimal HbA1C targets achieved from the year 2016-2020 were 38\%, 37.4\%, 39.6\%, 32\% and $43.1 \%$ respectively. Strategies identified which contributed to this achievement include a dedicated and systematic management of diabetes programmes, comprehensive clinical management and empowerment of patients.

Conclusion: KPI target of $30 \%$ for $\mathrm{HbA} 1 \mathrm{C}<6.5$ percent was achieved over the past 5 years by implementing strategies involving a multidisciplinary approach.

\section{Poster Abstract 39}

Insulin injection knowledge and practices among diabetic patients in UITM Primary Care Specialist Clinic

\section{Siti Fatimah BS ${ }^{1}$, Sharina $\mathrm{J}^{1}$, Fareesya $\mathrm{HMFi}^{1}$, Asma $\mathrm{AG}^{1}$ ${ }^{1}$ Department of Primary Care Medicine, Faculty of Medicine, Universiti Teknologi MARA}

Introduction: Insulin is widely used in the management of Diabetes mellitus (DM). Correct insulin injection knowledge and practices are important in achieving glycemic control and prevention of complications. This study aimed to assess the insulin injection knowledge and practices among diabetic patients on insulin attending UiTM Primary Care Specialist clinics in Selayang \& Sg Buloh Campus.

Methods: A cross sectional study was done on DM patients who attended the clinic for their follow up between 1st - 21st July 2019. Their insulin injection knowledge and practices were assessed via face-to-face interview.

Results: A total of 45 patients were recruited. The mean age of the patients was 62.5 years old $(S D \pm 9.21)$. Majority were male (55.6\%), Malay (84.4\%), has had DM for more than 10 years $(73.3 \%)$ with $40 \%$ of them having 4 insulin injections per day. Most of the patients had correct knowledge of insulin storage (94\%), however less than half knew that opened pens must be used within 30 days (44.4\%). Majority of patients correctly; 1) placed a needle onto the pen $(97.8 \%), 2$ ) dialed the desired dose (95.6\%), 3) chose a suitable site for injection $(91.1 \%), 4)$ inserted insulin needle into the skin $(93.3 \%), 5)$ pressed insulin pen plunger $(100 \%)$, 6) counted for dwell time before withdrawing needle $(71.1 \%), 7)$ disposed used needle safely (100\%), 8) rotated site of injection (97\%) and 9) injected insulin at the time gap prior to meals.

Conclusion: The findings of this study highlights that our patients had an overall good insulin injection knowledge and practice, however there is still room for improvement in the delivery of education for patients.

\section{Poster Abstract 40}

Clinical audit on management for type 2 diabetes mellitus patients in UITM Primary Care Specialist Clinic, Selayang campus

Nik Aminah NAK, Nasturah A, Nurul Izzah S, Hakimah KS. Siti Fatimah BS

Department of Primary Care Medicine, Faculty of Medicine, Universiti Teknologi MARA

Introduction: Type 2 Diabetes Mellitus (T2DM) is a major non-communicable disease and its management in primary care is important. This audit aimed to assess the quality of care in the management of T2DM patients at UiTM Primary Care Specialist Clinic, Selayang Campus.

Methods: A retrospective audit of electronic medical records was conducted between 1st January until 31st December 2019. Thirty-one criteria were set, and the standards were determined based on the national T2DM clinical practice guideline (CPG).

Results: A total of 537 medical records were audited; 21 from 31 criteria achieved the standard. This includes 1) availability of a multidisciplinary team managing T2DM, 2) selfmanagement booklets, 3) CPG in each consultation room, 4) facilities for performing laboratory investigations, 5) 12-lead ECG, 6) fundoscopy, 7) family history of premature CVD, 8) smoking status, 9) documentation of blood pressure, 10) body mass index at every visit, 11) monitoring of HbAlc and 12) serum lipid profile at least 3-6 monthly, 13) performing renal 
function test, 14) liver function test and 15) urinalysis / UACR at least once in a year, 16) advising on physical activity, 17) referral of patients with diabetes to a dietitian or given dietary advice, 18) initiation of statins at the age $>40$ (unless contraindicated), 19) prescription of ACE-I as first-line for patients with hypertension, 20) patients who achieved individualized HbA1c target and 21) patients who achieved LDL target.

Conclusion: The current audit findings showed an overall improvement compared to a previous audit done in this centre in 2019. Comprehensive strategies are required for improvement of delivery of care and implementing recommendations from this audit.

\section{Poster Abstract 41}

\section{Hajj health examination for pilgrims with asthma in Malaysia: An ethnographic study}

Rizawati $\mathbf{R}^{1}$, SM Liew ${ }^{1}$, EM Khoo ${ }^{1}$, Nik Sherina $\mathrm{H}^{1}$, Norita $\mathrm{H}^{1}$, PY Lee 2 , Sazlina SG ${ }^{2}$, ATCheong ${ }^{2}$, Ahmad Ihsan $\mathrm{AB}^{3}$, Azah $\mathrm{AS}^{4}$, Suhazeli $A^{4}$, H Pinnock ${ }^{5}$, Aziz $S^{5}$, on behalf of the RESPIRE Collaborators $^{4}$

${ }^{1}$ Department of Primary Care Medicine, Faculty of Medicine, University of Malaya, Malaysia; ${ }^{2}$ Faculty of Medicine and Health Sciences, University Putra Malaysia, Malaysia; ${ }^{3}$ Hospital Pusrawi Pvt Ltd; ${ }^{4}$ Ministry of Health, Malaysia; ${ }^{5}$ Usher Institute, University of Edinburgh, United Kingdom

Introduction: To observe current organisational and clinical routines of Hajj health examination in Malaysia with a focus on the delivery of care for pilgrims with asthma.

Methods: We conducted non-participant observation to obtain ethnographic understanding of Hajj health examination activities for 2019. Observations were guided by a checklist and recorded as notes that were analysed thematically. The study was conducted at 12 public and two private primary care clinics.

Results: We observed considerable variations in the implementation and practice of Hajj health examinations among the 12 public clinics but no marked variation among the private clinics. The short time span of between three to four months from the issuance of Hajj offer to departure put pressure on health care providers. They mostly regarded the Hajj health examination as merely a certification of fitness to conduct the pilgrimage, though respiratory health assessment was often inadequate. The opportunity to optimise health of the pilgrims with asthma by providing the appropriate medications, asthma action plan and education on preventive measures was disregarded. The preliminary health screening, which aimed to optimise pilgrims' health before the Hajj health examination was not appreciated by either pilgrims or health care providers.

Conclusion: There is great potential to reform the Hajj health certification process and to optimise its potential benefits for pilgrims with asthma. A systematic approach to restructuring the delivery of Hajj health examination could address the time constraints and huge volume of pilgrims to reduce the risk posed from asthma and other long-term conditions during pilgrimage.
Poster Abstract 42

\section{Case report: Trigeminal Herpes Zoster post COVID-19} vaccination. Is there an association?

Anuar S*, Mohd Radzniwan A R, Khairun Nain NA, Khairullah A, Fadlul A, Fauzi M,, Faizul HA, Nizam B Faculty of Medicine \& Health Sciences, Universiti Sains Islam Malaysia, Malaysia

Introduction: Effective vaccination programme is critical to end the COVID-19 pandemic. Emerging cases reported as adverse event following immunisation are concerning. This is a case report of trigeminal herpes zoster (HZ) that occurred following COVID-19 vaccination which raised questions on it being an adverse effect or merely coincidental.

Case Report: A 56-year-old man received 2 doses of PfizerBioNTech $^{\text {TM }}$ COVID-19 vaccine on the 25 th of June and 16th of July 2021. With a history of well controlled hypertension, he presented to his general practitioner (GP) with severe pain over his right frontal-temporal area, including buccal cavity and molar area a day prior to his second dose of PfizerBioNTech vaccine. He was treated as gingival infection as there was history of a right incisor tooth extraction during the previous week. Despite the pain, he completed his second dose of COVID-19 vaccine on the next day. However, the pain increased, and he saw his GP again 10 days later with a diagnosis of atypical migraine. Finally, on 29th of July, vesicular rash erupted, and the diagnosis of Trigeminal $\mathrm{HZ}$ was made.

Conclusion: $\mathrm{HZ}$ is common with the estimated incidence of 3.4-4.82 per 1000 person years. However, there are anecdotal reports of an increasing HZ emergence post COVID-19 vaccination. Nonetheless, there is no evidence of this causation. It could occur anytime as the lifetime risk is in between 25\% and 30\% thus it can erupt naturally. Further studies are needed to determine the association between COVID-19 vaccine and $\mathrm{HZ}$ emergence.

\section{Poster Abstract 43}

\section{Adolescents' Diet Quality at Home Food Environment: A Qualitative from mothers' perspective.}

\section{Suhaila AG1,2, Norimah A K3, Ruzita AT1, Noor Ani A2, Ahmad AZ2 \\ ${ }^{1}$ Nutritional Science Programme, Faculty of Health Science, Universiti Kebangsaan Malaysia, Kuala Lumpur; ${ }^{2}$ Institute for Public Health Malaysia, Ministry of Health, Setia Alam, Selangor; ${ }^{3}$ International Medical University, Bukit Jalil, Kuala Lumpur}

Introduction: The home food environment influences adolescents' eating behaviour and potentially affects overall diet quality. Unfavorable home food environment may contribute to higher prevalence of obesity among adolescents. This study aims to explore qualitatively any perceived factors related to adolescents' diet quality at home from parents' perspective.

Methods: A qualitative study based on Socio Ecological Model purposively sampled twenty-seven multi-ethnic mothers 
from four regions in Malaysia [Southern (Johor), Central (Selangor), Northen (Kedah) and East Coast (Terengganu)]. This study utilized in-depth interviews, which were audiorecorded and fully transcribed and analyzed using Atlas.Ti 8.0 software. The transcribed data were grouped into several themes and sub-themes which were then categorised under different factors such as individual, social and environmental. Results: The results revealed that multifaceted factors at home environment contributed to unfavourable patterns of diet quality among adolescents. The availability and convenience of high dense snacks at home, accessibility to food outlets outside of home, adolescents' food preferences and time constraint due to parents' work commitments were identified as the main barriers towards healthy eating practice among adolescents. The results suggest that availability and accessibility to unhealthy food option at home which lead to snacking practice and tendency in meal skipping were associated with poor diet quality among adolescents.

Conclusion: Therefore, the findings will help to facilitate and design family-based obesity intervention that can raise awareness and knowledge among mothers and adolescents.

\section{Poster Abstract 44}

\section{Vesicular Rash Post COVID-19 Vaccination. Is It Varicella} Infection Related Or Side Effect?

\section{Mohd Radzniwan $\mathbf{A R}^{1,2}$, Sharifah Najwa $\mathrm{SM}^{1,2}$, Rokiah $S^{3}$} Anu Suria $\mathrm{G}^{1,2}$

${ }^{1}$ Department of Primary Healthcare, Faculty of Medicine and Health Sciences, Universiti Sains Islam Malaysia,Bandar Baru Nilai,71800, Nilai), Negeri Sembilan, Malaysia; ${ }^{2}$ Klinik Pakar Kesihatan USIM, Lot 193-194,6,Jalan Nilai Square 2,Bandar Baru Nilai,71800 Nilai,Negeri Sembilan; ${ }^{3}$ Poliklinik Kesihatan USIM, Lot 193-194,6,Jalan Nilai Square 2,Bandar Baru Nilai,71800 Nilai,Negeri Sembilan

Introduction: COVID-19 has caused significant health turbulence across the world. At the same time, the pandemic is to be combated with ongoing vaccination programs. Many have reported side effects from the vaccine. This case illustrates a case of a vesicular rash appearing post vaccination that presented a diagnostic challenge to her general practitioner (GP)

Case Report: A 25-year-old immunocompetent woman presented with low grade fever, itchy and painful vesicular rash, but not localised to any dermatome, 3 days post her first dose Pfizer-BioNTech vaccination. There was no history of exposure to a recent varicella infection, nor was any varicella vaccine taken prior. Doubtful of diagnosis, a herpes serology was sent and reported as negative for varicella $\operatorname{IgM}$ and borderline for IgG. She later received her second dose, to complete her Covid-19 vaccination, but similar characteristics rash appeared. Adverse event following immunisation (AEFI) was reported subsequently. Fortunately, the rashes resolved spontaneously with symptomatic treatment.

Conclusion: The fact that the rash was vesicular in nature had led her GP to rule out varicella infection at first presentation. However, because the similar rash appeared immediately post-second dose of Pfizer-BioNTech vaccination the diagnosis of varicella infection became less likely, supported by negative IgM herpes serology. Previous studies had reported the Moderna vaccine was associated with vesicular rash, but none were reported for Pfizer-BioNTech. Therefore, it is prudent to report it is an AEFI so that more side effect information can be added into this vaccine type.

\section{Poster Abstract 45}

Don't Underestimate Exudates: Management of a Traumatic Wound with Heavy Exudates.

Rachael SD ${ }^{1}$, Valli $\mathrm{R}^{1}$, Khalid $\mathrm{H}^{1}$

${ }^{1}$ Klinik Kesihatan Bandar Tasek Mutiara, Seberang Perai Selatan, Pulau Pinang

Introduction: Wound exudate or fluid can be described as nature's way of helping to heal the wound. With the correct moisture balance wound healing can be achieved quickly. Excessive exudates are usually treated with moisture absorbent dressing for example hydrofibers and foams in primary care clinics depending on the level of exudates present. This case report will describe the management of a heavily exudating traumatic wound using Negative Pressure Wound Therapy

Case Report: A 19-year-old young man presented with a non-healing post traumatic ulcer measuring $11.5 \mathrm{~cm} \mathrm{x} 8 \mathrm{~cm}$ x $1.5 \mathrm{~cm}$. Surgical debridement was done twice. Medical grade honey (Activon ${ }^{\oplus}$ Manuka Honey) was applied to the wound for osmotic debridement as well .Hydrofiber dressing Aquacel $\mathrm{Ag}^{\oplus}{ }^{\oplus} \mathrm{Hydrofiber}^{\ominus}$ dressing (ConvaTec) was later applied to control the exudate and slough. The wound remained heavily exudative so 3 cycles of Negative Pressure Wound Therapy (NPWT) ACTIV.A.C ${ }^{\text {Tm} T h e r a p y ~ S y s t e m ~ w a s ~ a p p l i e d . ~ T h e ~}$ exudates markedly improved and wound closure was achieved. Conclusion: Effective exudate management is important for wound healing. Recognizing the problem early is essential as it will also improve healing time.

\section{Poster Abstract 46}

Enhancing Healthcare Utilisation for the Asnaf During COVID-19 Pandemic: USIM Specialist Clinics Initiatives

Sharifah Najwa SM ${ }^{1,3}$, Mohd Radzniwan AR ${ }^{1,3}$, Fathima Begum SM ${ }^{1,3}$, Anu Suria $G^{1,3}$, Yafizah $Y^{1,3}$, Asfizahrasby MR MR $^{2,4}$ ${ }^{1}$ Department of Primary Healthcare, Faculty of Medicine and Health Sciences, Universiti Sains Islam Malaysia (USIM); ${ }^{2}$ Department of Surgery, Pathology and Medicine, Faculty of Dentistry, USIM; ${ }^{3}$ Klinik Pakar Kesihatan USIM, Bandar Baru Nilai, Negeri Sembilan.4Klinik Pakar Pergigian USIM, Bandar Baru Nilai, Negeri Sembilan

Introduction: Healthcare utilisation of the less fortunate is hampered by the COVID-19 pandemic. Hence, a health campaign jointly led by the Family Medicine and Dental Specialists was initiated at USIM's private specialist clinic to serve the asnaf. The campaign aims to provide specialised health care to asnaf in Nilai, Negeri Sembilan, through the difficult time of the COVID-19 pandemic. 
Methods: A clinic-based campaign for the asnaf was conducted every Thursday between February to July 2021. A total of RM20,000 from the Tabarru' drive was allocated for medical and dental expenses, including coverage for transportation. Family Medicine and Dental Specialists jointly saw all patients on the same day. Referrals to other disciplines were made when deemed necessary. Only referrals to the USIM Medical and Dental Specialists Clinic were covered by the campaign.

Results: Thirty-two individuals (65\% females, 35\% males) participated over the six months. Most of the medical problems seen were chronic disease-related, where $6 \%$ were cancer related. Personalised care and satisfaction were highlighted throughout this campaign. Compromised psychosocial circumstances of the asnaf and their family members were alleviated through free medical and dental services, plus subsidised logistics. The asnaf became more motivated to focus on their well-being.

Conclusion: This campaign builds new knowledge, awareness and self-efficacy among the asnaf. Such campaigns should be carried out regularly to ensure improvement of healthcare utilisation and achievement of health equity regardless of socioeconomic background.

\section{Poster Abstract 47}

\section{A Prompt Treatment of Renal Cell Carcinoma: The Coalition of The Masseuse, Primary Care Physician, Radiologist and Surgeon}

\author{
Sharifah Najwa SM ${ }^{1,4}$, Siti Soraya $\mathrm{AR}^{3,4}$, Rokiah ${ }^{5}$, \\ Ruhi Fadzlyana J,4 \\ ${ }^{1}$ Department of Primary Healthcare, Faculty of Medicine and \\ Health Sciences, Universiti Sains Islam Malaysia (USIM); \\ ${ }^{2}$ Department of Surgery, Faculty of Medicine and Health \\ Sciences, USIM; ${ }^{3}$ Department of Radiology, Faculty of Medicine \\ and Health Sciences, USIM; ${ }^{4}$ Klinik Pakar Kesihatan USIM, \\ Bandar Baru Nilai, Negeri Sembilan; 5 Poliklinik Kesihatan \\ USIM, Bandar Baru Nilai, Negeri Sembilan
}

Introduction: The primary care visit due to non-coronavirus related clinical presentation have drastically reduced throughout the world during COVID-19 pandemic. We illustrate a prompt detection of Renal Cell Carcinoma (RCC) in a patient presenting with a hidden agenda to a primary care clinic, in the midst of this pandemic.

Case Report: A 40-year-old lady without prior medical problems, sought a second opinion at our centre regarding her normal blood and urine investigation results. During the clinical consultation, she was concerned about her right sided abdominal discomfort and mass that was first noticed by her masseuse few weeks prior to presentation. Abdominal examination revealed a ballotable right upper quadrant mass, measuring $6 \times 5 \mathrm{~cm} 2$. Prompt referral for abdominal ultrasound revealed a right mid and lower pole solid renal mass with cystic components within. A four-phase renal and staging computed tomography (CT) demonstrated features suggestive of RCC with no evidence of metastasis.
A laparoscopic nephrectomy was done one week later with final histological diagnosis of clear cell carcinoma, staged T3N0M0.

RCC presentations are often asymptomatic making early diagnosis challenging. Role of surgery and immunotherapy is well established in improving patient survival. Point of care imaging supervenes early diagnosis of abdominal masses. Traditional and complementary medicine is part of the cultural heritage and belief system with its own inherent value and efficacy for health promotion, prevention and treatment in Malaysia.

Conclusion: The primary healthcare role in ensuring timely referral to the specific specialty ensues a comprehensive patient care especially during COVID-19 pandemic.

\section{Poster Abstract 48}

Prevalence of Primary Dysmenorrhea and Factors Associated Among Women at Kuala Selangor - A CrossSectional Study

Yafizah $\mathbf{Y}^{1}$, Adibah $\mathrm{HI}^{2}$, Nurainul $\mathrm{HS}^{2}$, Anu Suria $\mathrm{G}^{1}$, Fathima BSM ${ }^{1}$

${ }^{1}$ Family Medicine Unit, Faculty of Medicine and Health Sciences, Universiti Sains Islam Malaysia; ${ }^{2}$ Department of Family Medicine, Faculty of Medicine and Health Sciences, Universiti Putra Malaysia

Introduction: Primary dysmenorrhea (PD) is one of the womanhood gynaecological problems and affects daily living activity. This study aims to determine prevalence, severity and factors associated with PD at Kuala Selangor, Selangor.

Methods: A cross-sectional study with systematic random sampling was carried out from July until September 2017 among 215 women, aged 18-35 years old at Kuala Selangor Health Clinic, Selangor. Structured and validated questionnaires were develop covering sociodemographic and lifestyle components. Data were analyzed into frequency and percentage, and multiple logistic regression was performed for the statistically significant association.

Results: 210 women with a response rate of $98.6 \%$ participated in this study. PD prevalence was $60.5 \%$ with pain severity were $13.4 \%, 75.6 \%$ and $11.0 \%$ for mild, moderate and severe. Significant factors associated with PD were nulliparous (OR: 5.1, CI: 1.508, 17.277, p: 0.009), first degree family history of dysmenorrhea (OR: 4.431, CI: $1.727,11,368$, p: 0.002 ) and lack of regular physical exercise (OR: 14.037, CI: 5.161, 38.183, p: <0.001). However, there were no significant associations between age, race, occupation, body mass index, attempting to lose weight, smoking, second-hand smoker and frequent fast-food intake with PD.

Conclusion: The prevalence of PD is still high which majority of them had moderate pain. The health care provider should emphasize women to be more active in practicing regular physical exercise. Hence, future study in multiple places with bigger sample size is warranted to look for possible lifestyle factors contribute to PD. 
Poster Abstract 49

\section{Neglecting child neglect: A forgotten form of child} maltreatment

\section{Chow SY}

${ }^{1}$ Klinik Kesihatan Taman Medan, Selangor, Malaysia

Introduction: Child maltreatment is defined as any acts of commission or omission by a parent or other caregiver that result in harm, potential harm, or threat of harm to a child, even if it is not the intended result. There are four forms of maltreatment: physical abuse, sexual abuse, emotional abuse and neglect. Child neglect is very often not well recognized and receives the least attention.

Methods: Two cases of child neglect seen at primary care were described.

Results: The first case was a day 3 of life term baby boy who was referred from Hospital $S$ to the primary care clinic for evaluation of neonatal jaundice. The parents had refused for any assessment and intervention despite the baby noted jaundiced till the soles of the feet. Despite counselling, the parents refused to seek treatment. The second case was a mother with major depression disorder with psychosis. The father was rarely at home. Both parents were also occasionally arguing in front of their children. Their children were exposed to this volatile relationship and experienced supervisory and emotional neglect. Both cases were seen by the family physician. Child Act 2001 was used to manage these cases. Social welfare department were involved. Both cases ended with parents addressing their children's needs.

Conclusion: Child neglect is often under-reported. Primary care physicians play an important role in early recognition and by using an approach that combines the focus on child safety, and child and family welfare, the consequences of child neglect can be tackled.

\section{Poster Abstract 50}

\section{Modern contraceptive usage and its associated factors} among Orang Asli women attending community clinics in east Pahang.

\section{Nurjasmine $\mathrm{AJ}^{1}$, Muhammad Zul Hazmi AZ ${ }^{1}$, \\ Nor Azam $\mathrm{K}^{1}$, Hafizah $\mathrm{P}^{2}$ \\ ${ }^{1}$ Department of Family Medicine, Kulliyyah of Medicine, International Islamic University Malaysia; ${ }^{2}$ Department of Community Medicine, Kulliyyah of Medicine, International Islamic University Malaysia}

Introduction: There is high unmet need for modern contraception among Orang Asli women. This study aimed to measure the prevalence and associated factors for contraceptive usage among Orang Asli women in east Pahang.

Methods: A cross-sectional study was conducted from August to December 2020 at four selected health clinics in Kuantan, Pekan and Rompin, Pahang. A validated questionnaire was used to assess the contraceptive usage. A total of 217 Orang Asli women who fulfil the inclusion criteria were included. Descriptive analysis was used to measure the prevalence using SPSS version 26.0. Association between all independent variables with contraceptive usages were analysed using the Chi square test.

Results: The prevalence of modern contraceptive usage was $88.9 \%$. The most common reported contraceptive methods was OCP (56.2\%), followed by IM depoprovera (43.3\%\%), male condom and implanon. Our result showed that parity $\left(\chi^{2}=4.514, \mathrm{df}=1, \mathrm{p}=0.034\right)$, obstetric complications $\left(\chi^{2}\right.$ $=4.317, \mathrm{df}=1, \mathrm{p}=0.038)$, exposure on information regarding contraception $\left(\chi^{2}=8.725, \mathrm{df}=1, \mathrm{p}=0.003\right.$, household income $\left(\chi^{2}=6.906, \mathrm{df}=1, \mathrm{p}=0.009\right)$ and timing of first contraceptive usage $\left(\chi^{2}=48.351, \mathrm{df}=1, \mathrm{p}=0.003\right)$ were associated with contraceptive usage.

Conclusion: Modern contraceptive usage among Orang Asli women in East Pahang was high, with OCP been the most popular contraceptive methods used. Factors associated with contraceptive usage were parity, household income, obstetric complications, timing of first contraception usage and exposure on information regarding contraception. Clinician should tirelessly promote the contraceptive usage among Orang Asli female with comorbidity, aiming to preserve excellent women health among this population.

\section{Poster Abstract 51 \\ Knowledge, attitude, and confidence in dementia management among primary care doctors in Malaysia - the importance of postgraduate training}

Nurul Izzah $S^{1}$, Mohamed Syarif $\mathbf{M Y}{ }^{1 \S}$, Mariam $\mathbf{M}^{2}$, Noorhida B ${ }^{1}$

${ }^{1}$ Department of Primary Care Medicine, Faculty of Medicine, Universiti Teknologi MARA, ${ }^{2}$ Department of Public Health Medicine, Faculty of Medicine, Universiti Teknologi MARA

Introduction: Primary care doctors (PCDs) play an important role in the timely diagnosis and management of dementia. This study aimed to determine the knowledge, attitude, and confidence (KAC) in managing dementia among PCDs with postgraduate qualification (PCD-PG-Qual) and without postgraduate qualification (PCD-noPG-Qual) in Malaysia.

Methods: A cross-sectional study was conducted via an online questionnaire (GoogleForms). It involved PCDs with $\geq$ one-year working experience in Malaysian primary care settings, who were members of the Family Medicine Specialists Association (FMSA), Academy of Family Physicians Malaysia (AFPM), or Malaysian Primary Care Network (MPCN) Facebook group. Invitations were sent by committee members via Whatsapp ${ }^{\oplus}$ (FMSA), email (AFPM), and Facebook posting (MPCN). Sociodemographic and work-related characteristic data were collected. Knowledge, attitude, and confidence in managing dementia were determined using the Dementia Knowledge among General Practitioners' questionnaire and General Practitioners' attitude and confidence scale for dementia. Independent t-test was used to compare the mean KAC scores between PCD-PG-Qual and PCD-noPG-Qual. Results: Out of 270 respondents, 239 fulfilled the inclusion and exclusion criteria and completed the questionnaire. The 
mean age was $36.7 \pm 8.6$ years old. The majority were females (72.4\%) and Malay (64.4\%). 102 (42.7\%) had postgraduate qualifications in Family Medicine. Mean knowledge score was significantly higher in PCD-PG-Qual compared to PCDnoPG-Qual (12.34, $\mathrm{SD} \pm 3.35$ vs. 8.69, $\mathrm{SD} \pm 3.90), \mathrm{t}(232)=$ 7.77, $\mathrm{p}<0.001$. Mean confidence score was also significantly higher in PCD-PG-Qual compared to PCD-noPG-Qual (3.28, $\mathrm{SD} \pm 0.63$ vs. $2.73, \mathrm{SD} \pm 0.77), \mathrm{t}(235)=6.08, \mathrm{p}<$ 0.001 . However, difference between their mean attitude scores was not statistically significant: PCD-PG-Qual vs. PCDnoPG-Qual (4.34, $\mathrm{SD} \pm 0.42$ vs. 4.27, $\mathrm{SD} \pm 0.40), \mathrm{t}(237)=$ $1.47, \mathrm{p}=0.143$.

Conclusion: PCDs with postgraduate qualifications had better knowledge and confidence in managing dementia. This underlines the pertinence of professional training and certification.

\section{Poster Abstract 52}

\section{Attitudes of primary care doctors on depression: a cross sectional study in Terengganu}

Nurul Izza $\mathbf{Y}^{1}$, Nur Aiza $I^{1}$, Nurul Azreen $Y^{1}$, Nur Baiti $Y^{2}$, Norimah $\mathrm{R}^{3}$, Nur Fatiha $\mathrm{K}^{4}$, Mohammad Hilfi $\mathrm{R}^{5}$, Adina $\mathrm{A}^{6}$ ${ }^{1}$ Family Medicine Unit, Faculty of Medicine, Universiti Sultan Zainal Abidin, Terengganu; ${ }^{2}$ Klinik Kesihatan Kuala Kemaman, Ministry of Health Malaysia, Terengganu,Malaysia; ${ }^{3}$ Pejabat Kesihatan Cheras, Ministry of Health Malaysia, Wilayah Persekutuan Kuala Lumpur, Malaysia; ${ }^{4}$ Klinik Kesihatan Kuala Dungun, Ministry of Health Malaysia, Terengganu, Malaysia; ${ }^{5}$ Klinik Kesihatan Bentong, Ministry of Health Malaysia, Pahang, Malaysia; 'Department of Primary Care Medicine, Faculty of Medicine, University of Malaya, Kuala Lumpur

Introduction: Depression is a common mental disorder associated with substantial disability. Moving towards community-oriented management of mental health problems, primary care doctors play a vital role in screening, identifying, and managing common mental health problems. This study aimed to examine the primary care doctor's attitude towards depression and determine its association with their sociodemographic factors, training background, and clinical experience.

Methods: A cross-sectional study is done between March to April 2018 amongst 144 government primary health care medical officers working in health clinics in Terengganu. The Revised Depression Attitude Questionnaire (R-DAQ), a validated 22 -items Likert scale (0-5), was used to determine their attitude towards depression and its treatment in primary care. Respondents' sociodemographic factors and training experiences are collected using a self-administered questionnaire. The data was analyzed using the IBM SPSS statistics software version 19.0.

Results: The mean age of participants was 31 years old; majority were female (78.5\%) and Malay (94.4\%). The respondents demonstrate a good perspective about depression occurrence, recognition, and management $($ mean $=3.2)$. There is a significant lack of professional confidence in managing depression $($ mean $=1.4)$ and moderate pessimistic views on depression treatment outcomes (mean $=3.5)$. Doctors with a longer duration of clinical experience have more optimistic views about depression $(\mathrm{p}=0.013)$. In contrast, those with a personal history of depression and more frequent encounters with depressed patients have more pessimistic views ( $\mathrm{p}=$ 0.003, $\mathrm{p}=0.017$ ).

Conclusion: The study findings indicate the dire need for targeted training for primary care providers in managing common mental health problems.

\section{Poster Abstract 53}

\section{Development and validation of the Screen Dependency Scale (SDS) among pre-school children}

\author{
Azwanis $\mathrm{AH}^{1}$, Siti Ruziana $\mathrm{R}^{1}$, Edre $\mathrm{MA}^{2}$, Nurzulaikha $\mathrm{A}^{3}$ \\ ${ }^{1}$ Department of Family Medicine, International Islamic \\ University Malaysia; ${ }^{2}$ Department of Community Medicine, \\ International Islamic University Malaysia; ${ }^{3}$ Biostatistics and \\ Research Methodology Unit, School of Medical Sciences, \\ Universiti Sains Malaysia
}

Introduction: Excessive screen time in young children is associated with many harmful consequences including screen dependency. Research have shown worrying prevalence of media related dependency among adolescents but questionnaires among pre-school children specifically is lacking which explains the paucity of data in this group. This study aimed to develop and validate a questionnaire to assess screen dependency among pre-school children aged 4 to 6 years old.

Methods: A cross sectional 2-phase study was carried out. During Phase 1, a preliminary parent report measure questionnaire was developed in Bahasa Malaysia. It was sent to four experts for content validity followed by face validity. In Phase 2, a total of 386 parents of pre-school children aged 4 to 6 years old, split into two samples, were involved in the field study for exploratory factor analysis (EFA) and confirmatory factor analysis (CFA).

Results: Sample 1 was used to perform EFA to determine the factorial structure of the SDS. All items with factor loading of $>$ 0.4 were included. Sample 2 was used to perform the CFA and RMSEA, GFI and CFI analysis showed that the SDS has a good fit and confirms the dimensional structure found via EFA. The final questionnaire consists of 15 items with 4 factors structure and has an excellent internal consistency reliability of 0.899 .

Conclusion: The Screen Dependency Scale (SDS) is a reliable and valid questionnaire to detect screen dependency among preschool children ages 4 to 6 years old in Malaysia.

\section{Poster abstract 54}

Malay Mother's Views on Sexuality and Sexual Education to their Intellectual Disabilities Children (A Qualitative Study)

\footnotetext{
Nawal NK ${ }^{1}$, Rosediani $\mathrm{M}^{1}$, Zainab MY' ${ }^{2}$, Rosnani $\mathrm{Z}^{1}$ ${ }^{1}$ Department of Family Medicine, School of Medical Sciences, Universiti Sains Malaysia, Kampus Kesihatan, Kubang Kerian, Kelantan, Malaysia. 2School of Dental Sciences, Universiti Sains Malaysia, Kampus Kesihatan, Kubang Kerian, Kelantan, Malaysia.
} 
Introduction: Sexuality and sexual education of persons with disabilities are still misjudged and perceived negatively by society even though this group exhibits comparable normal sexual interest and desires to the general population. Sexual education is a taboo subject in Malay society, despite it is crucial in preventing the surge in sexual offenses against this group, which is particularly vulnerable. This study aims to explore Malay mother's views on sexuality and sexual education to their children with Intellectual Disabilities.

Methods: This qualitative study used in-depth interviews and a phenomenological approach. A semi-structured questionnaire was applied during the interview. Twenty mothers of children aged 10-19 who had mild to moderate intellectual disabilities or other cognitive disabilities (Autism Spectrum Disorder, Attention Deficit Hyperactivity Disorder, Down Syndrome) were recruited from volunteer groups in Kota Bharu, Kelantan. Results: Almost every mother acknowledges their children's emerging sexuality as they go through adolescence. However, how they confront sexuality and sexual education is different. The way mothers perceive sexuality in their children will influence the extent to which sexual education is provided. Three themes emerged from their experiences: Revealing the meaning of sexuality, realising the actions of sexual behaviour (realising sexual urge, uneasiness toward sexually related display and expecting of delay in non-physical sexual maturity), and selective sexual education.

Conclusion: This study emphasizes the need for programs that promote sexual awareness and provide applicable sexual education to children with disabilities and their parents to help them manage their children's emerging sexuality effectively.

\section{Poster abstract 55}

Knowledge, Attitude, Intention To Screen For Thalassaemia And Its Associated Factors Among Unmarried Individual Attending Premarital Course In Kota Bharu.

Muhammad Akmal MN'1, Maryam $\mathrm{MZ}^{2}$, Imran $\mathrm{A}^{2}$, Nur Suhaila I ${ }^{2}$

${ }^{1}$ Klinik Kesihatan Sindumin, 89850 Sipitang, Sabah; ${ }^{2}$ Department of Family Medicine, Universiti Sains Malaysia (Health Campus), Kubang Kerian, 15200 Kota Bharu, Kelantan

Introduction: In Malaysia, the prevalence of thalassaemia is high, with great burden to the patients and community. Screening for thalassaemia among unmarried individuals play a key role in preventing thalassaemia major births. It is important to assess these aspects among them so that specific health education can be given appropriately to them. Objectives were to determine the mean knowledge score of thalassaemia, to describe the attitude and intention to screen for thalassaemia among unmarried individuals in Kota Bharu, and to determine the associated factors for good knowledge of thalassaemia.

Methods: It was a cross sectional study conducted in May 2017 involving pre-marital course participants. The questionnaire used consists of four parts, general information of participants, knowledge of thalassaemia, attitude towards thalassaemia and intention to screen for thalassaemia.

Results: 278 respondents were involved in this study. Mean (SD) knowledge score for thalassaemia was 8.8 (4.99) out of possible score of 21 . The associated factor for good knowledge of thalassaemia using multiple linear regression was professional group. Most respondents agree that unmarried individuals should screen for thalassaemia before marriage, however only $5 \%$ of premarital course respondents have ever screened for thalassaemia. They also mostly disagree that that thalassaemia carriers should not marry and should not have children. Prenatal diagnosis and termination of pregnancy for babies diagnosed with thalassaemia major were also mostly disagreed.

Conclusion: The knowledge of thalassaemia among unmarried individuals in Kota Bharu is still low. Further health promotion and education regarding thalassaemia screening is needed and tailored for them.

\section{Poster Abstract 56}

Factors Associated with High-Sensitivity C-Reactive Protein and Interleukin-6 among Adults with Metabolic Syndrome in a Primary Care Clinic

Jazlan $\mathbf{J}^{1}$, Suraya $\mathrm{AR}^{1,2,3}$, Zaliha I ${ }^{4}$, Siti Hamimah $S A K^{3,5,6}$, Danial EG ${ }^{5,6}$, Thuhairah $\mathrm{HAR}^{7}$, Nur Amirah $\mathrm{Si}^{1}$, Nur Atiqah $\mathrm{AR}^{7}$

1Department of Primary Care Medicine, Faculty of Medicine, Universiti Teknologi MARA, Jalan Prima Selayang 7, Batu Caves, Selangor, Malaysia; 2Cardio Vascular and Lungs Research Institute (CaVaLRI), Hospital Universiti Teknologi MARA (HUiTM), Jalan Hospital, Sungai Buloh, Selangor, Malaysia; 3Institute for Pathology, Laboratory and Forensic Medicine (I-PPerForM), Universiti Teknologi MARA (UiTM), Jalan Hospital, Sungai Buloh, Selangor, Malaysia ; 4Department of Public Health Medicine, Faculty of Medicine, Universiti Teknologi MARA (UiTM), Jalan Hospital, Sungai Buloh, Selangor, Malaysia; 5Institute of Medical Molecular Biotechnology, Faculty of Medicine (IMMB), Sungai Buloh Campus, Universiti Teknologi MARA (UiTM), Jalan Hospital, Sungai Buloh, Selangor, Malaysia; 6Department of Biochemistry and Molecular Medicine, Faculty of Medicine, Sungai Buloh Campus, Universiti Teknologi MARA (UiTM), Jalan Hospital, Sungai Buloh, Selangor, Malaysia; 7Department of Pathology, Faculty of Medicine, Universiti Teknologi MARA (UiTM), Jalan Hospital, Sungai Buloh, Selangor, Malaysia

Introduction: Despite optimization of risk factors for cardiovascular diseases (CVD) in primary care, deaths due to CVD remains high in Malaysia. Atherosclerosis is a disease of chronic inflammation. This study aimed to determine the concentration of high-sensitivity C-reactive protein (hsCRP) and Interleukin-6 (IL-6) and their associated factors among Malaysian adults with MetS in a primary care clinic.

Methods: A cross sectional study was conducted between July 2020 and February 2021 in an institutional primary care clinic. Adults between 18 to 70 years who fulfilled the MetS criteria based on Joint Interim Statement 2009 
were recruited. Those who were not Malaysian, pregnant, diagnosed of CVD or inflammatory disorders, had limited lifespan or were unable to give informed consent were excluded. Socio-demographics, clinical, anthropometric, fasting serum lipid, fasting blood sugar, glycosylated haemoglobin, hsCRP and IL-6 were measured. Multiple linear regression was performed to identify independent factors associated with hsCRP and IL-6.

Results: The final analysis included 130 participants. The median age was $60.0(\mathrm{IQR}=11)$ years with more females (55.4\%) and Malays (84.6\%). The median hsCRP was $2.3(\mathrm{IQR}=2.5) \mathrm{mg} / \mathrm{L}$. Median IL-6 was $23.5 \quad(\mathrm{IQR}=8.9)$ $\mathrm{pg} / \mathrm{ml}$. After multivariable adjustment, factors associated with hsCRP were BMI ( $\beta=0.020$ [95\% CI: 0.007, 0.031], $\mathrm{P}=0.002)$ and LDL-C $(\beta=0.085$ [95\% CI: 0.029, 0.140], $\mathrm{P}=0.006)$. Factors associated with IL-6 were Malay ethnicity $(\beta=0.124$ [95\% CI: $0.042,0.206], P=0.003)$ and secondary education level or below ( $\beta=0.086$ [95\% CI: $-0.027,0.145]$, $\mathrm{P}=0.005)$.

Conclusion: The concentration of hsCRP and IL-6 remains comparable with other studies. BMI and LDL-C were significantly associated with hsCRP. Malay ethnicity and secondary education level or below were associated with IL-6. This study underscores the importance of these risk factors reduction among high-risk patients in primary care.

\section{ACKNOWLEDGMENT}

The authors would like to thank the Director of Health Malaysia for permission to present their studies at the 23rd Family Medicine Scientific Conference 2021 and publish their abstracts. 
